\title{
CLIMATIC DATA FOR MIRROR LAKE, WEST THORNTON, NEW HAMPSHIRE 1986
}

By

A.M. Sturrock, D.C. Buso, JL. Scarborough, T.C. Winter

\section{U.S. GEOLOGICAL SURVEY}

Open-File Report 88-494

Lakewood, Colorado 1988 
DEPARTMENT OF THE INTERIOR

DONALD PAUL HODEL, Secretary

U. S. GEOLOGICAL SURVEY

Dallas L. Peck, Director

For additional information write to:

Thomas C. Winter

U.S. Geological Survey

Box 25046, MS 413

Denver Federal Center

Denver, CO 80225
Copies of this report can be purchased from:

U.S. Geological Survey Books and Open-File Reports Section Denver Federal Center, Bldg. 810 Box 25425

Denver, CO 80225 
Abstract-1.2.

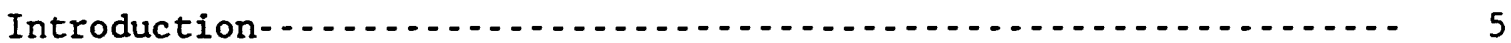

Data collection and presentation-1... 5

Acknowledgments

References $\ldots \ldots$

\section{TABLES}

Table 1. Summary of 1986 energy-budget data................. 8

2. Summary of 1986 temperature data at the raft station-.-. 39

3. Summary of 1986 wind-speed data at the raft station-... 46

4. Summary of the 1986 radiation data at the land station-- 61 
METRIC CONVERSION FACTORS

$\begin{array}{lll}\text { Multiply } & \text { By } & \text { To obtain } \\ \text { centimeter } & 0.394 & \text { inch } \\ \text { meter } & 3.281 & \text { foot } \\ \text { kilometer } & 0.621 & \text { mile } \\ \text { millibar } & 0.0145 & \text { pound per square inch } \\ \text { millibar } & 1.0197 & \text { gram per square centimeter } \\ \text { mile per hour } & 1.609 & \text { kilometer per hour } \\ \begin{array}{l}\text { calories per square centimeter } \\ \text { per minute }\end{array} & 697.3 & \text { watt per square meter }\end{array}$

To convert degrees Celsius $\left({ }^{\circ} \mathrm{C}\right)$ to degrees Fahrenheit $\left({ }^{\circ} \mathrm{F}\right)$ use the following formula: $\left({ }^{\circ} \mathrm{C} \times 9 / 5\right)+32={ }^{\circ} \mathrm{F}$. 
CLIMATIC DATA FOR MIRROR LAKE, WEST THORNTON, NEW HAMPSHIRE, 1986

By A.M. Sturrock, D.C. Buso, J.L. Scarborough, and T.C. Winter

\begin{abstract}
Research on the hydrology of Mirror Lake, West Thornton, New Hampshire, includes study of evaporation. Presented here are those climatic data needed for energy-budget and mass-transfer evaporation studies, including: water-surface temperature, dry-bulb and wet-bulb air temperatures, vapor pressure at and above the water surface, wind speed, and short-and longwave radiation. Data are collected at raft and land stations.
\end{abstract}

\title{
INTRODUCTION
}

Climatic data are being collected at Mirror Lake, West Thornton, New Hampshire, as part of a continuing study of the hydrology of the lake by the U.S. Geological Survey, Cornell University, and the New York Botanical Garden, Institute of Ecosystem Studies. Mirror Lake is one of several lakes in different parts of the United States that have been selected for intensive study of hydrological and related chemical and biological processes. The rationale for selection of Mirror Lake is given by Winter (1984). Climatic data for the 1981 and 1982 open-water seasons are given in Sturrock and others (1984), those for the 1984 open-water season are given in Sturrock and others (1986), and those for the 1985 open-water season are given in Sturrock and others (1988).

\section{DATA COLLECTION AND PRESENTATION}

Data presented here are being collected principally for studies of evaporation; therefore, data are collected only during the time the lake is ice-free. Data for 1986 were collected from April 17 (Julian day 107) to November 21 (Julian day 325). Within table 1, the data are grouped according to energy-budget periods; the periods are defined by the dates thermal surveys were made in the lake. For example, the first energy-budget period for 1986 is Julian days 107 through 113.

Climatic instruments are located on a raft near the middle of the lake and at a land station about 0.4 kilometer west of the lake. Instruments on the raft include anemometers at 1,2 , and 3 meters above the water surface, a thermistor psychrometer with dry- and wet-bulb temperature sensors fixed at 2 meters above the water surface, and a water-temperature sensor located beneath the raft at a depth of about 1 centimeter. Data from the above sensors are recorded by a digital data logger located on the raft. The data logger scans the sensors every minute and calculates hourly and daily 
averages. In addition, maximum and minimum values and the times they occur are stored and recorded at midnight of each day for selected sensors. Vapor pressure of water $\left(e_{0}\right)$ is calculated using water-temperature data and assuming the air is completely saturated at the air-water interface. Additional analog instruments for measuring water-surface temperature and wind speed also are located on the raft. These are used to backup the primary instruments for quality control and for filling in missing data. Calibration checks with laboratory thermometers and motorized psychrometers are made weekly.

The land station consists of short-wave and long-wave radiometers, located at the U.S. Forest Service's Hubbard Brook Station. These data also are recorded by a digital-data logger that operates similarly to the one on the raft. A backup hygrothermograph that records air temperature and relative humidity is located on the shore of Mirror Lake.

Data presented here are daily summaries. For periods during which the primary instruments were not operating properly, daily values were obtained by regression using data from backup instruments, provided a satisfactory statistical relation could be established. Data used to establish regressions were selected so they bracketed the period of missing or inadequate data. Only table 1 , which is considered to be the primary source of data for evaporation studies, includes values obtained by regression or some other form of estimation, as indicated in the footnotes to table 1 .

Although only daily values are reported here, hourly values also were recorded. Hourly values are voluminous and expensive to reproduce, but they are available for all or part of the period of record, on request to $T$. $C$. Winter.

\section{ACKNOWLEDGMENTS}

We are grateful to Robert Pierce and Wayne Martin of the U.S. Forest Service for allowing us to place the land station at Hubbard Brook Experimental Forest Headquarters. We also thank Polly Ann Frost for permission to place the hygrothermograph on her property. Partial funding for this study is from a National Science Foundation grant to G. E. Likens (The New York Botanical Garden, Institute of Ecosystem Studies.) and F. H. Bormann (Yale University).

\section{REFERENCES}

Sturrock, A.M., Buso, D.C., Bieber, G.M., Engelbrecht, L.G., and Winter, T.C., 1984, Climatic data for Mirror Lake, West Thornton, New Hampshire, 1981-82: U.S. Geological Survey Open-File Report 84-816, $56 \mathrm{p}$.

Sturrock, A.M., Buso, D.C., Scarborough, J.L., and Winter, T.C., 1986 , Climatic data for Mirror Lake, West Thornton, New Hampshire, 1984: U.S. Geological Survey Open-File Report 86-134, 70 p.

Sturrock, A. M., Buso, D. C., Scarborough, J. L., and Winter, T. C., 1988, Climatic data for Mirror Lake, west Thornton, New Hampshire, 1985: U. S. Geological Survey Open-File Report 87-682, 64 p. 
Winter, T.C., 1984, Geohydrologic setting of Mirror Lake, West Thornton, New Hampshire: U.S. Geological Survey Water-Resources Investigations Report 84-4266, 61 p. 


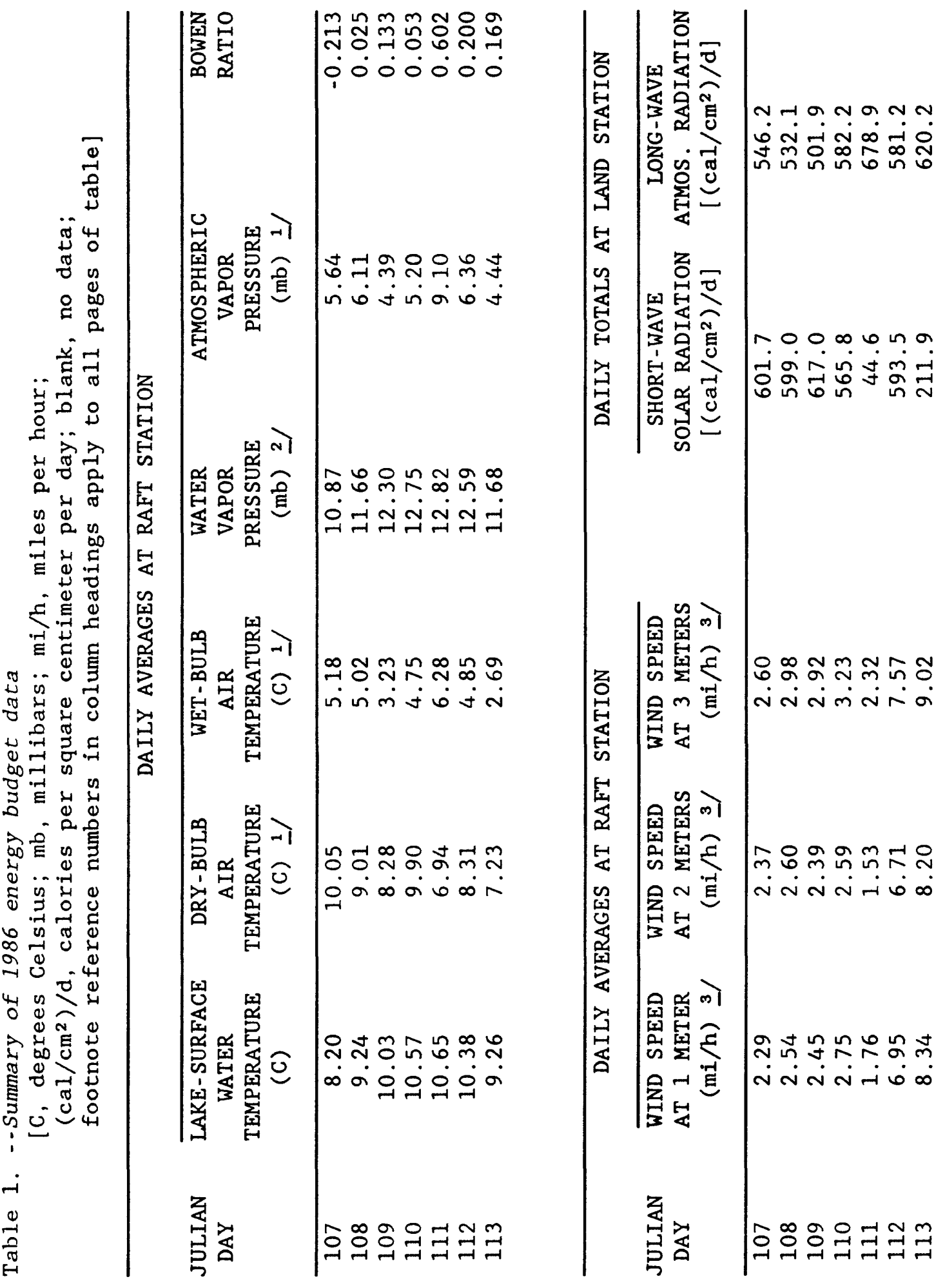




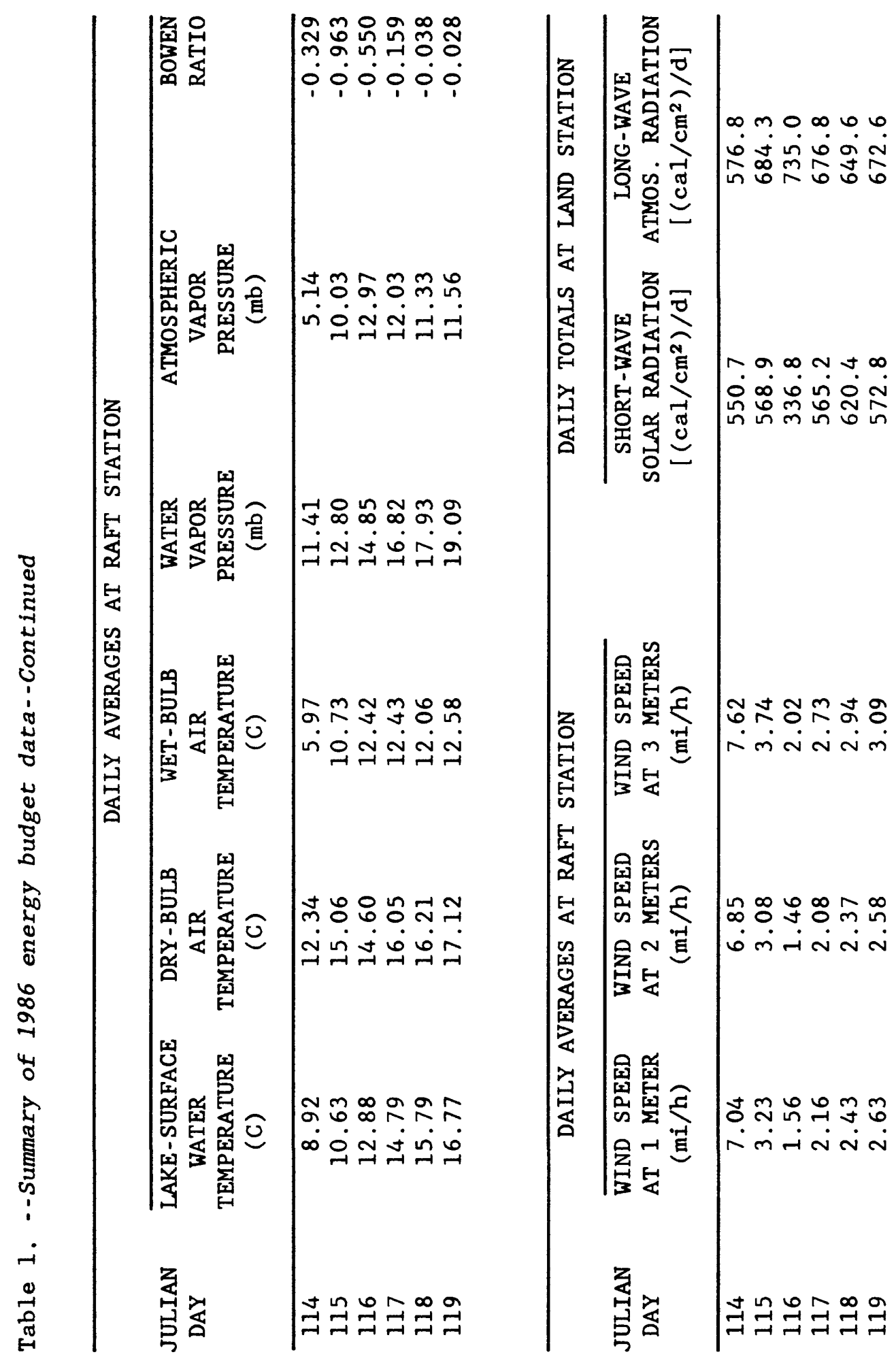




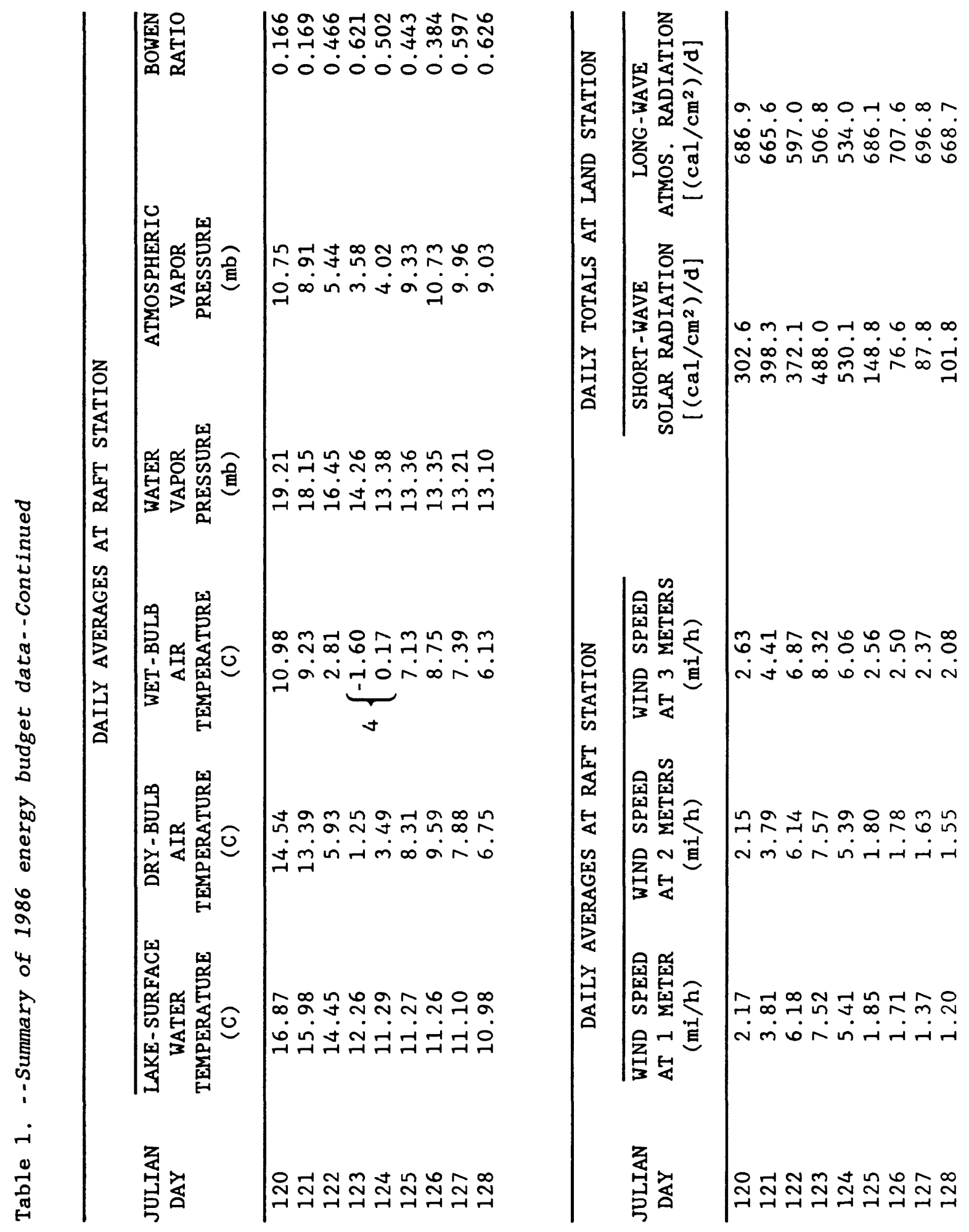




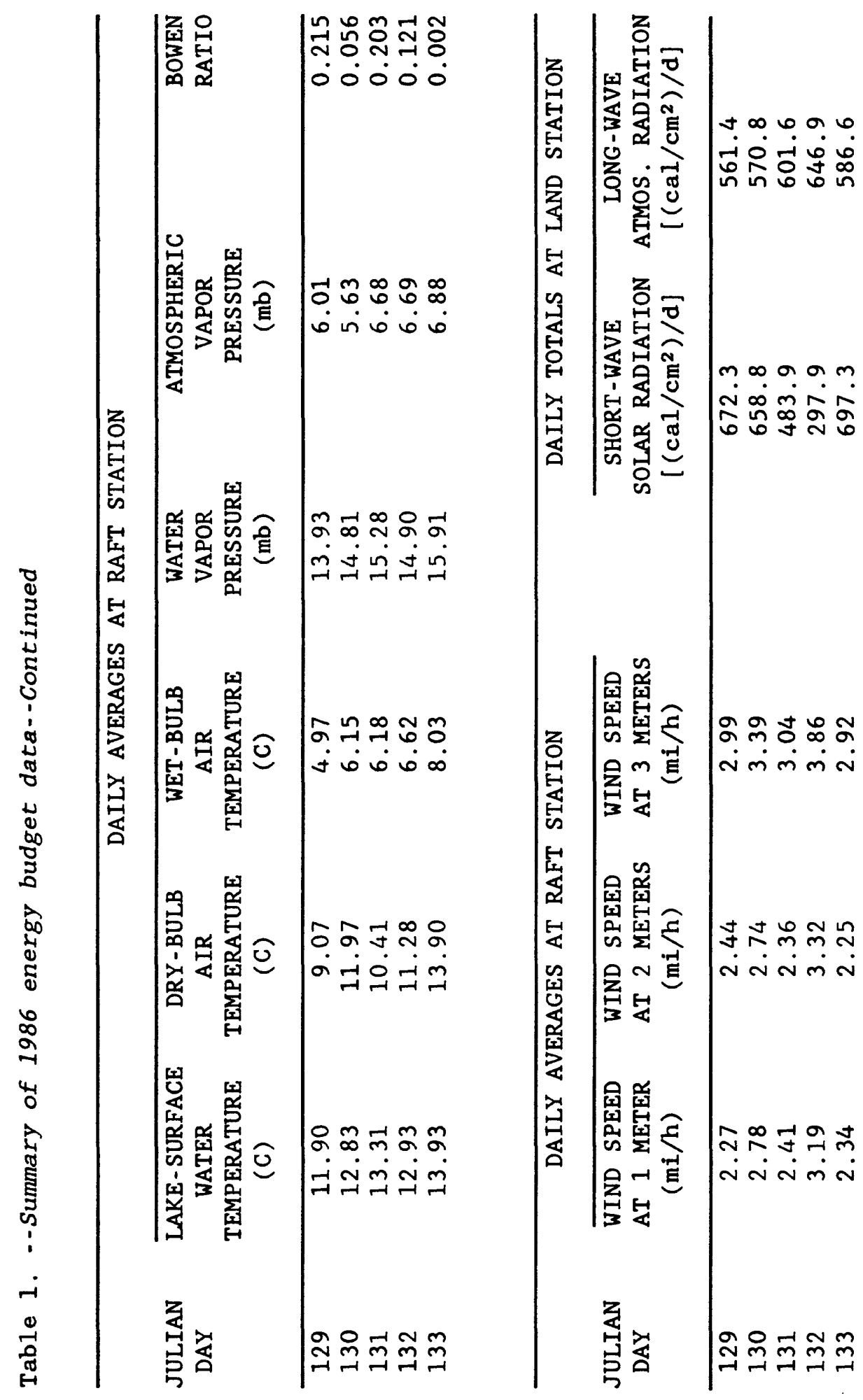




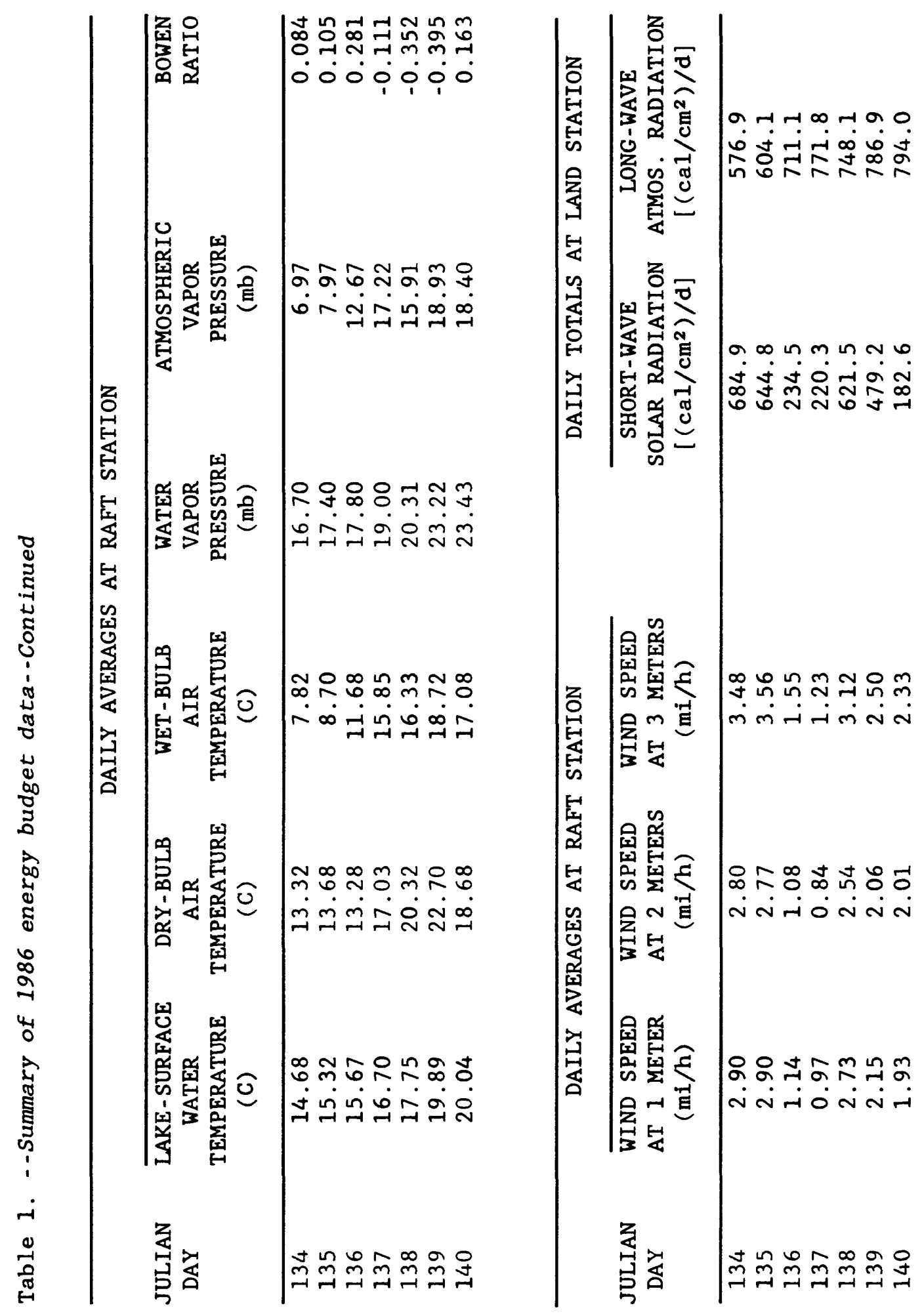




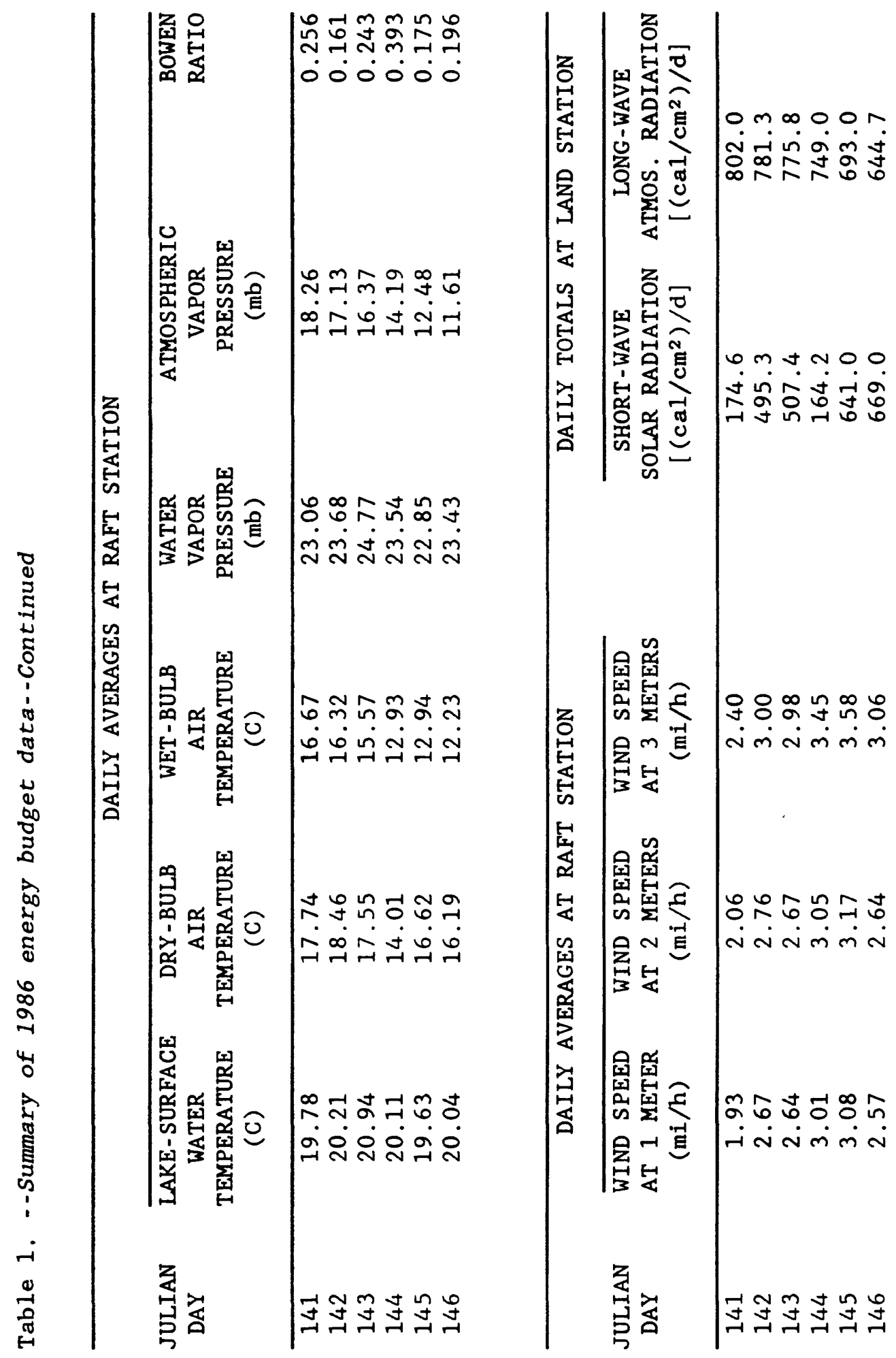




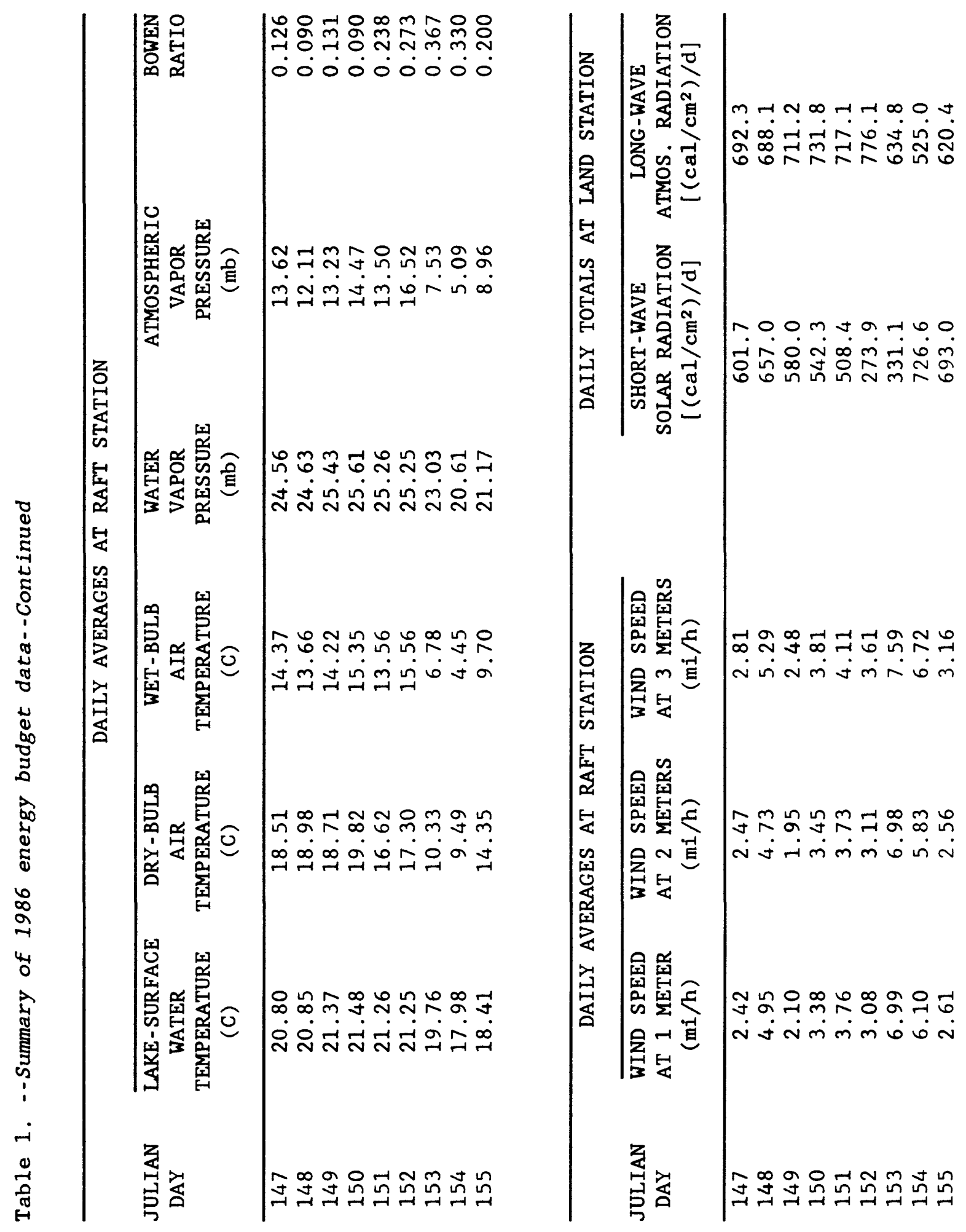




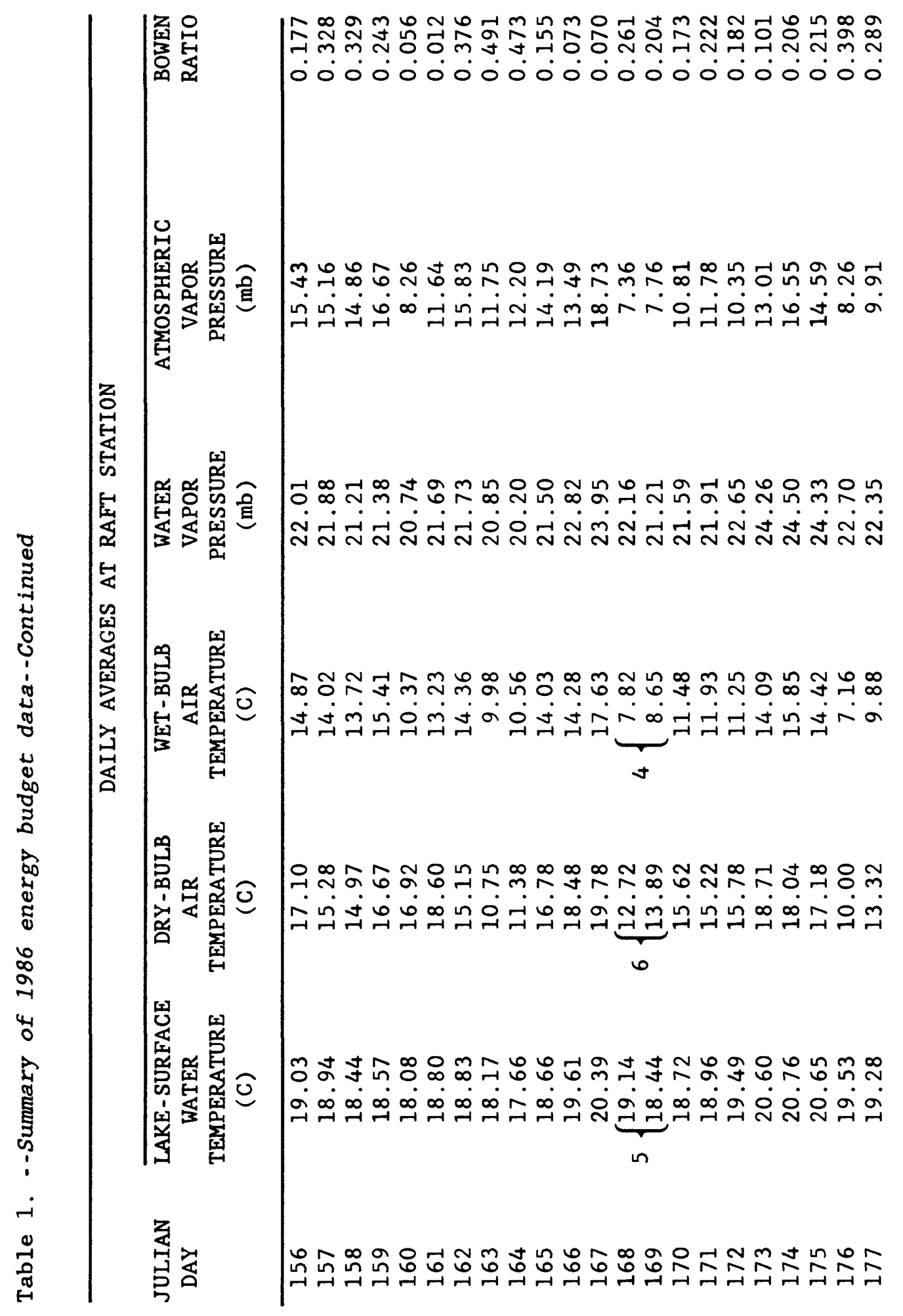




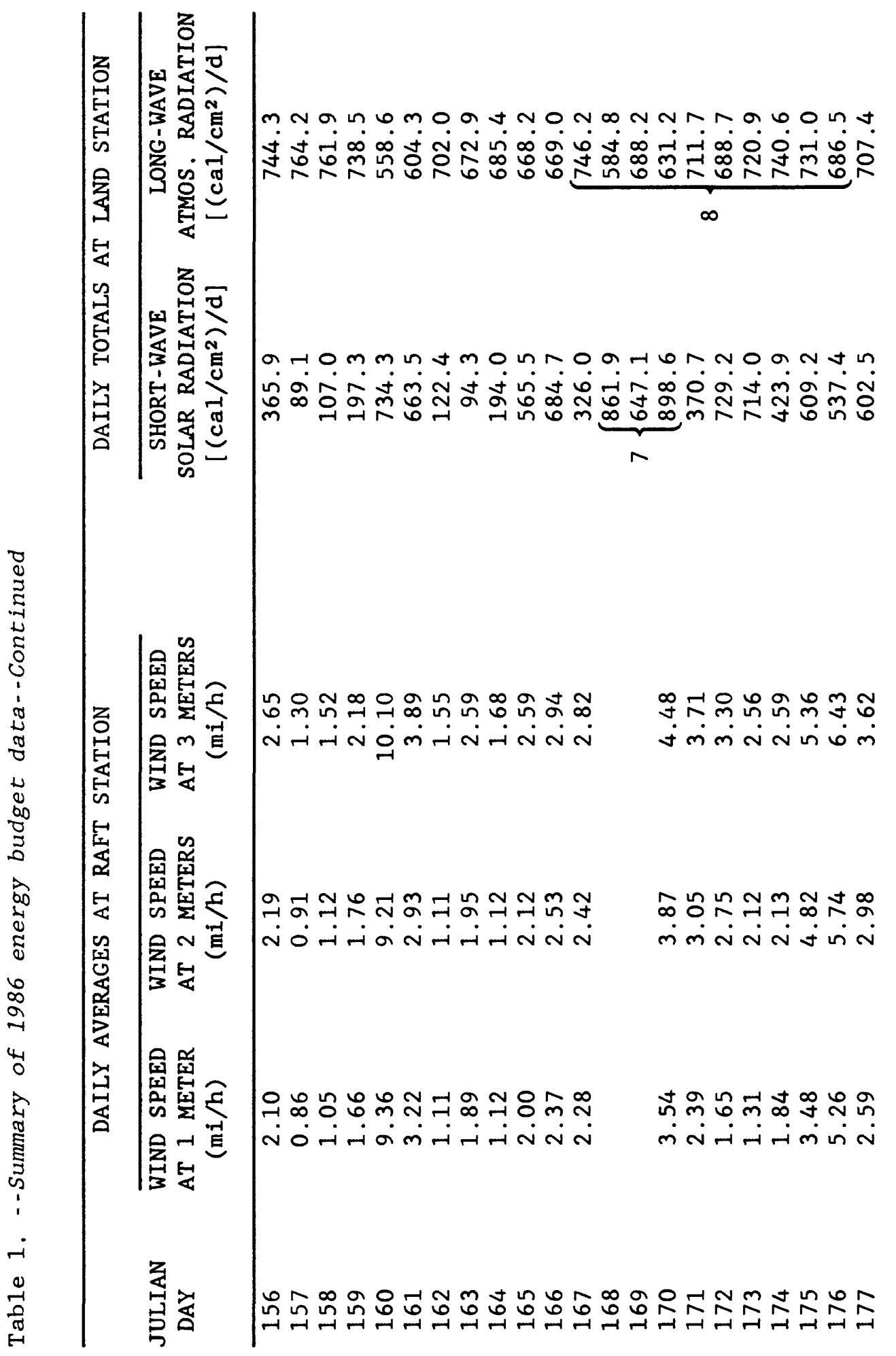




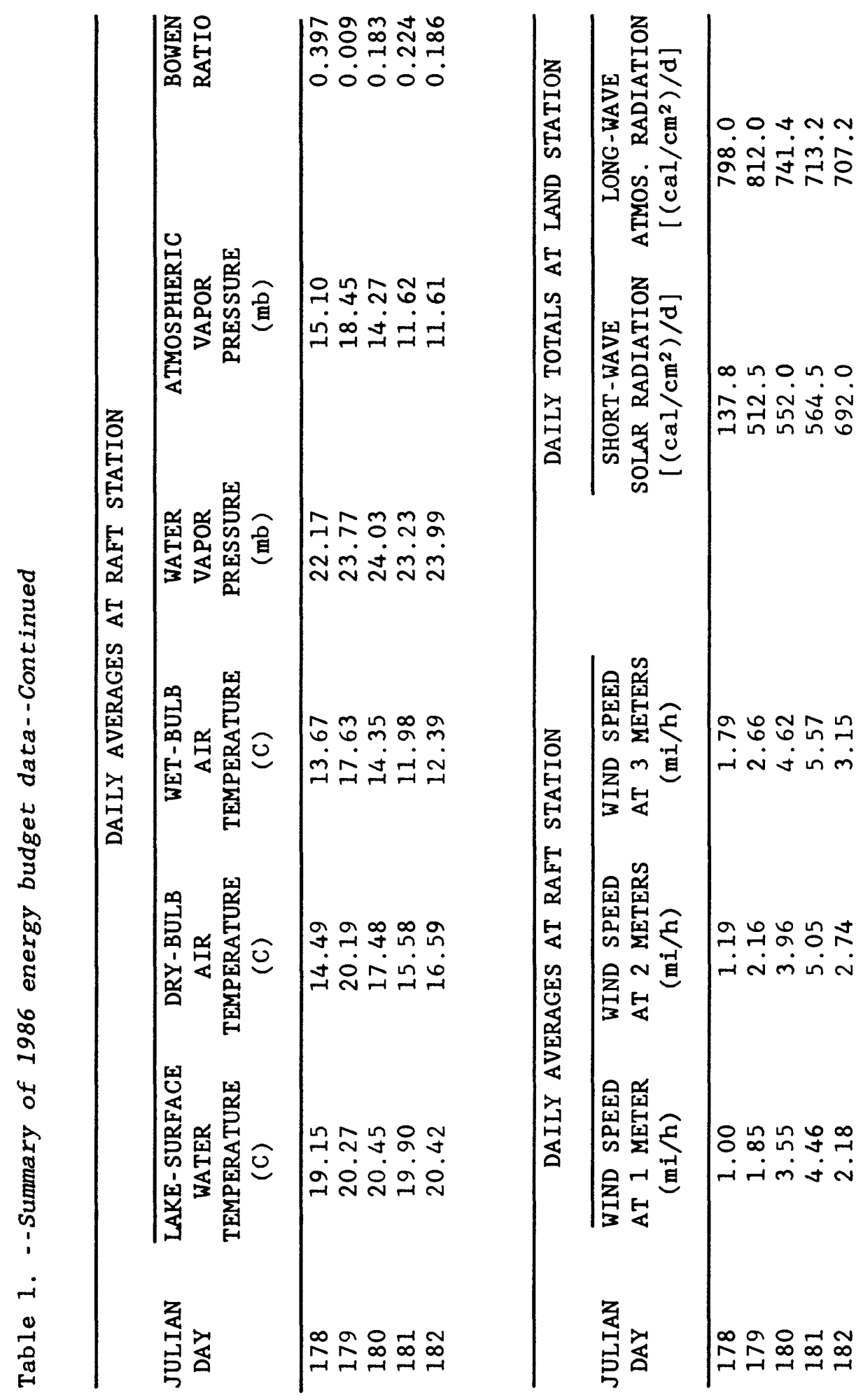




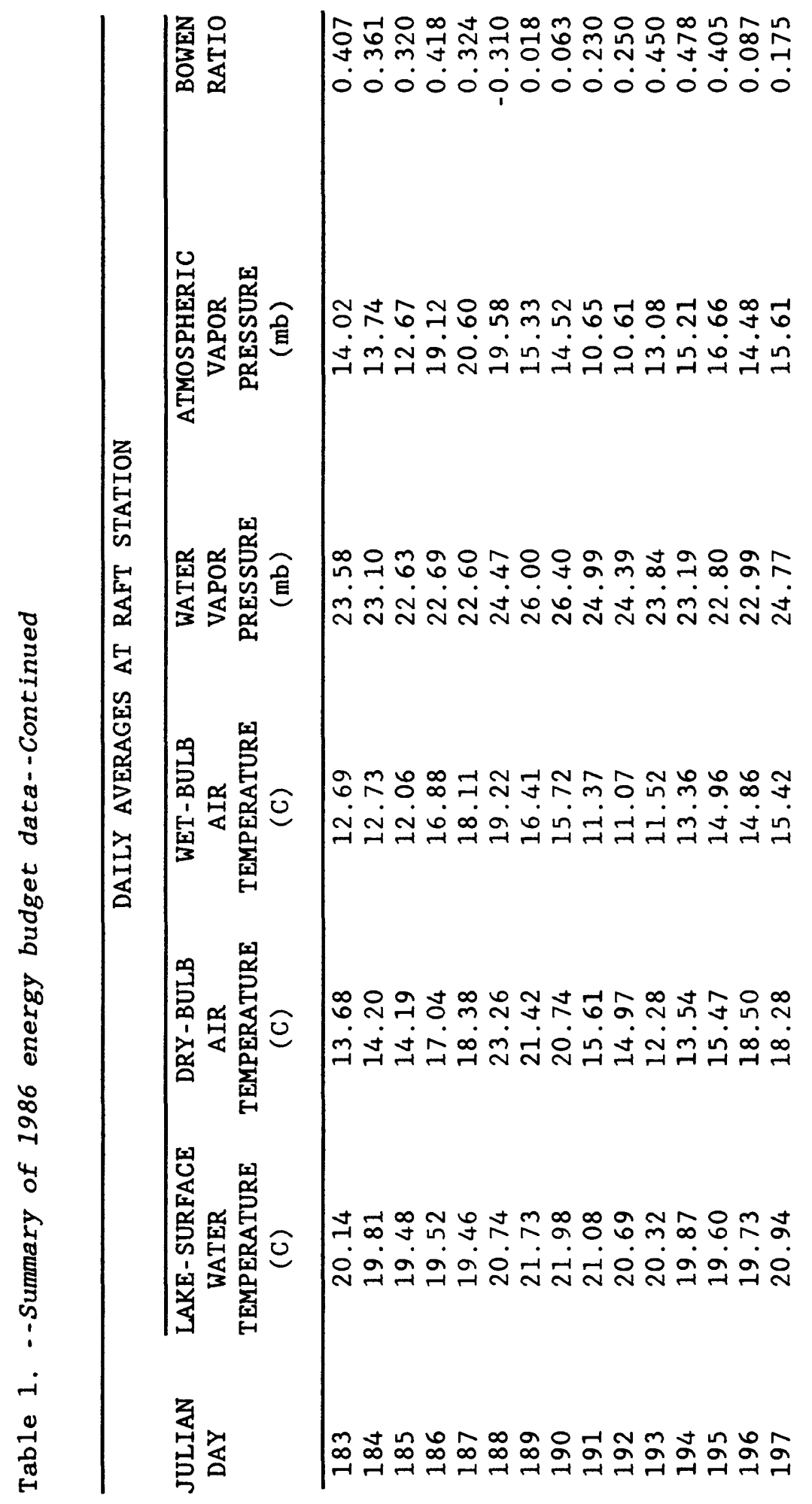




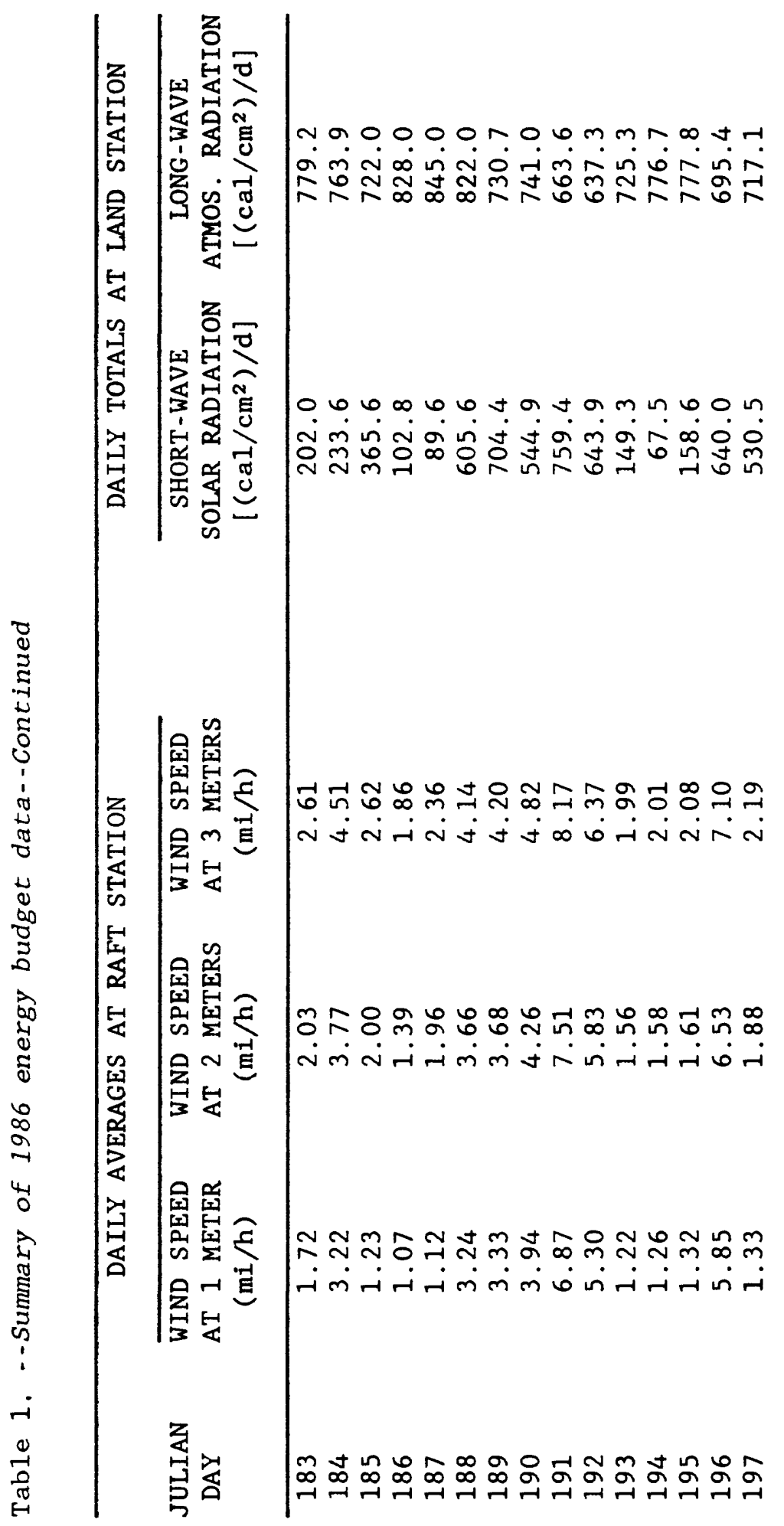




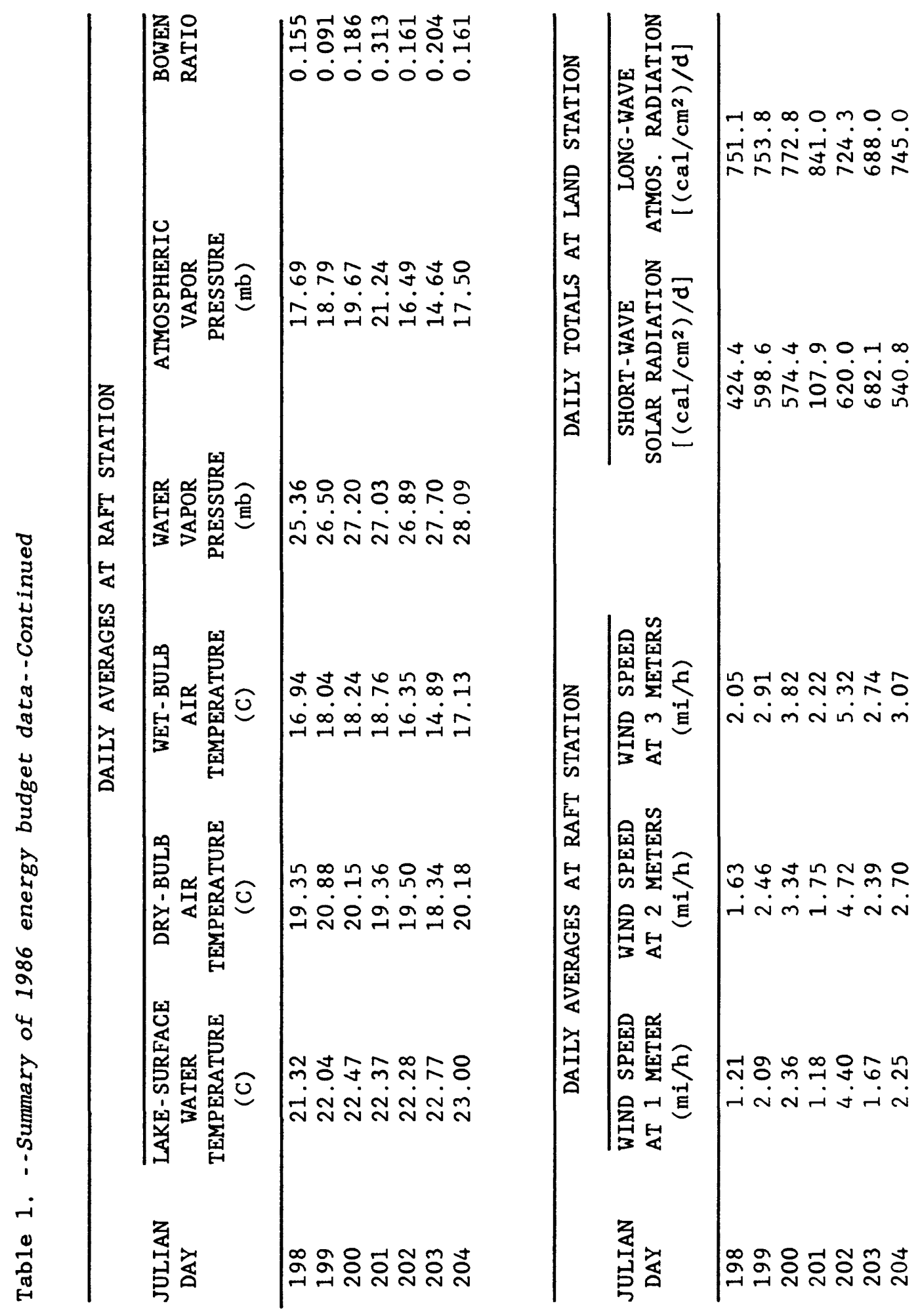




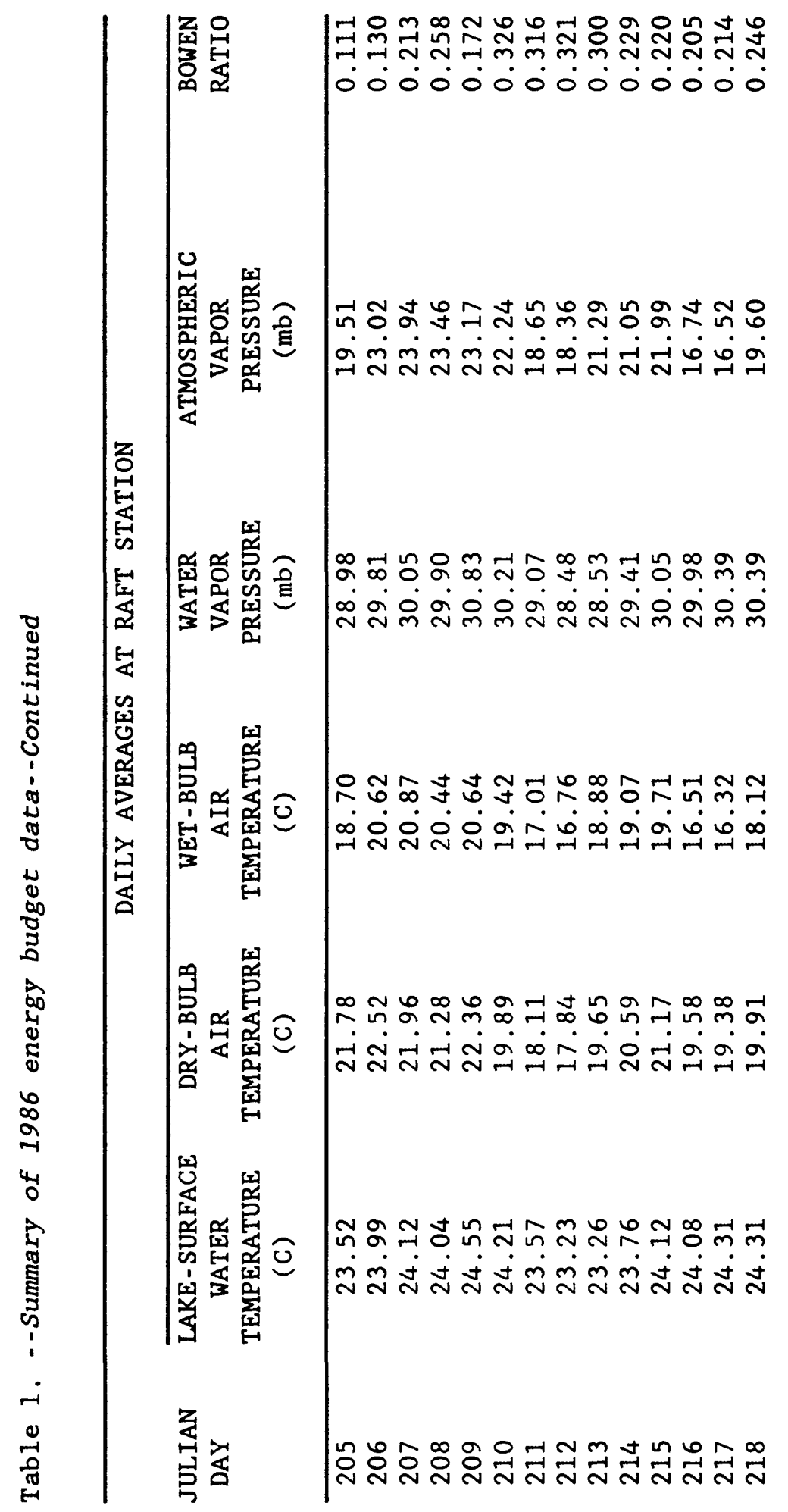




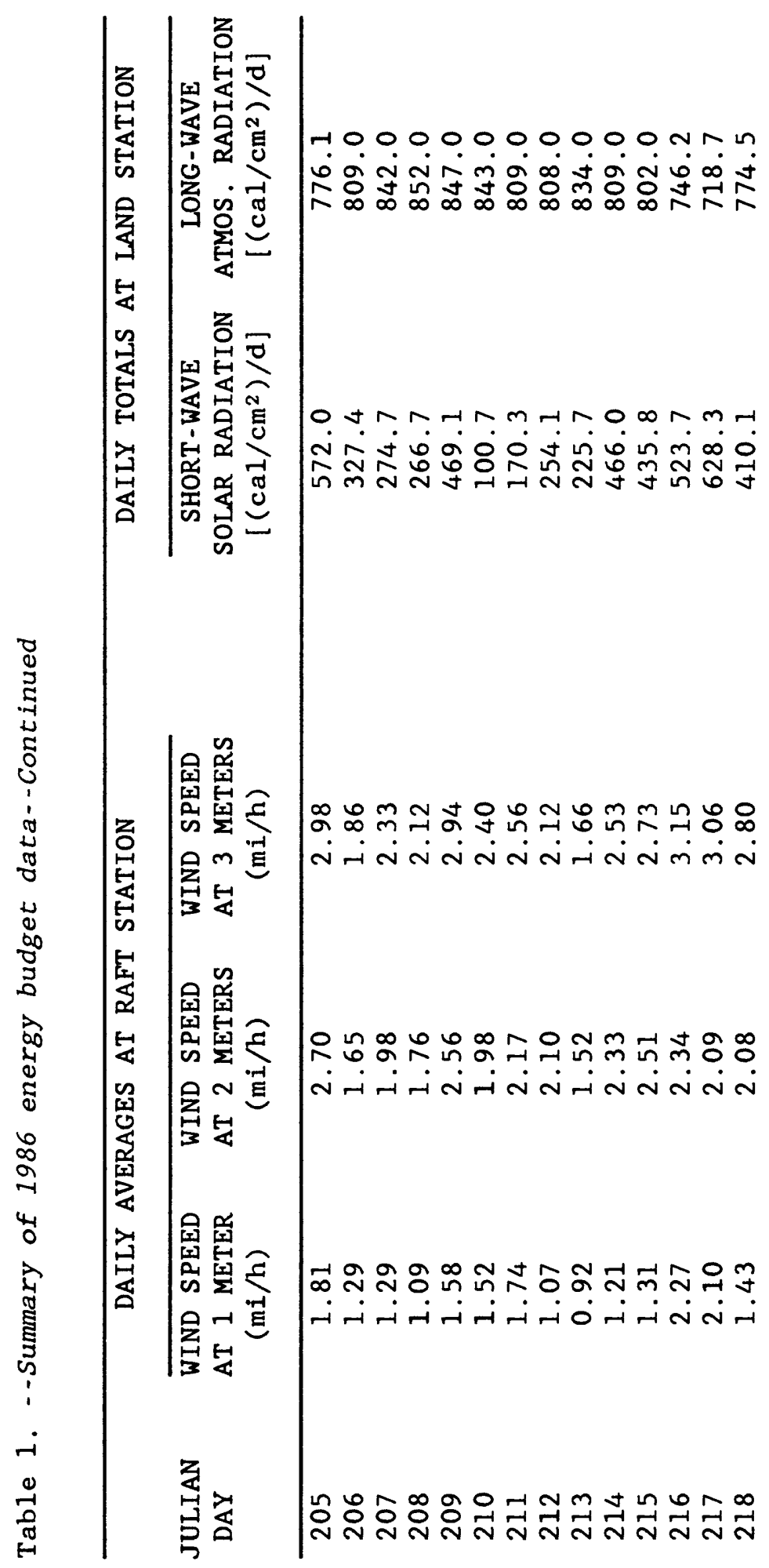




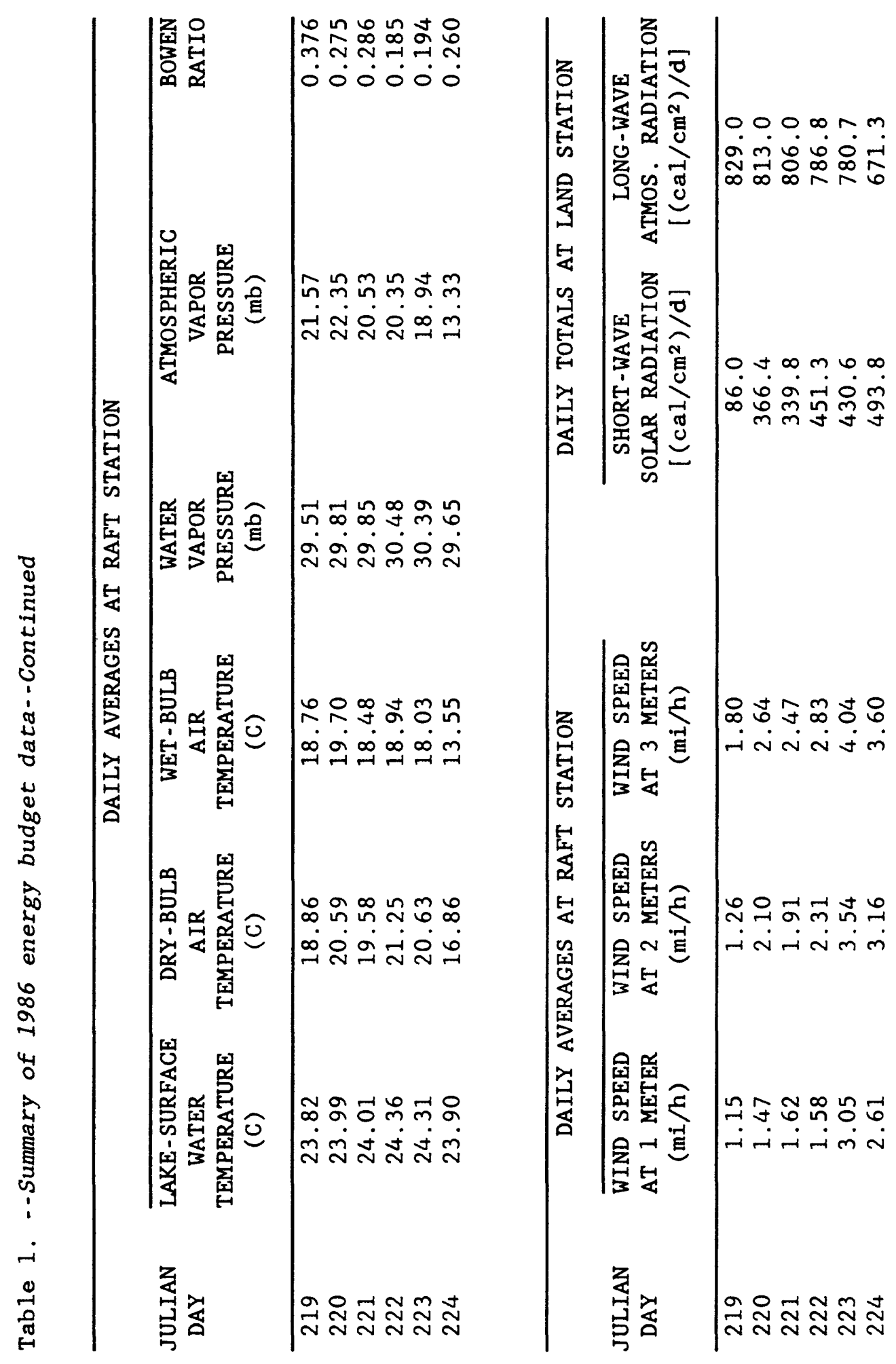




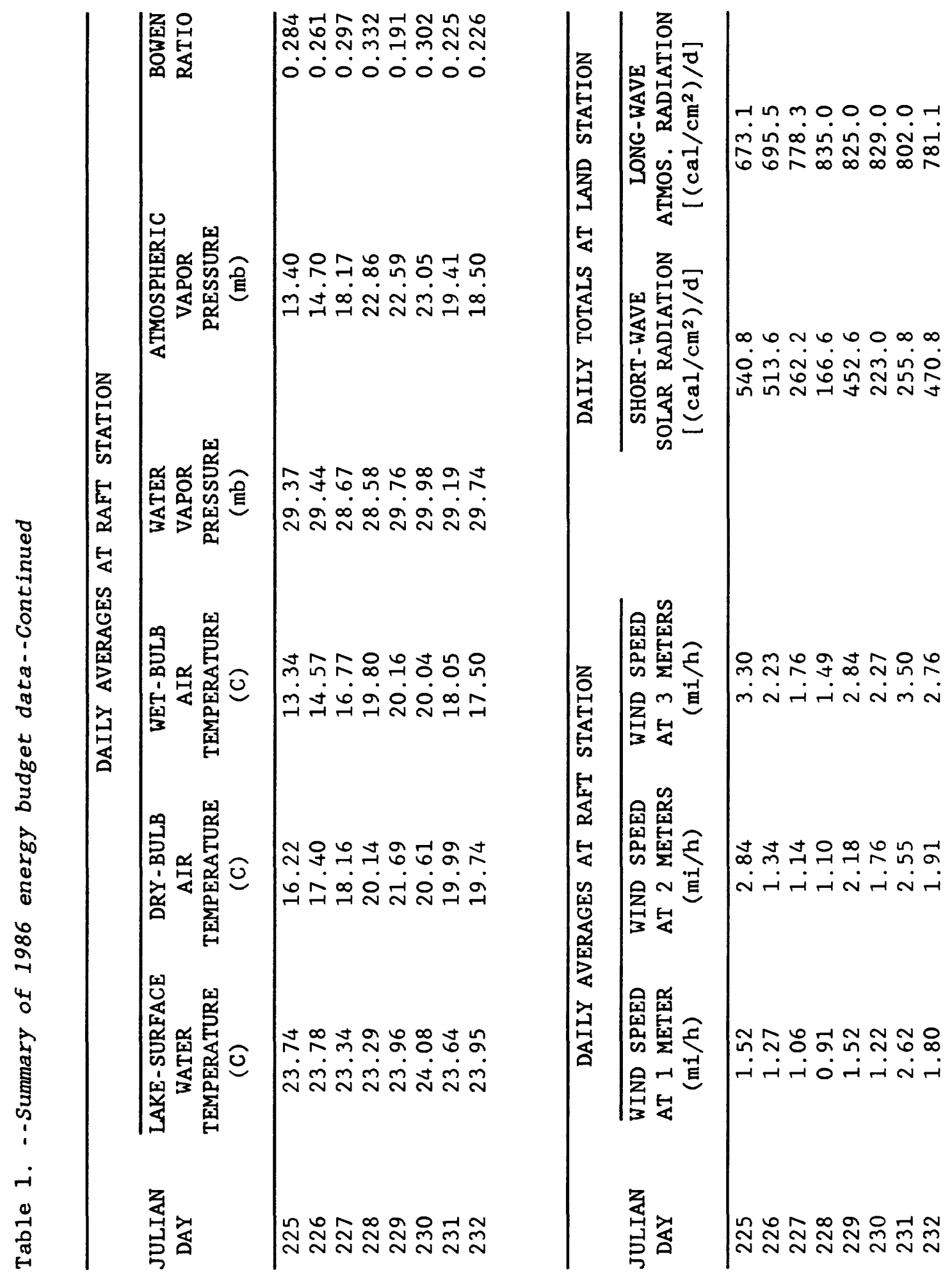




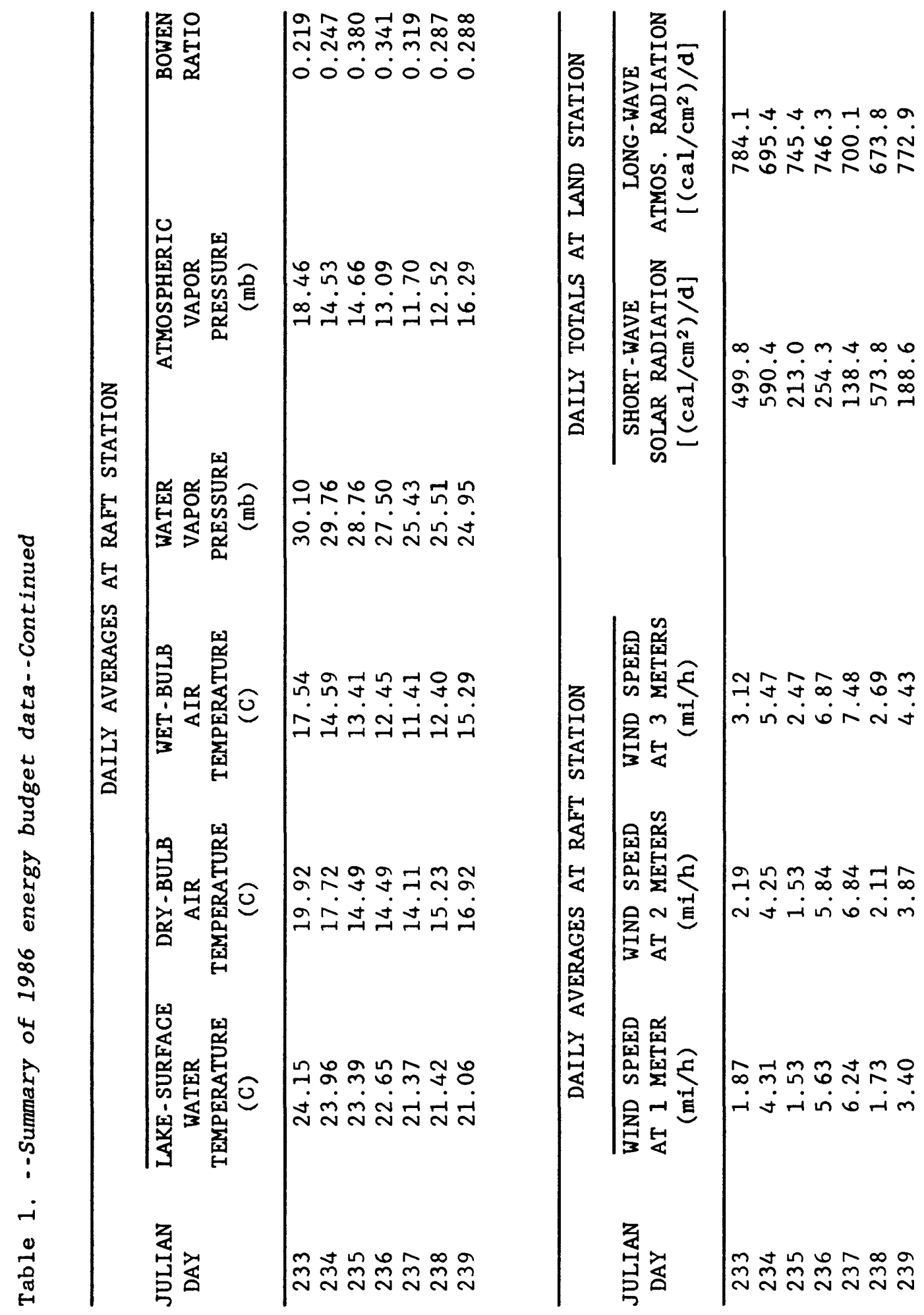




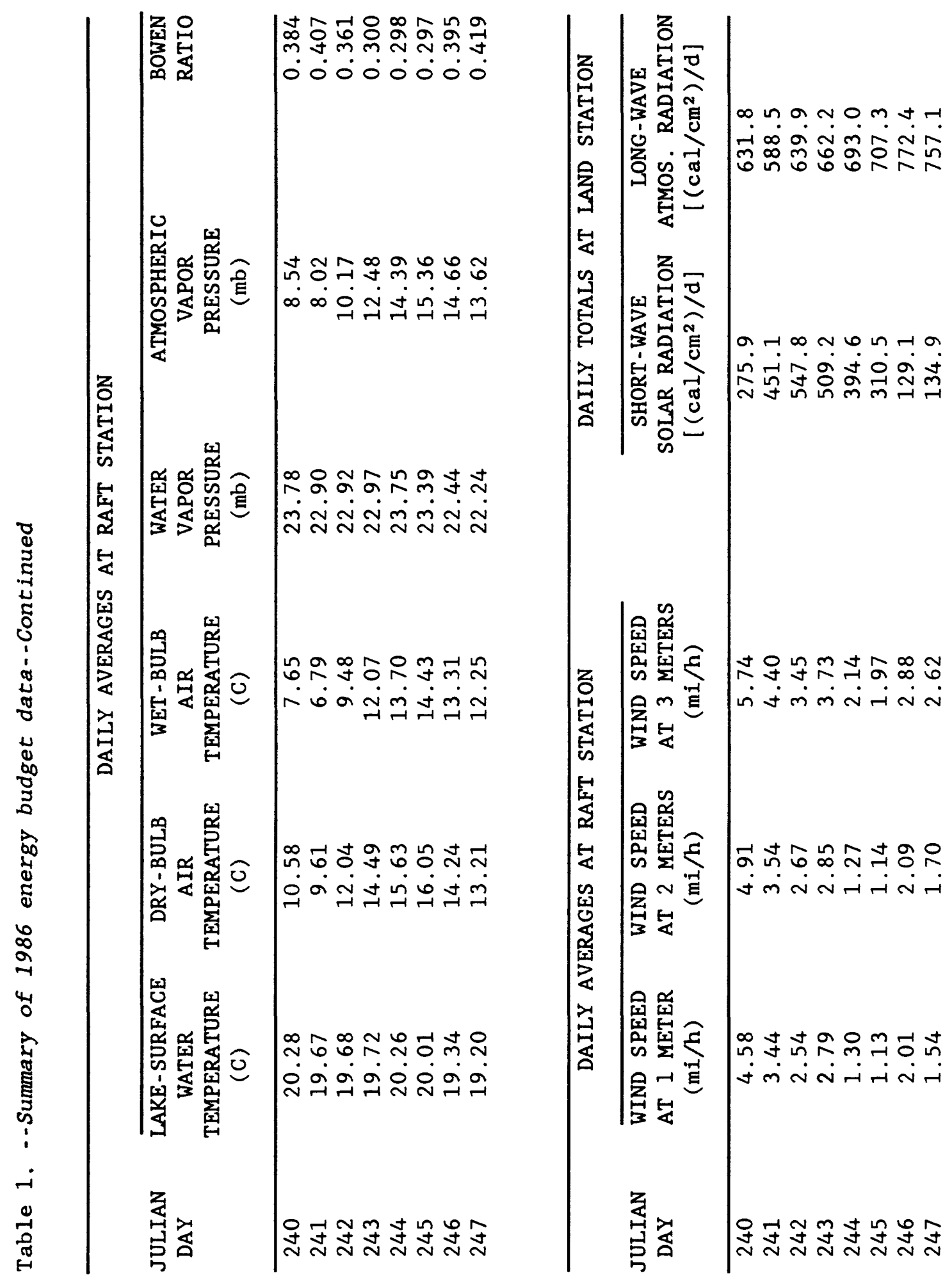




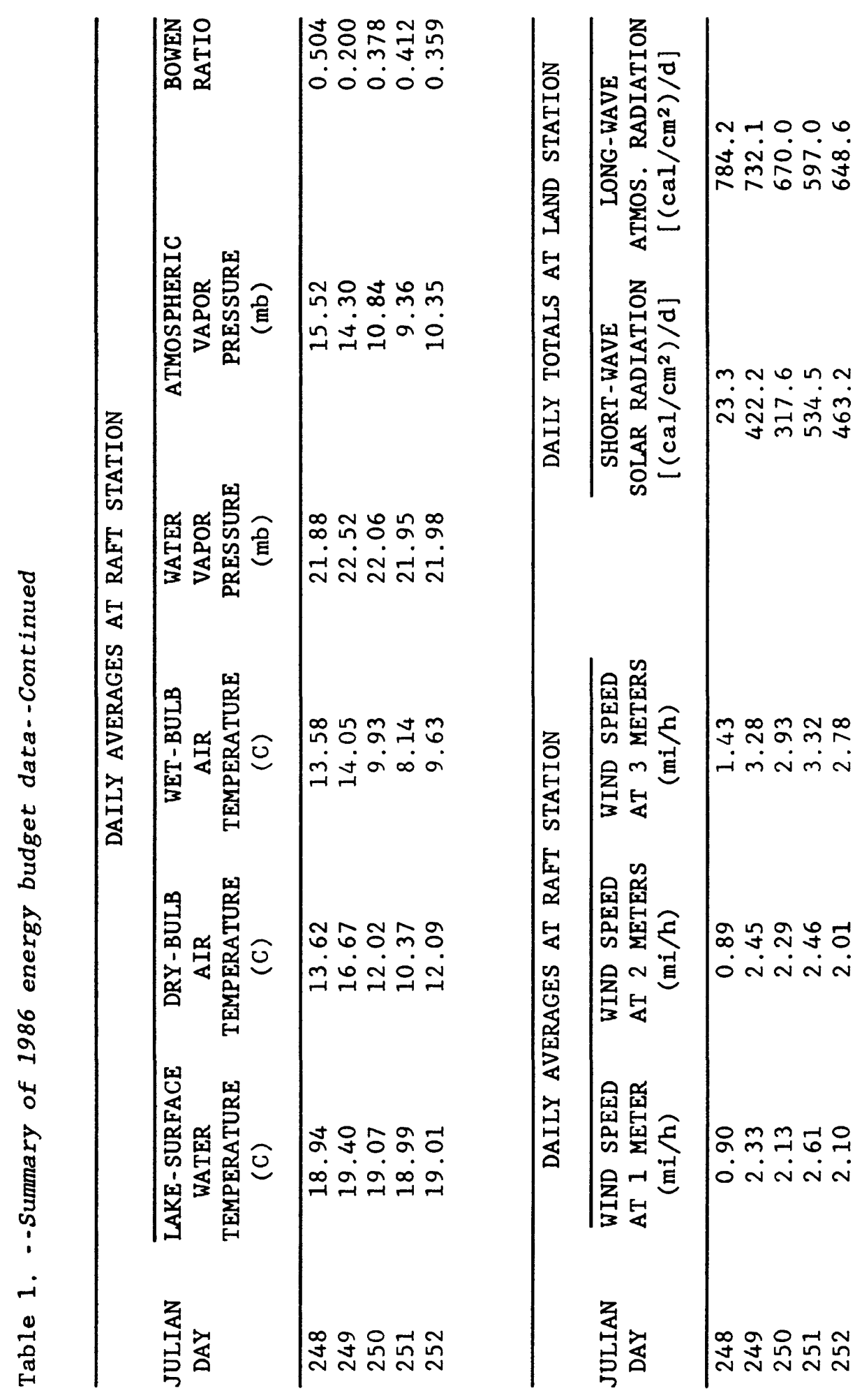




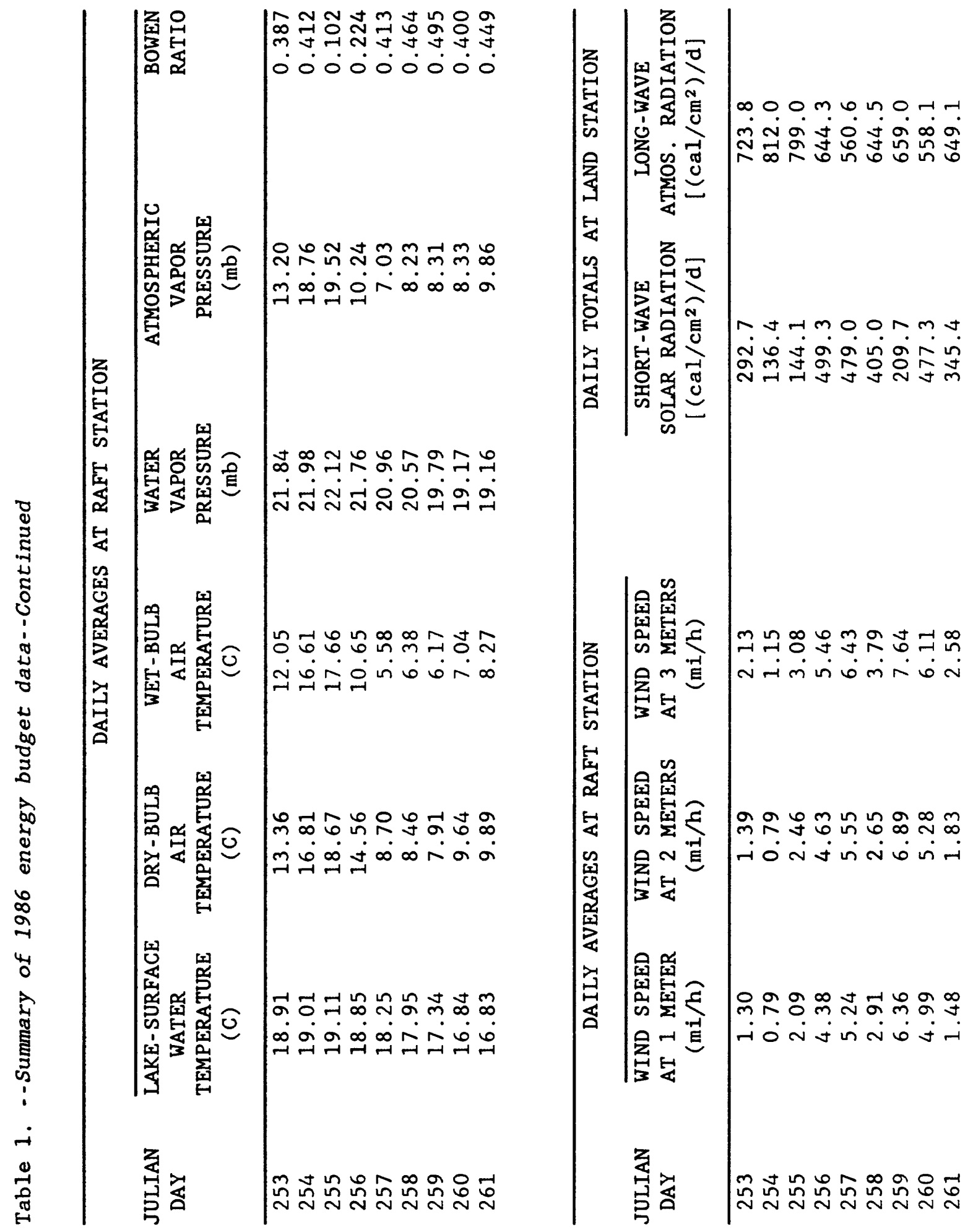




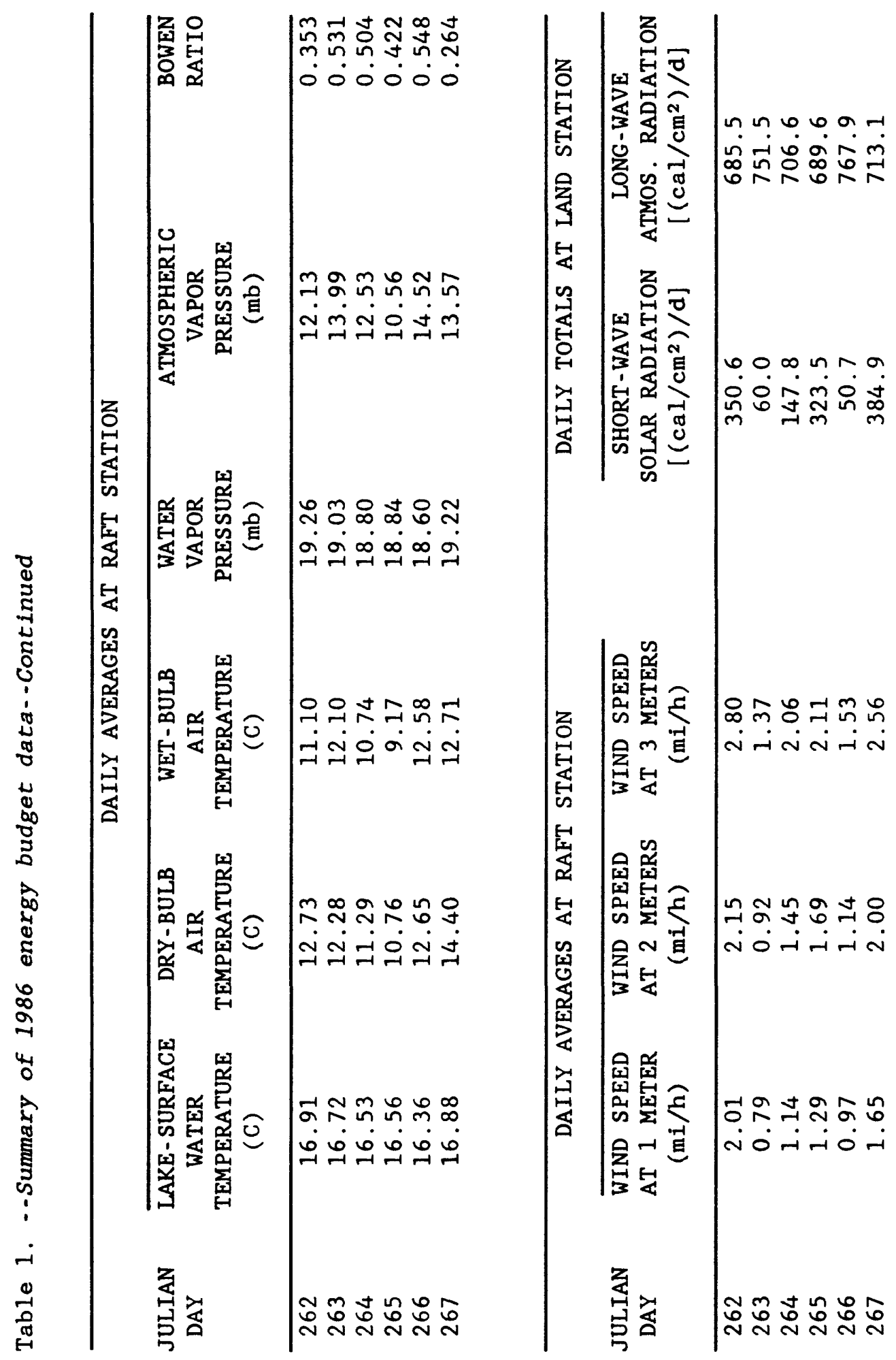




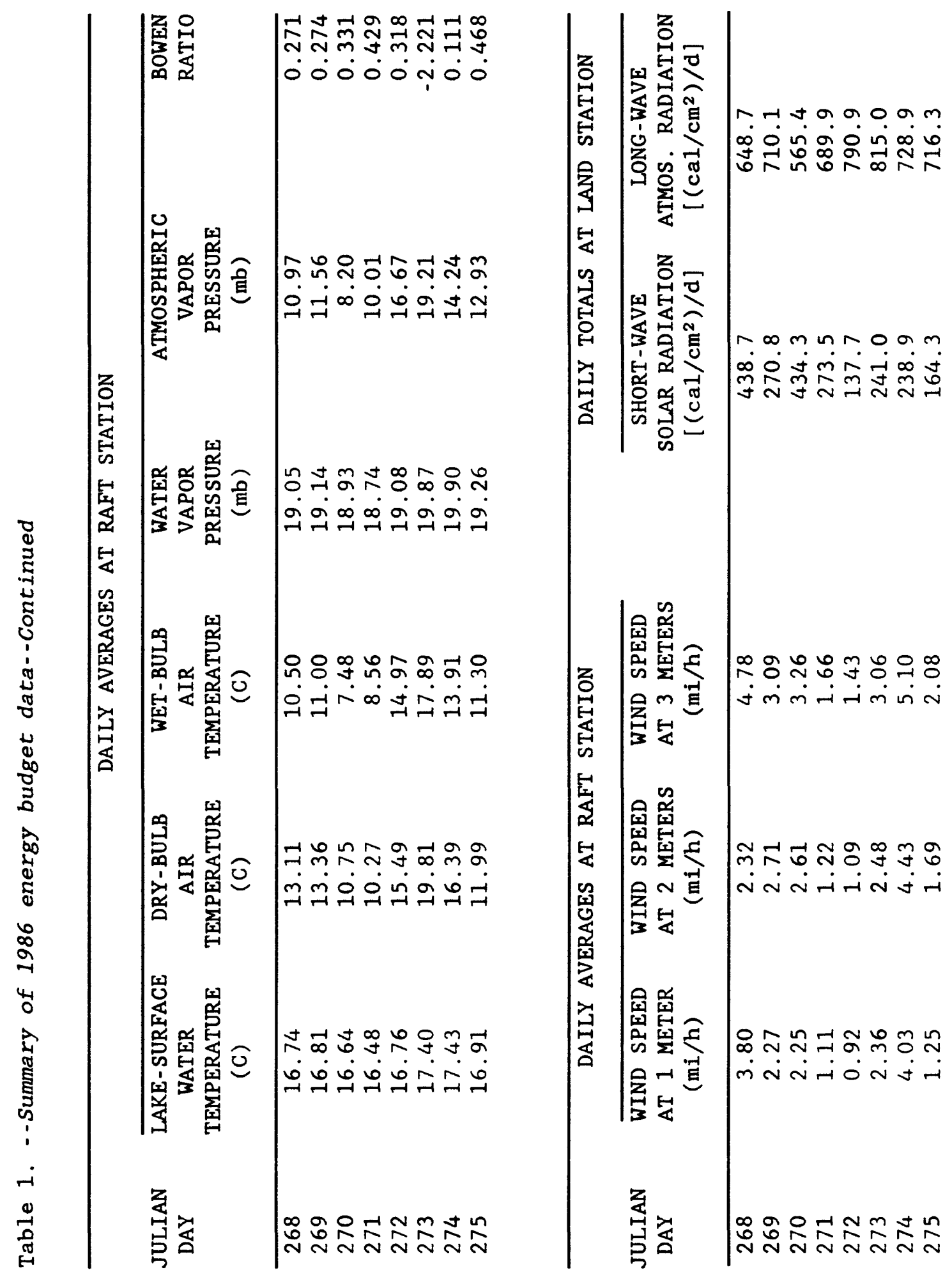




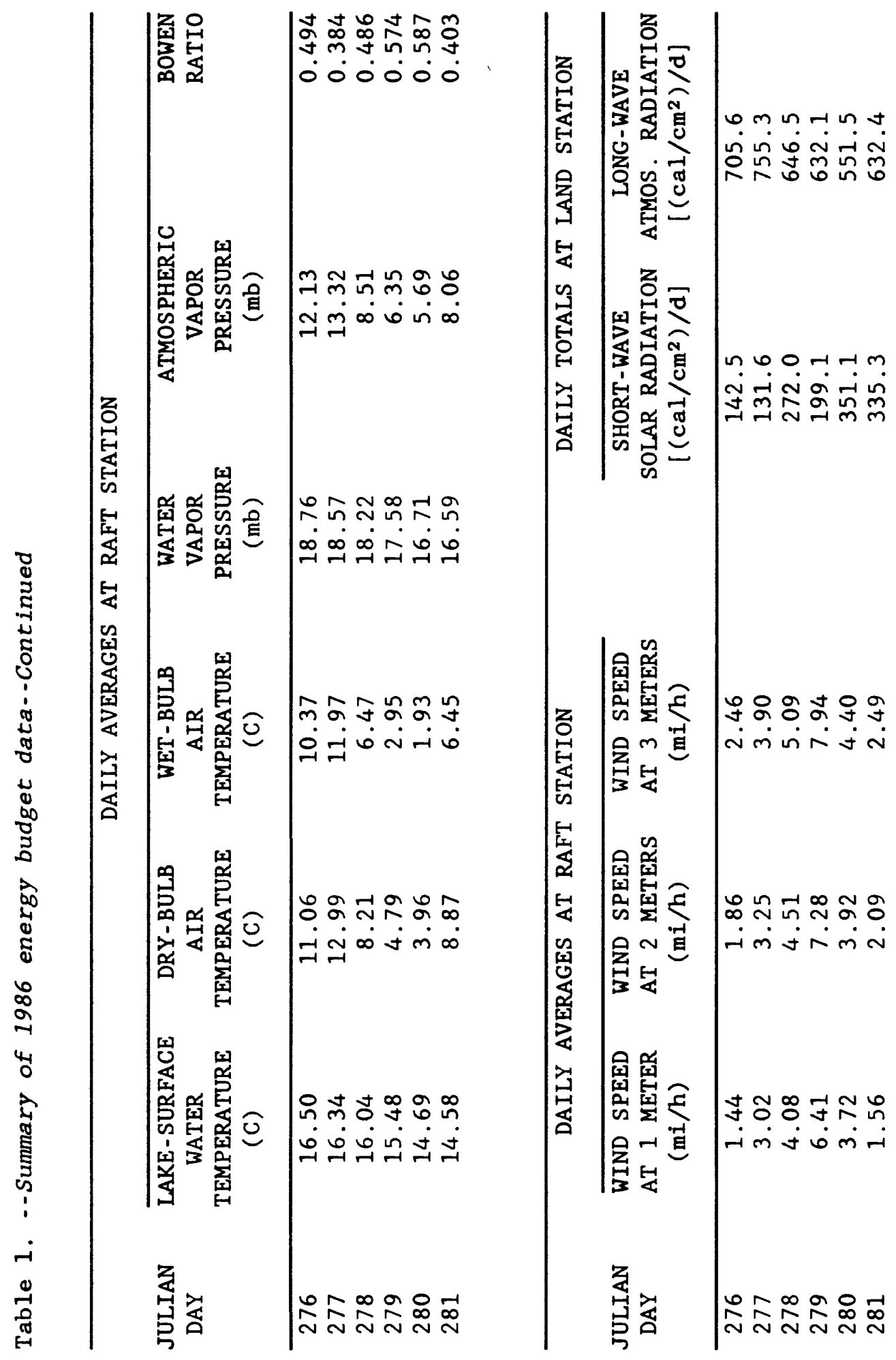




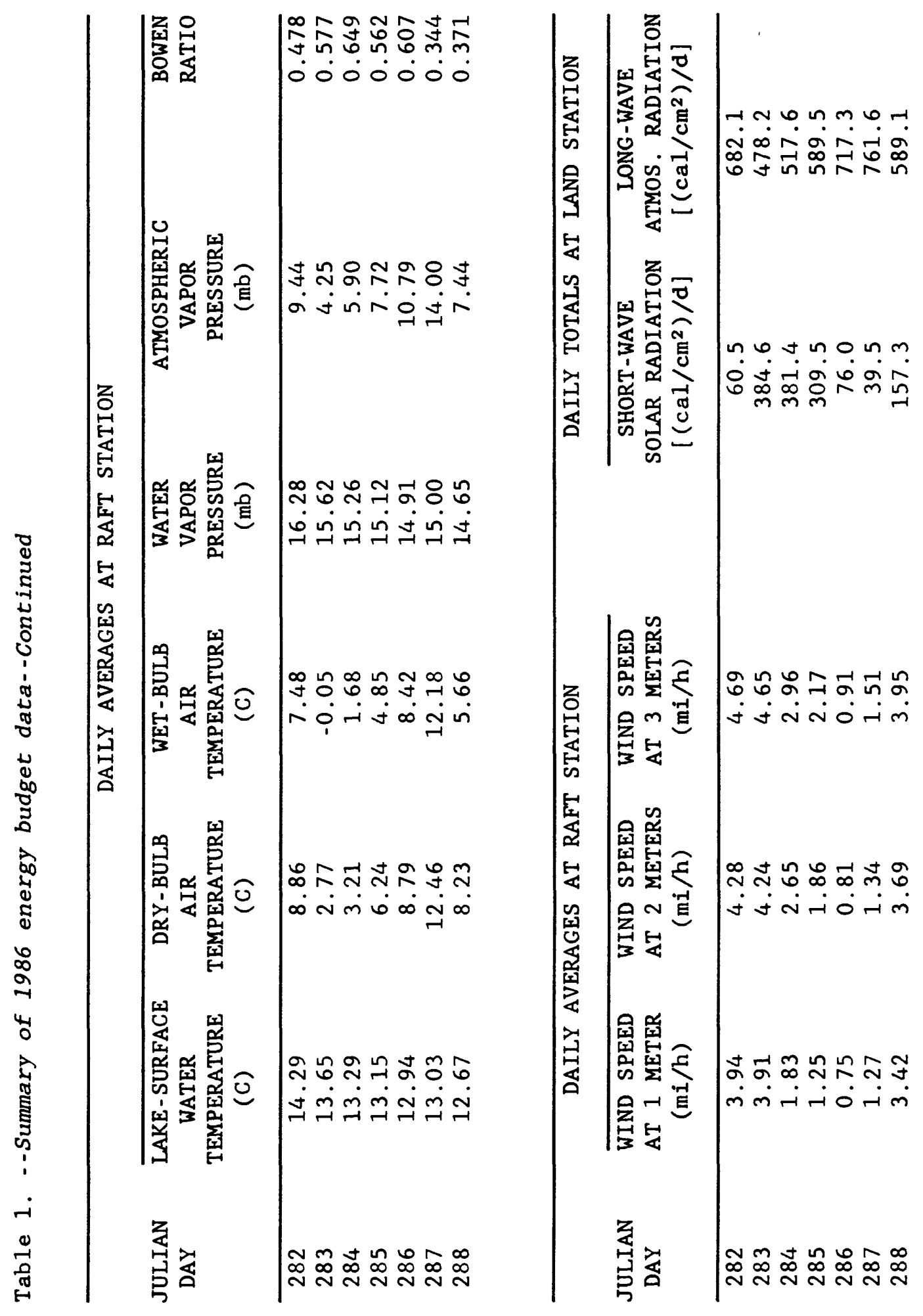




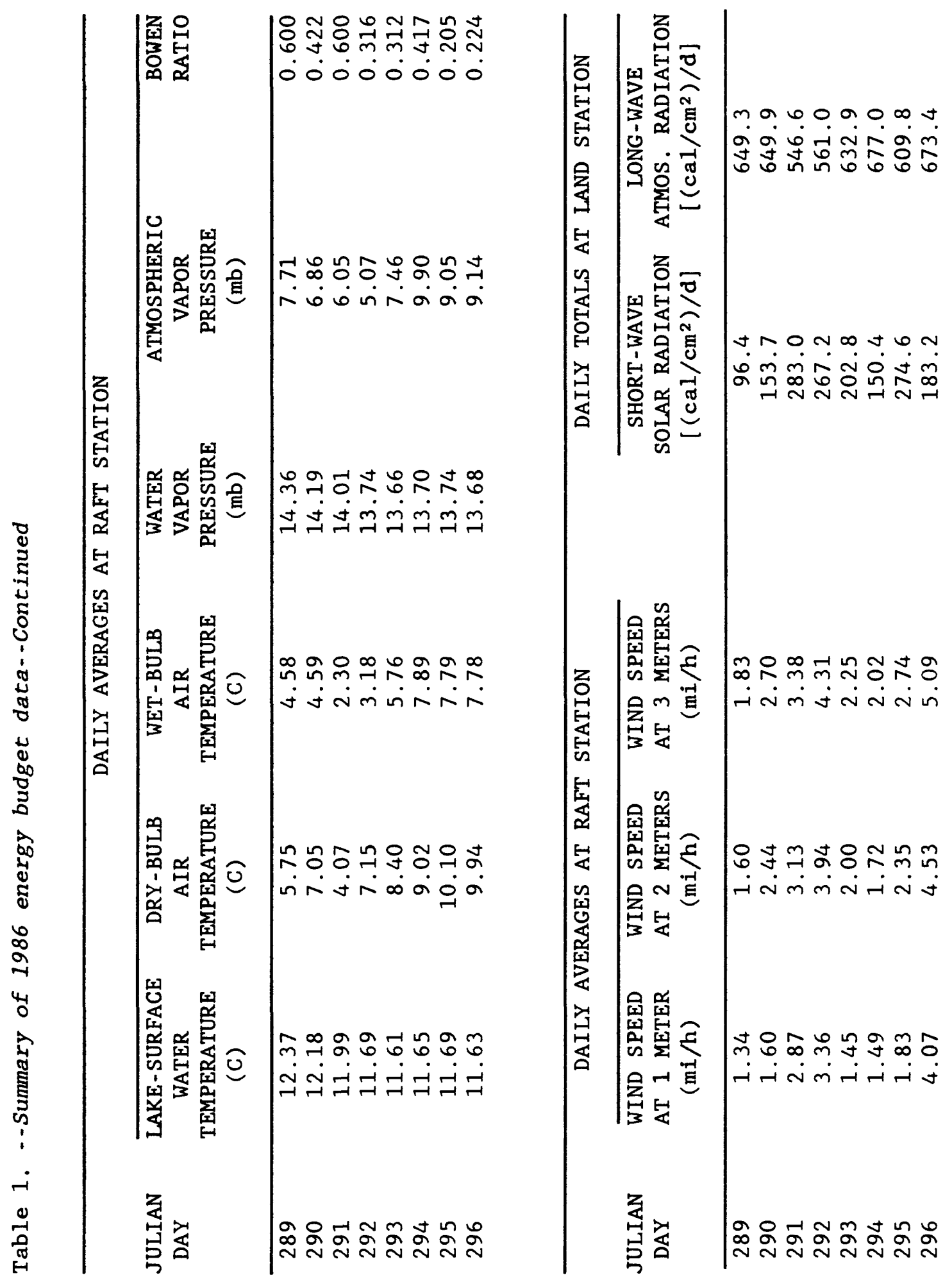




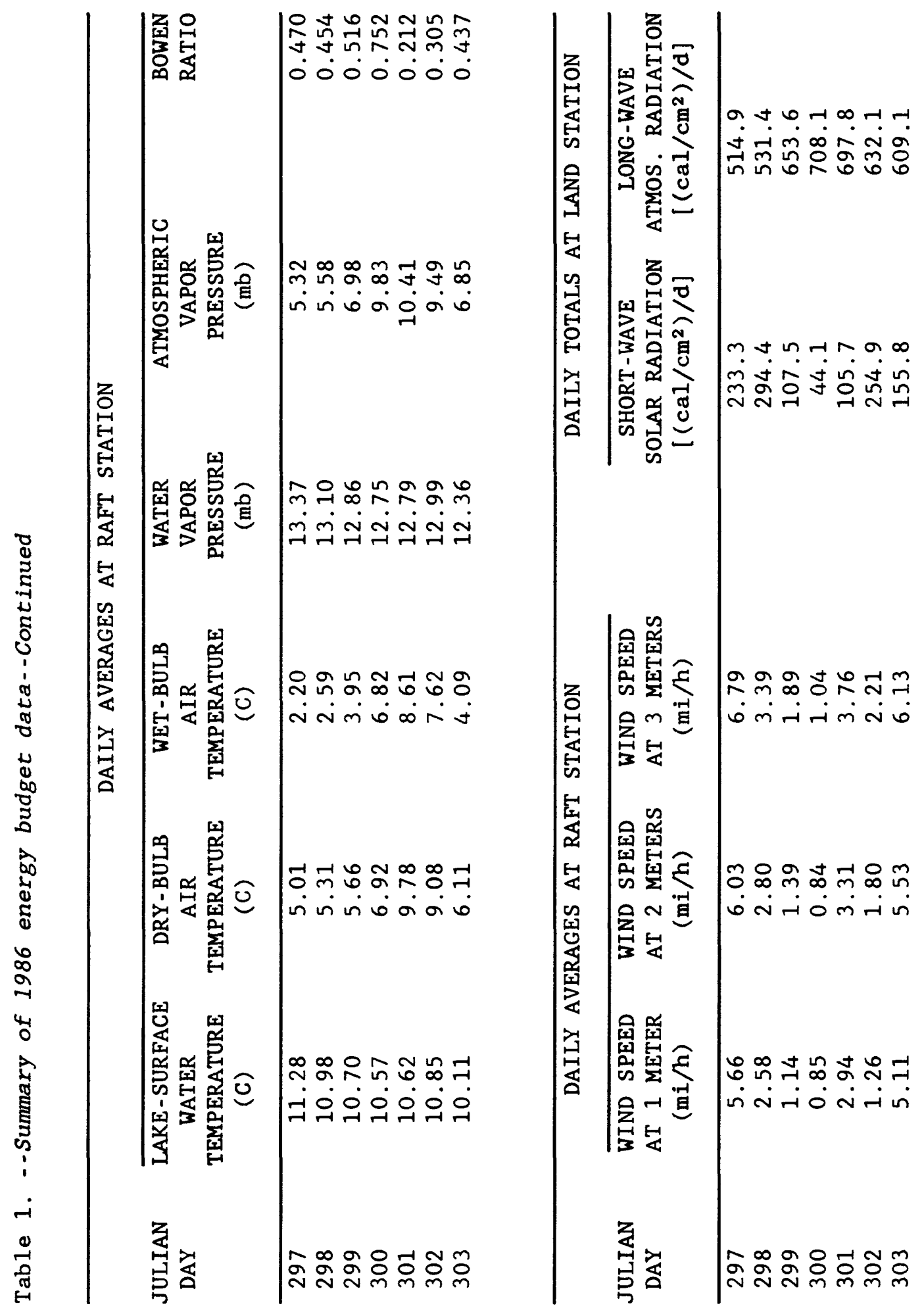




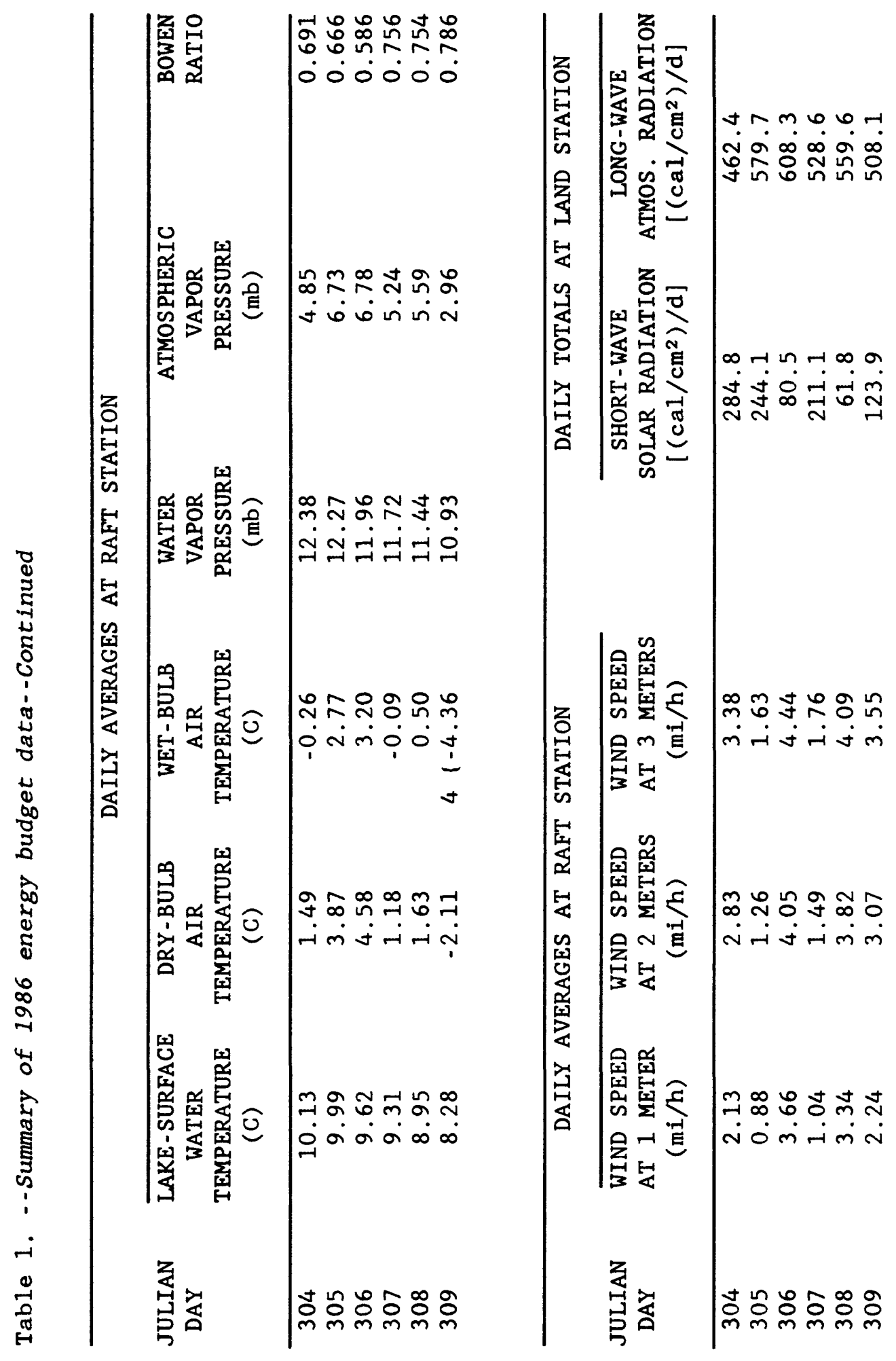




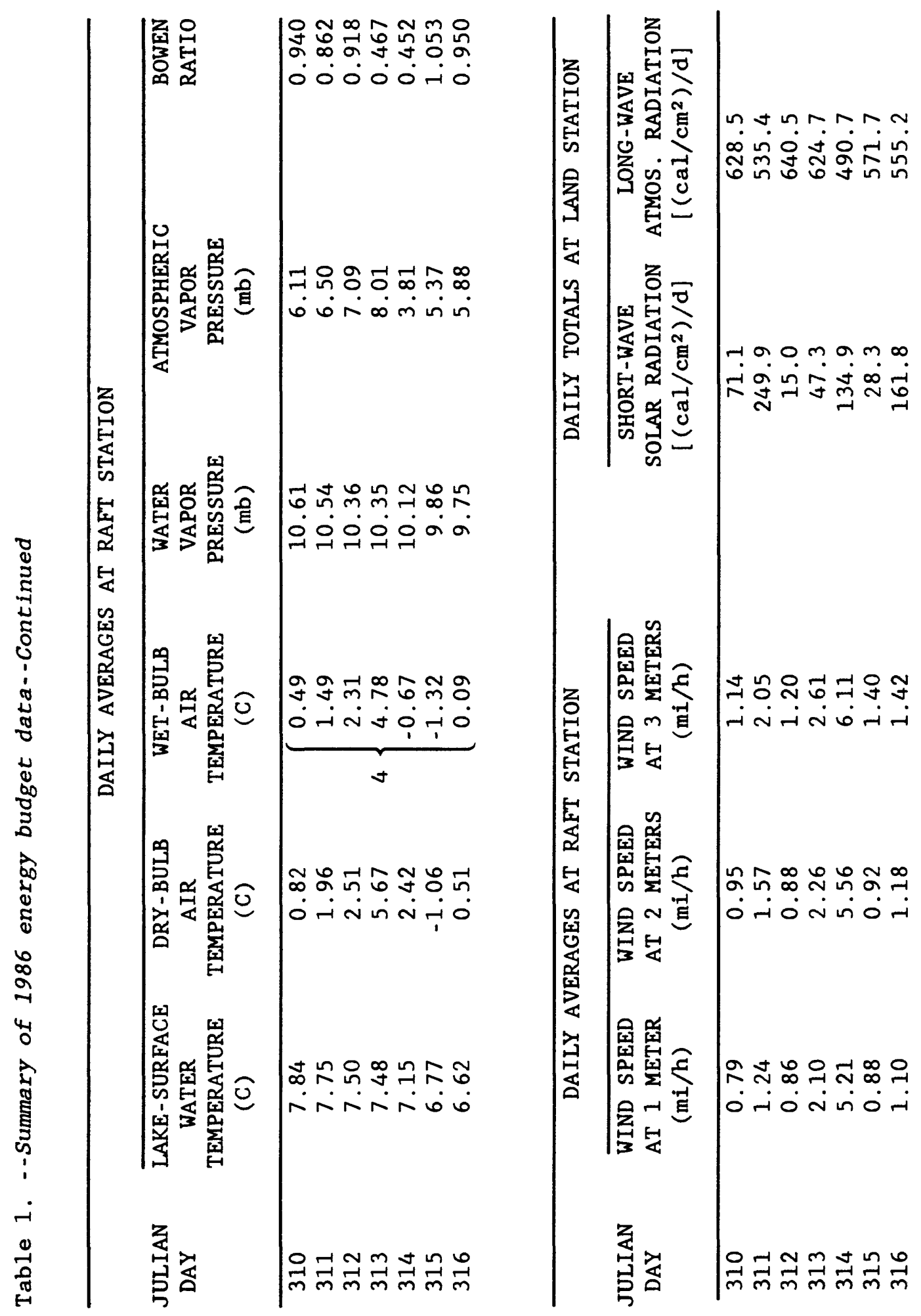




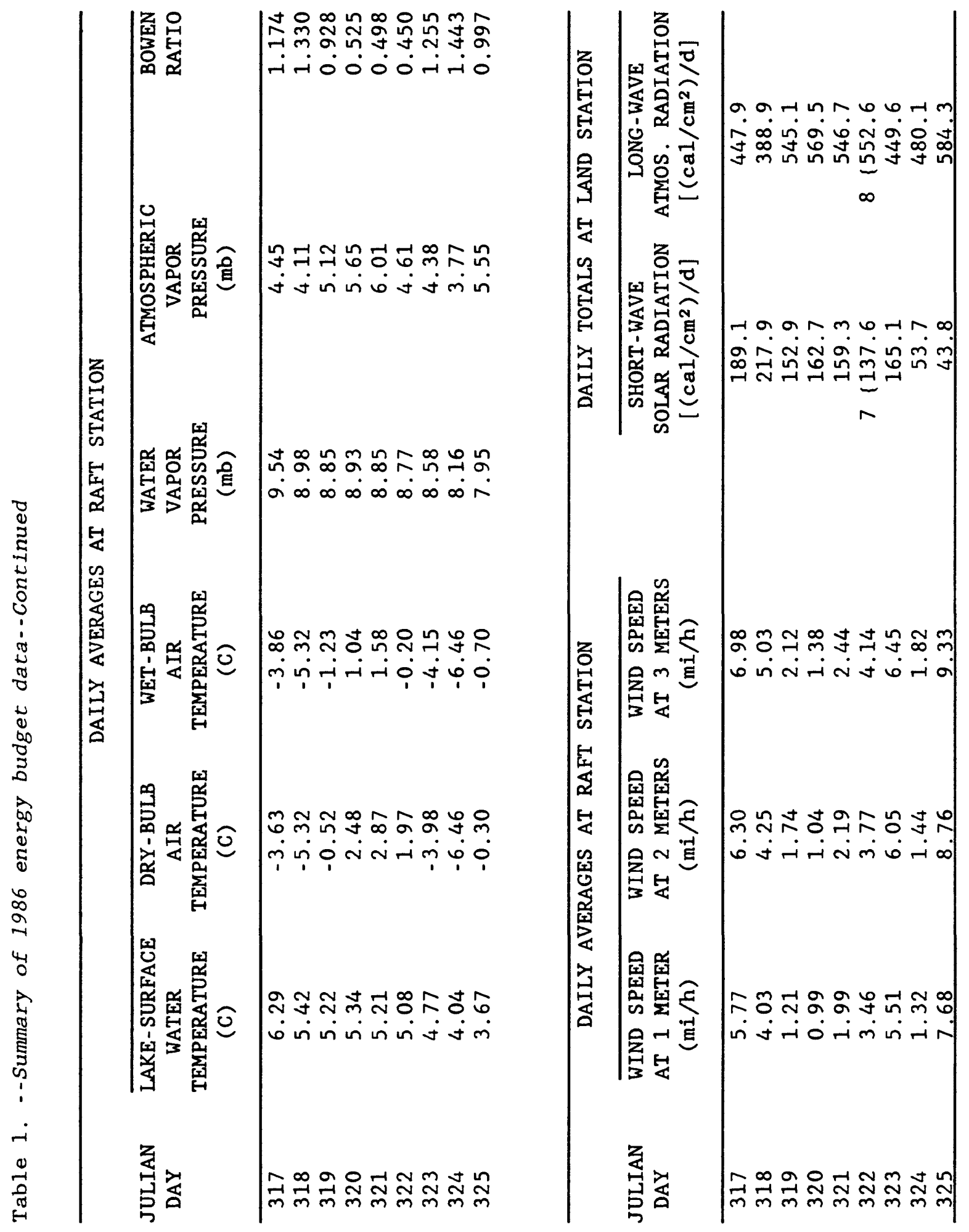


Footnotes to table 1:

1. Measured at 2 meters above water surface.

2. Water-vapor pressure is calculated using surface water-temperature data and assuming the air is completely saturated with water at the airwater interface.

3. Elevation above water surface.

4. Wet-bulb air temperature (Y) was calculated by regression equation 1 (see below), which was determined by using relative humidity data (X) from the hygrothermograph, the pyschrometric tables, and the wet-bulb temperature sensor connected to the digital data logger.

5. Water temperatures from 1600 hours on Julian day 168 to 1100 hours on Julian day 169 were estimated from a plot of temperatures typical of evening, night, and morning temperatures characteristic of that time of year and similar climatic conditions.

6. Dry-bulb temperature (Y) was calculated by regression equation 2 (see below), which was determined by using air-temperature data (X) from the hygrothermograph and the dry-bulb temperature sensor connected to the digital data logger.

7. Short-wave radiation ( $Y$ ) was calculated by regression equation 3 (see below), which was determined by using radiation data (X) collected by the U. S. Forest Service at the Hubbard Brook Field Headquarters and radiation data collected by the U. S. Geological Survey at the same location.

8. Long-wave radiation $=s \cdot T^{4}\left(c+d \sqrt{e_{a}}\right)$

where $s$ - Stephan $-4^{-B o l t z m a n}$ constant, $5.6697 \times 10^{-5}$ erg per square centimeter per second $\left({ }^{\circ} \mathrm{K}\right)$

$$
\begin{aligned}
T & =\text { air temperature, Kelvin } \\
c & =\text { constant (determined graphically) } \\
d & =0.0263 \\
e_{a} & =\text { vapor pressure of air. }
\end{aligned}
$$

Regression

Data points

$r^{2}$ value

1. $Y=-0.012+0.964 X$

2. $Y=0.608+1.010 X$

3. $Y=-5.00+24.00 X$
28

16

91
0.98

0.99

0.99 


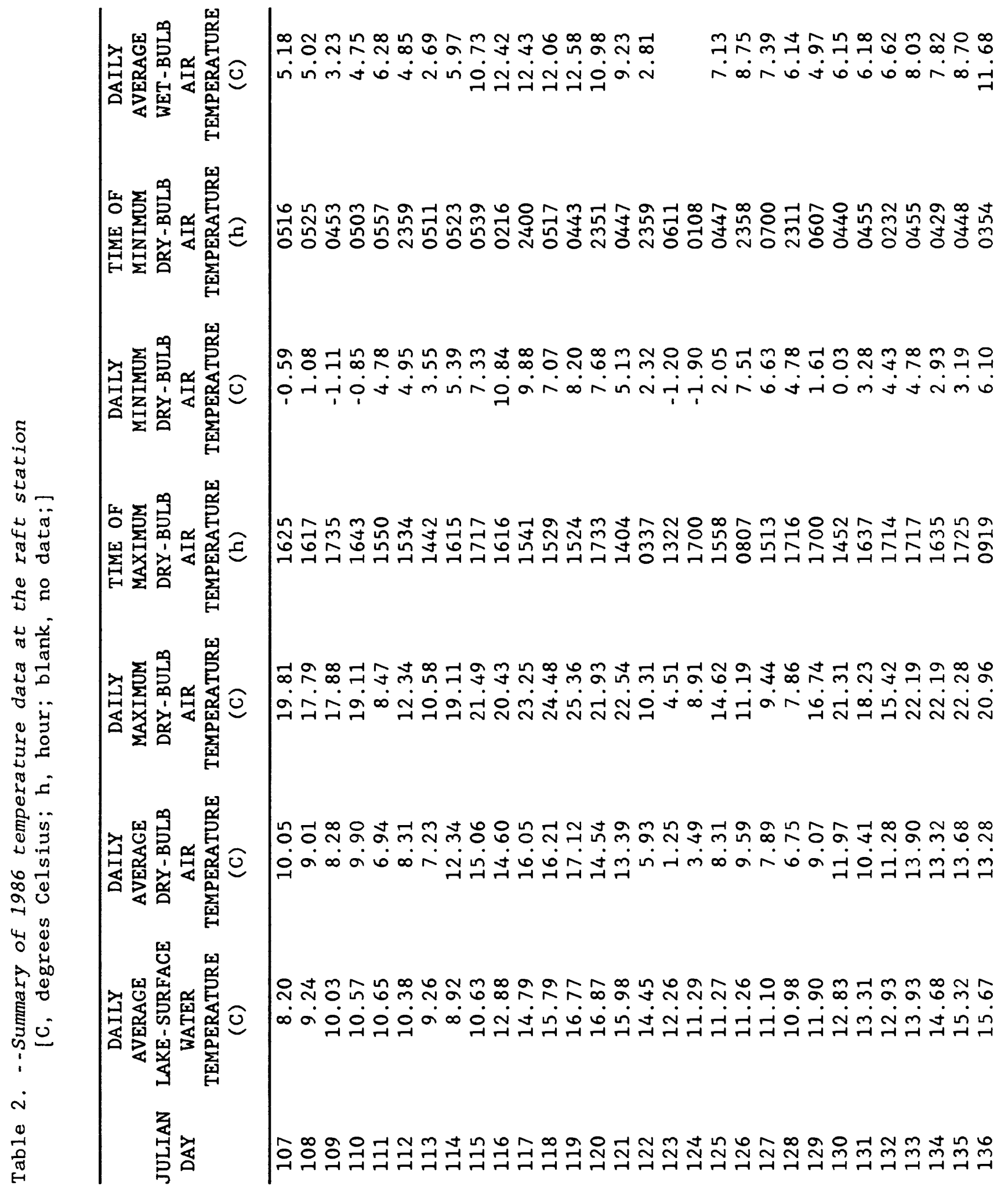


กำกำก

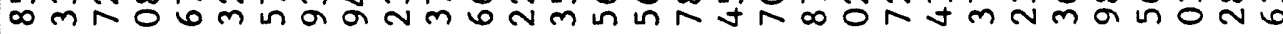

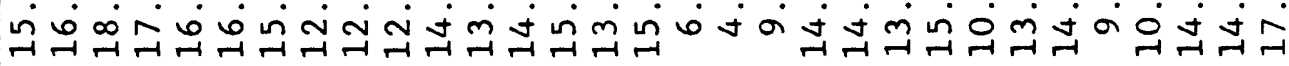

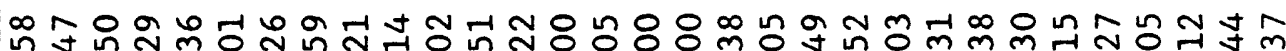

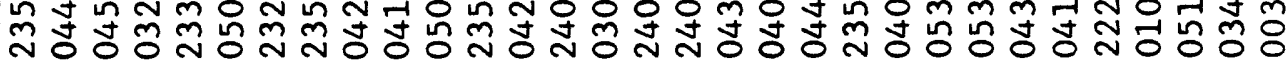

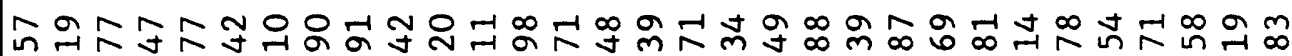

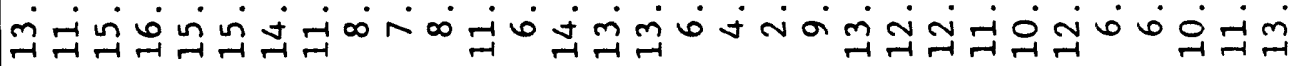

NOHG-mm กำ

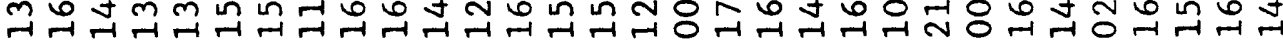

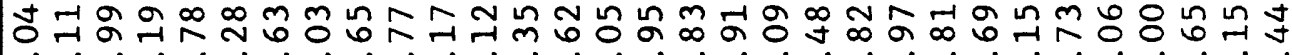

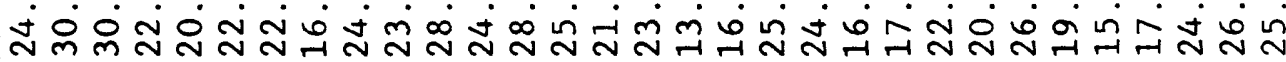

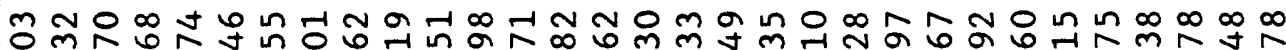

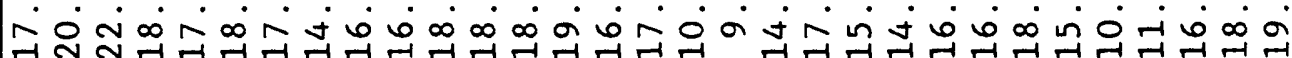

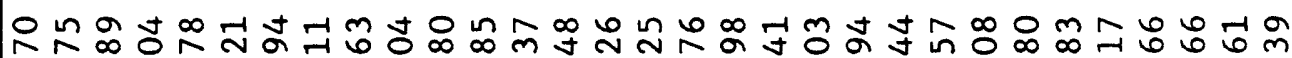

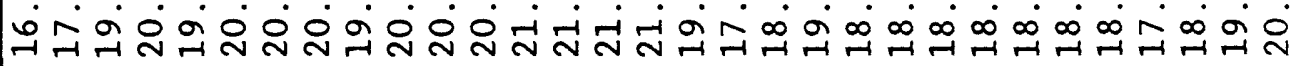

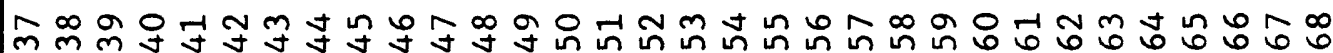

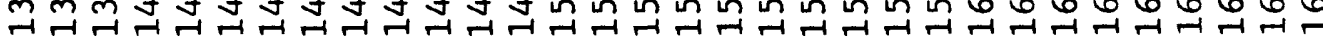


ษ

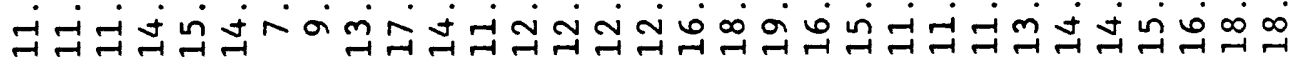

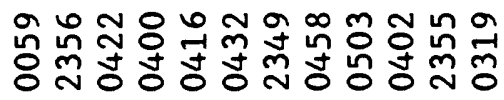

ำ 요 0

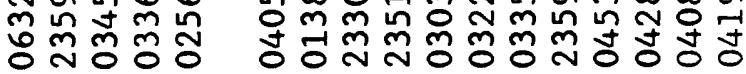

ํํำ유ำ

กิ

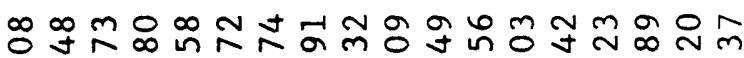

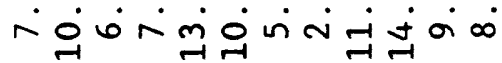

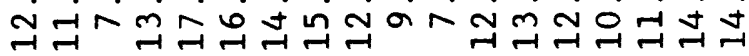

-

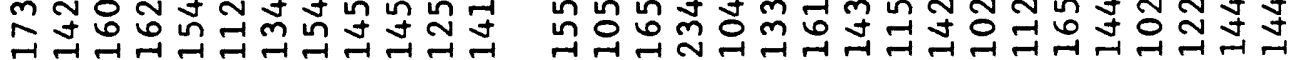

กํㅇำ

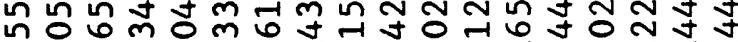

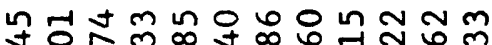

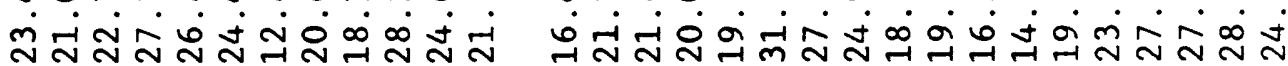
ษ

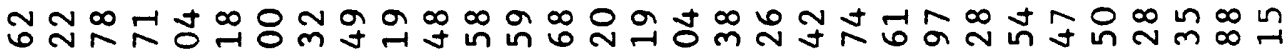

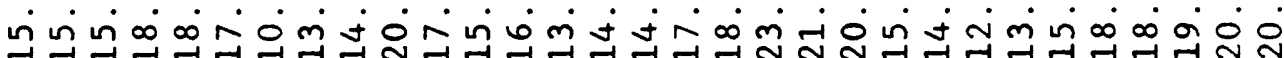

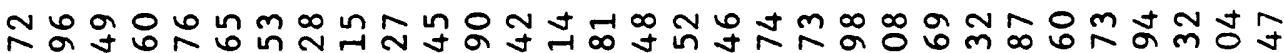

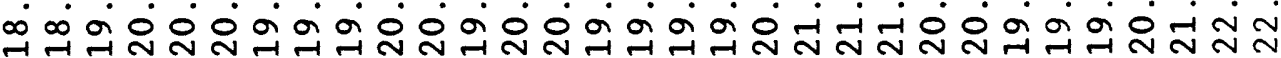

a O HNm th

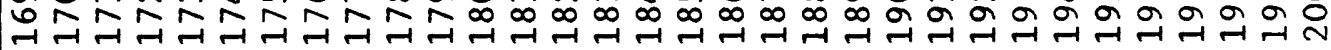




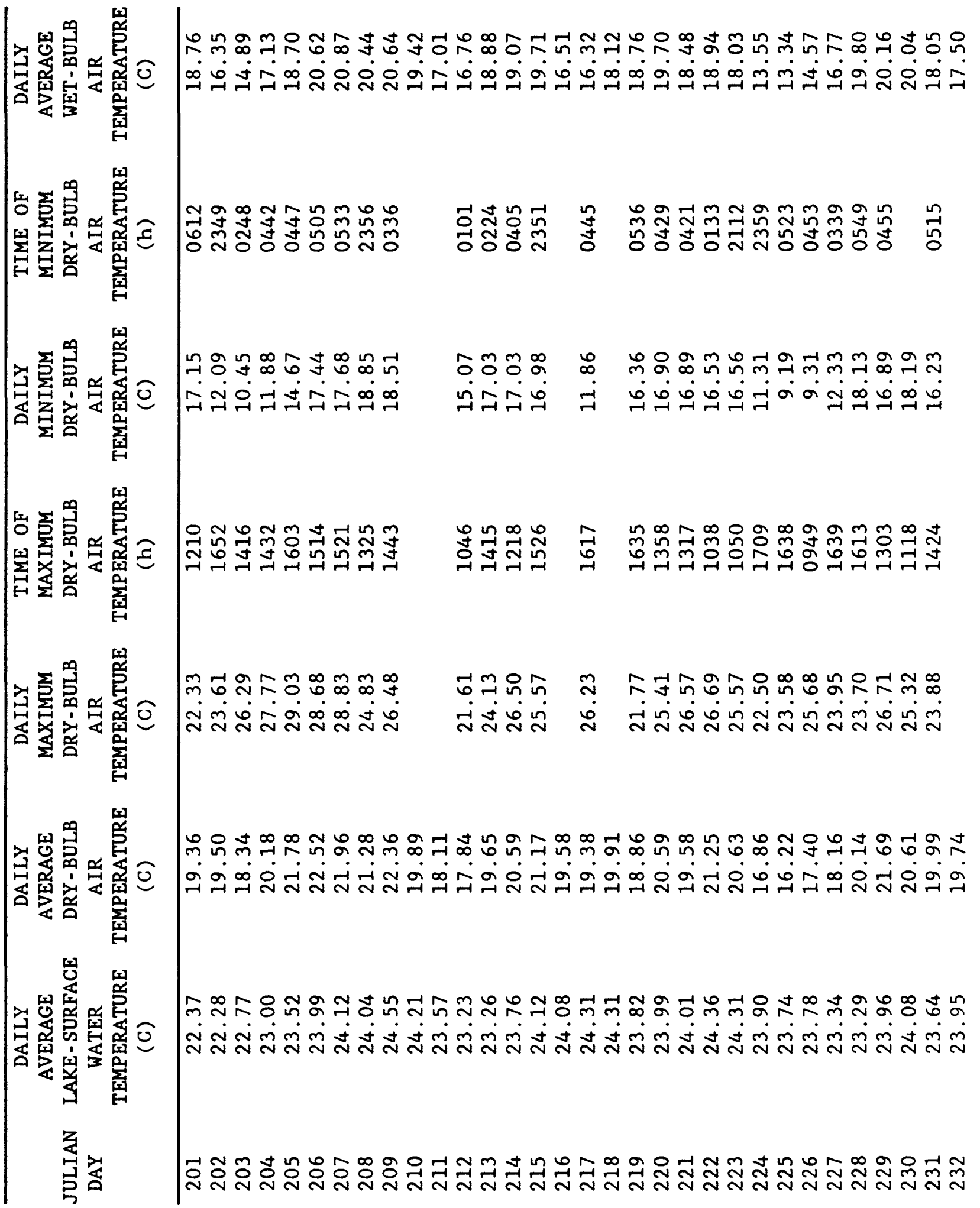




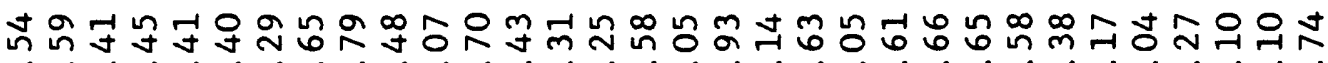

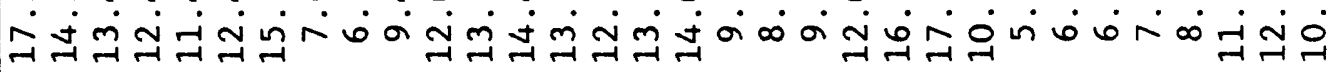

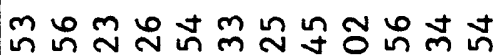

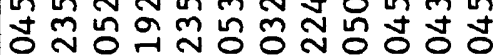

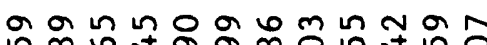
in $\infty$ o y o o $\infty$ in $y$ in

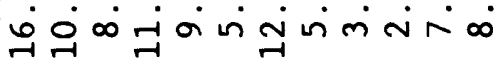

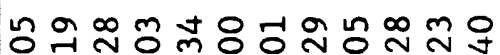
ํํㅇ I ก

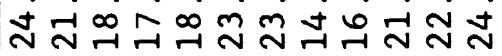

\section{ๆ

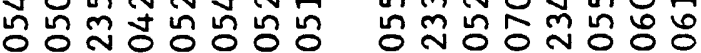

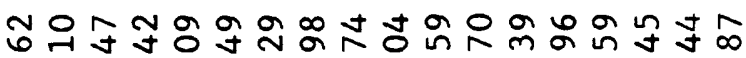

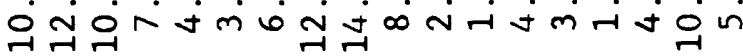

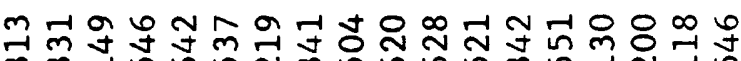

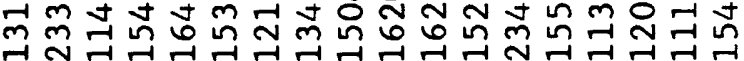

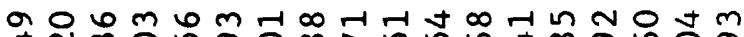

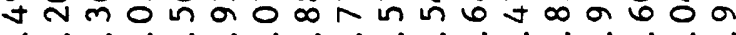
जि

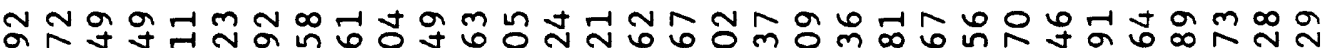

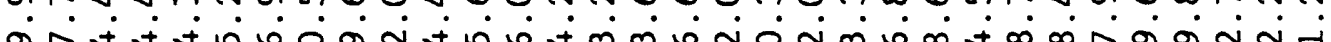

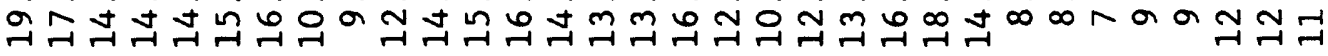

들

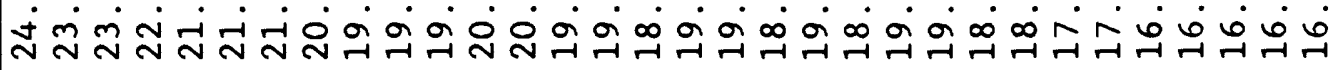

 
T $\infty$ Ho 0 o 0 N -

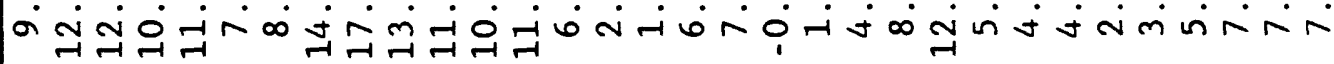

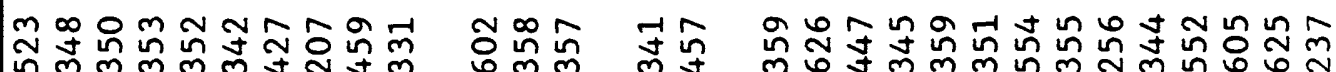

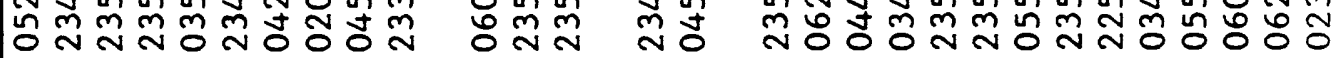

곡ำ

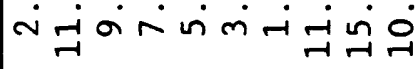

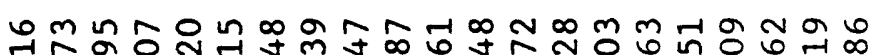
ம்iñ்

-1 -1 웅 ન્-

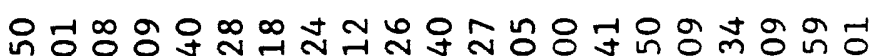

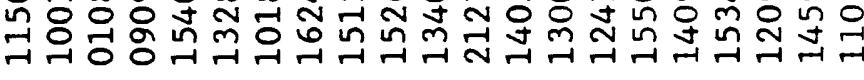

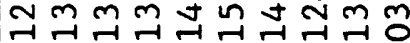

오융ํำำ t.m. 元

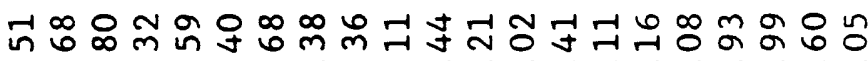

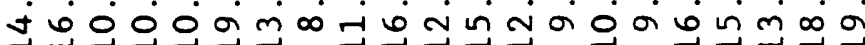

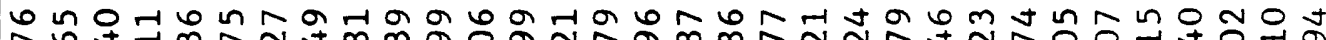

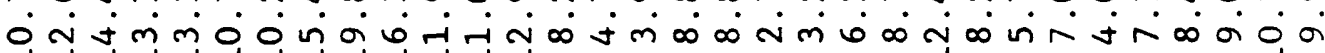

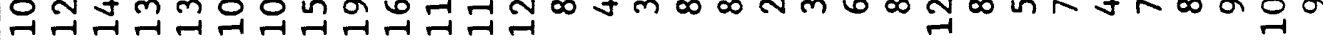

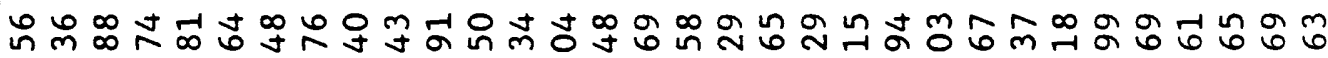

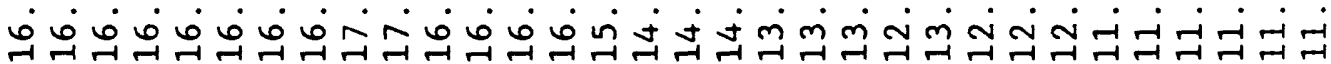

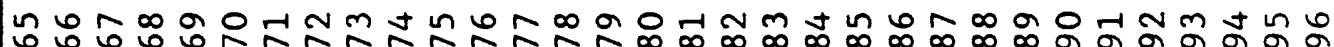

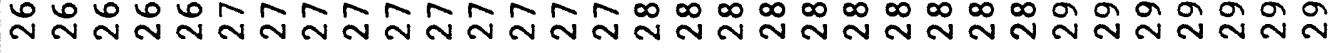




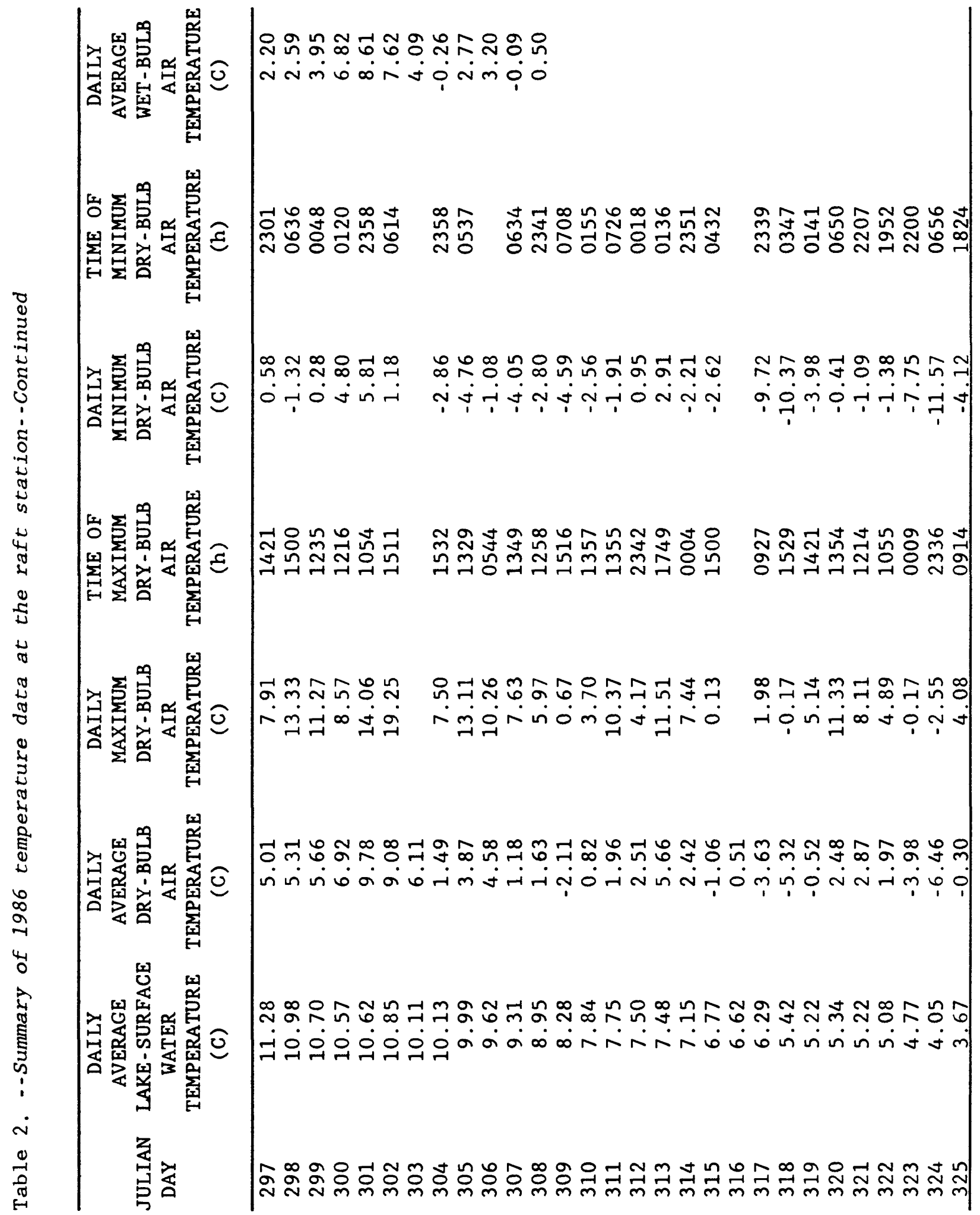


Table 3. --Summary of 1986 wind-speed data at the raft station

[m, meters; $\mathrm{mi} / \mathrm{h}$, miles per hour; $\mathrm{h}$, hour; blank, no data;]

\begin{tabular}{|c|c|c|c|c|c|c|}
\hline $\begin{array}{l}\text { HEIGHT OF } \\
\text { ANEMOMETER } \\
\text { ABOVE LAKE } \\
\text { SURFACE } \\
\text { (m) }\end{array}$ & $\begin{array}{l}\text { JULIAN } \\
\text { DAY }\end{array}$ & $\begin{array}{l}\text { DAILY } \\
\text { AVERAGE } \\
\text { WIND } \\
\text { SPEED } \\
(\mathrm{mi} / \mathrm{h})\end{array}$ & $\begin{array}{l}\text { DAILY } \\
\text { MAXIMUM } \\
\text { WIND } \\
\text { SPEED } \\
(\mathrm{mi} / \mathrm{h})\end{array}$ & $\begin{array}{l}\text { TIME OF } \\
\text { MAXIMUM } \\
\text { WIND } \\
\text { SPEED } \\
\text { (h) }\end{array}$ & $\begin{array}{l}\text { DAILY } \\
\text { MINIMUM } \\
\text { WIND } \\
\text { SPEED } \\
(\mathrm{mi} / \mathrm{h})\end{array}$ & $\begin{array}{c}\text { TIME OF } \\
\text { MINIMUM } \\
\text { WIND } \\
\text { SPEED } \\
\text { (h) }\end{array}$ \\
\hline 1 & 107 & 2.29 & 17.35 & 1344 & 0.60 & 1957 \\
\hline 1 & 108 & 2.54 & 17.86 & 1406 & 0.60 & 2017 \\
\hline 1 & 109 & 2.45 & 12.85 & 1421 & 0.60 & 0730 \\
\hline 1 & 110 & 2.75 & 15.54 & 1354 & 0.60 & 2359 \\
\hline 1 & 111 & 1.76 & 12.96 & 1528 & 0.60 & 0719 \\
\hline 1 & 112 & 6.95 & 26.89 & 2312 & 0.60 & 1845 \\
\hline 1 & 113 & 8.34 & 30.62 & 1602 & 0.60 & 2232 \\
\hline 1 & 114 & 7.04 & 28.67 & 0817 & 0.60 & 2232 \\
\hline 1 & 115 & 3.23 & 16.81 & 1507 & 0.60 & 2359 \\
\hline 1 & 116 & 1.56 & 9.68 & 1401 & 0.60 & 1821 \\
\hline 1 & 117 & 2.16 & 13.42 & 1552 & 0.60 & 1728 \\
\hline 1 & 118 & 2.43 & 12.06 & 1504 & 0.60 & 2250 \\
\hline 1 & 119 & 2.63 & 13.05 & 1535 & 0.60 & 0749 \\
\hline 1 & 120 & 2.17 & 24.25 & 1506 & 0.60 & 0645 \\
\hline 1 & 121 & 3.81 & 27.40 & 2014 & 0.60 & 2302 \\
\hline 1 & 122 & 6.18 & 25.78 & 1341 & 0.60 & 0120 \\
\hline 1 & 123 & 7.52 & 27.08 & 0427 & 0.60 & 2400 \\
\hline 1 & 124 & 5.41 & 31.87 & 0628 & 0.60 & 2150 \\
\hline 1 & 125 & 1.85 & 11.92 & 2102 & 0.60 & 1603 \\
\hline 1 & 126 & 1.71 & 10.81 & 1131 & 0.60 & 2356 \\
\hline 1 & 127 & 1.37 & 5.75 & 0918 & 0.60 & 1430 \\
\hline 1 & 128 & 1.20 & 7.73 & 1153 & 0.60 & 2122 \\
\hline 1 & 129 & 2.27 & 15.23 & 1447 & 0.60 & 2352 \\
\hline 1 & 130 & 2.78 & 15.91 & 1404 & 0.60 & 0626 \\
\hline 1 & 131 & 2.41 & 12.45 & 1421 & 0.60 & 2310 \\
\hline 1 & 132 & 3.19 & 17.46 & 1050 & 0.60 & 0452 \\
\hline 1 & 133 & 2.34 & 12.08 & 1456 & 0.60 & 2213 \\
\hline 1 & 134 & 2.90 & 14.09 & 1709 & 0.60 & 2218 \\
\hline 1 & 135 & 2.90 & 16.30 & 1324 & 0.60 & 2142 \\
\hline 1 & 136 & 1.14 & 9.85 & 1128 & 0.60 & 1027 \\
\hline 1 & 137 & 0.97 & 9.23 & 1223 & 0.60 & 2323 \\
\hline 1 & 138 & 2.73 & 14.38 & 1206 & 0.60 & 0617 \\
\hline 1 & 139 & 2.15 & 17.07 & 1425 & 0.60 & 1828 \\
\hline 1 & 140 & 1.93 & 12.00 & 1247 & 0.60 & 1907 \\
\hline 1 & 141 & 1.93 & 13.44 & 1159 & 0.60 & 1703 \\
\hline 1 & 142 & 2.67 & 13.25 & 1522 & 0.60 & 0332 \\
\hline 1 & 143 & 2.64 & 14.69 & 1207 & 0.60 & 1902 \\
\hline 1 & 144 & 3.01 & 19.33 & 1147 & 0.60 & 2357 \\
\hline 1 & 145 & 3.08 & 16.90 & 0757 & 0.60 & 2057 \\
\hline 1 & 146 & 2.57 & 13.73 & 1423 & 0.60 & 0551 \\
\hline 1 & 147 & 2.42 & 16.87 & 1207 & 0.60 & 0725 \\
\hline 1 & 148 & 4.95 & 25.30 & 1116 & 0.60 & 2400 \\
\hline 1 & 149 & 2.10 & 16.70 & 1132 & 0.60 & 0857 \\
\hline 1 & 150 & 3.38 & 16.73 & 0733 & 0.60 & 2208 \\
\hline 1 & 151 & 3.76 & 19.24 & 1322 & 0.60 & 2326 \\
\hline 1 & 152 & 3.08 & 19.61 & 2324 & 0.60 & 2321 \\
\hline 1 & 153 & 6.99 & 24.28 & 0122 & 0.60 & 2159 \\
\hline
\end{tabular}


Table 3. --Summary of 1986 wind-speed data at the raft station--Continued

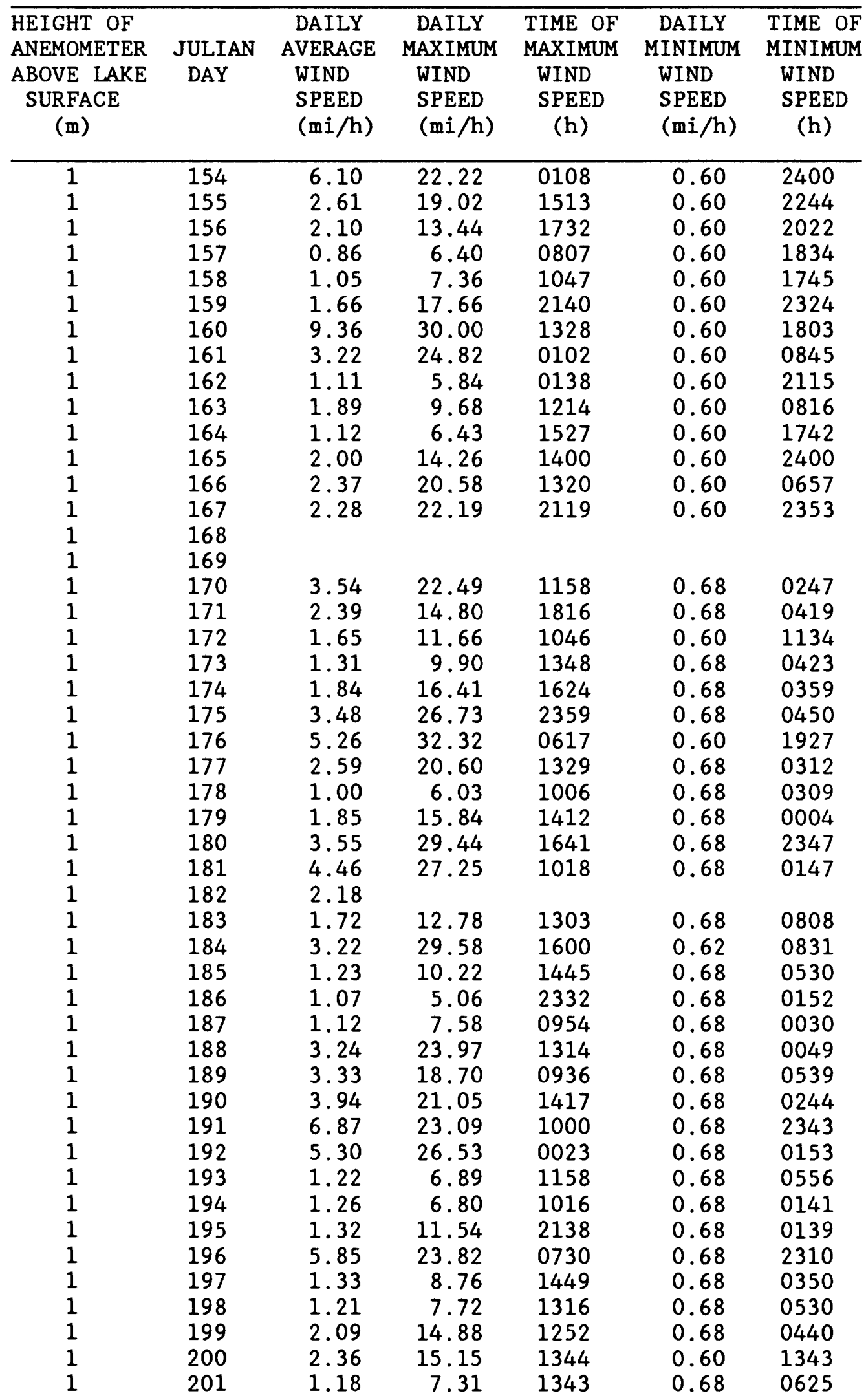


Table 3. --Summary of 1986 wind-speed data at the raft station--Continued

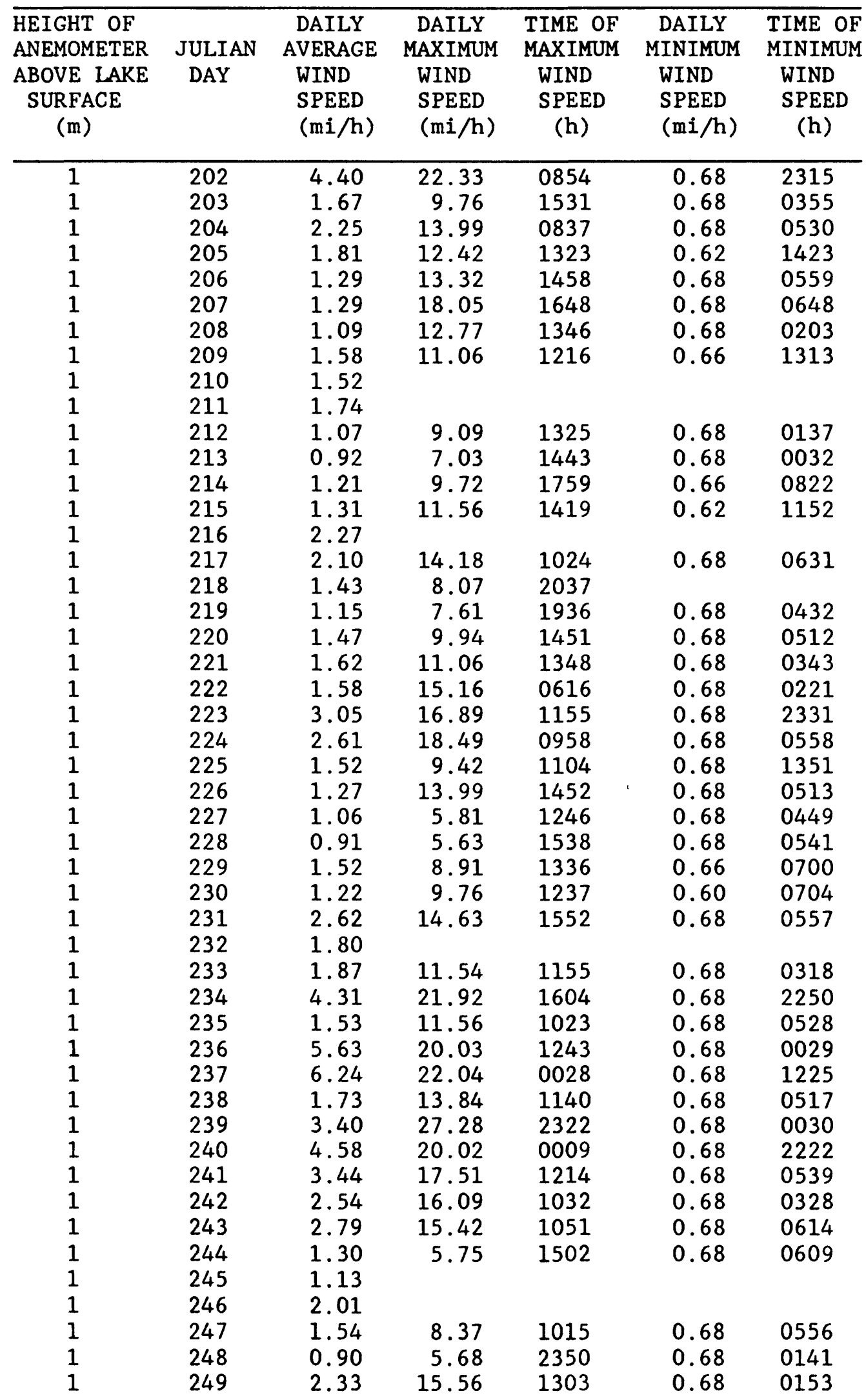


Table 3. --Summary of 1986 wind-speed data at the raft station--Continued

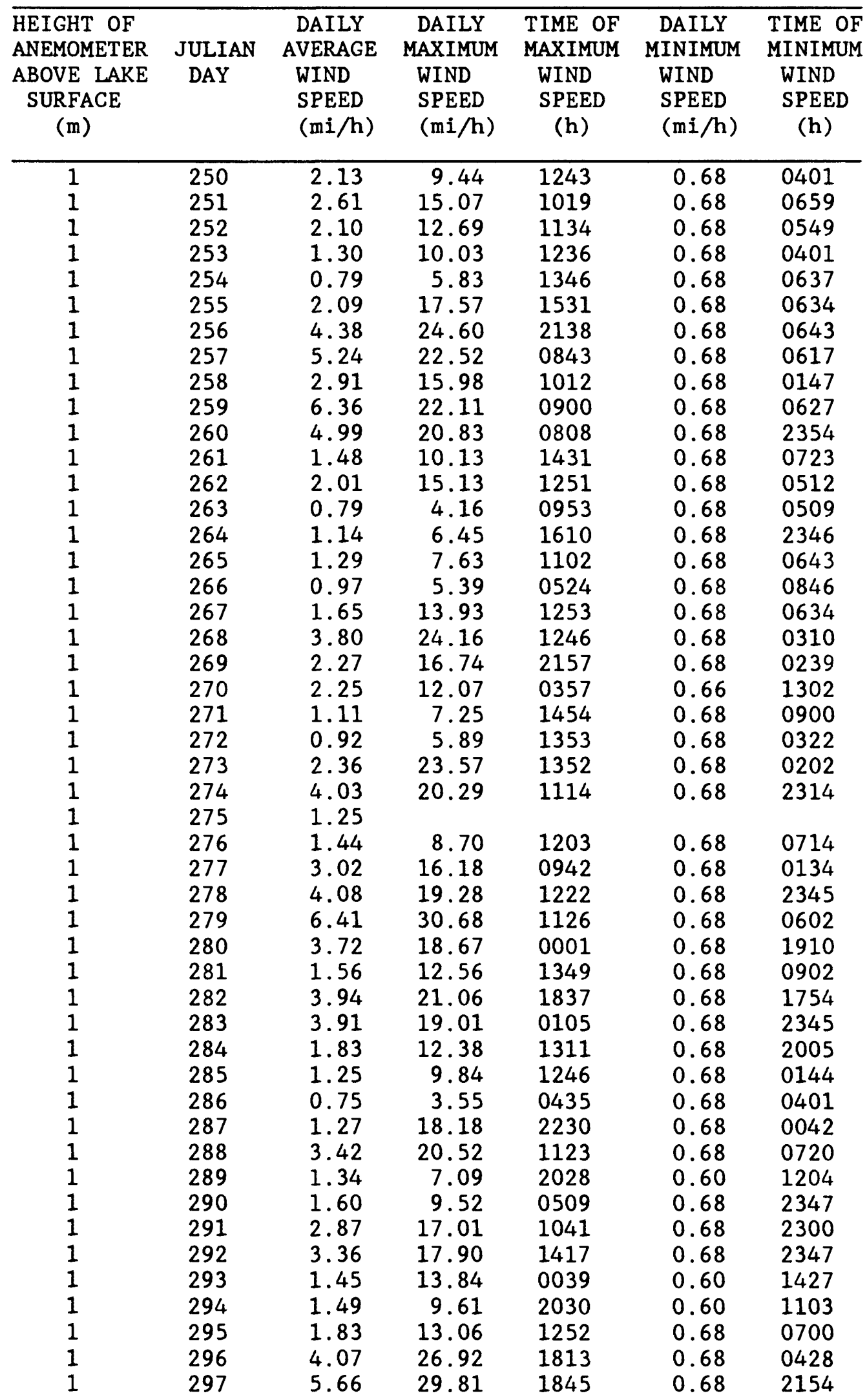


Table 3. --Summary of 1986 wind-speed data at the raft station--Continued

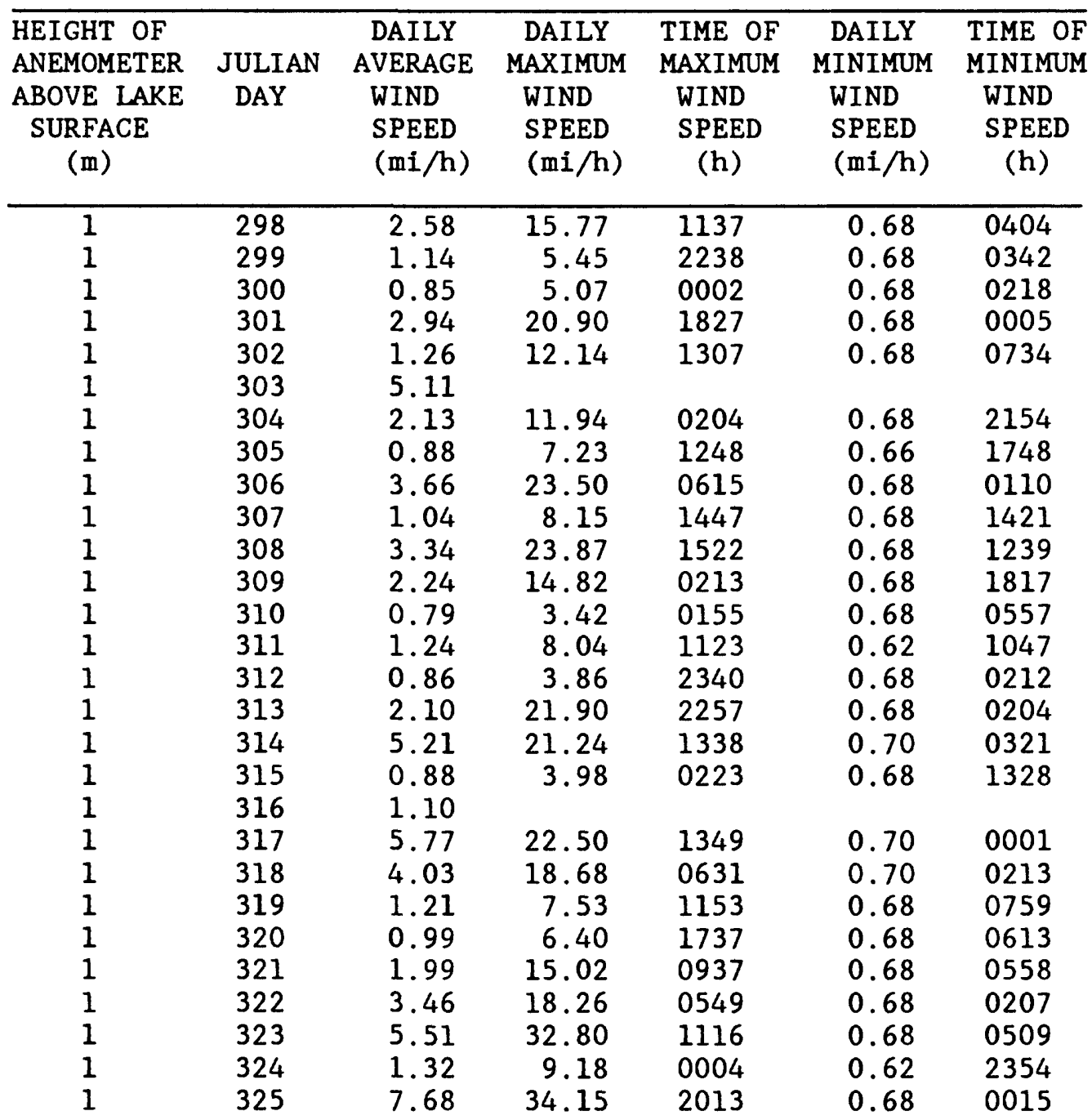


Table 3. --Summary of 1986 wind-speed data at the raft station--Continued

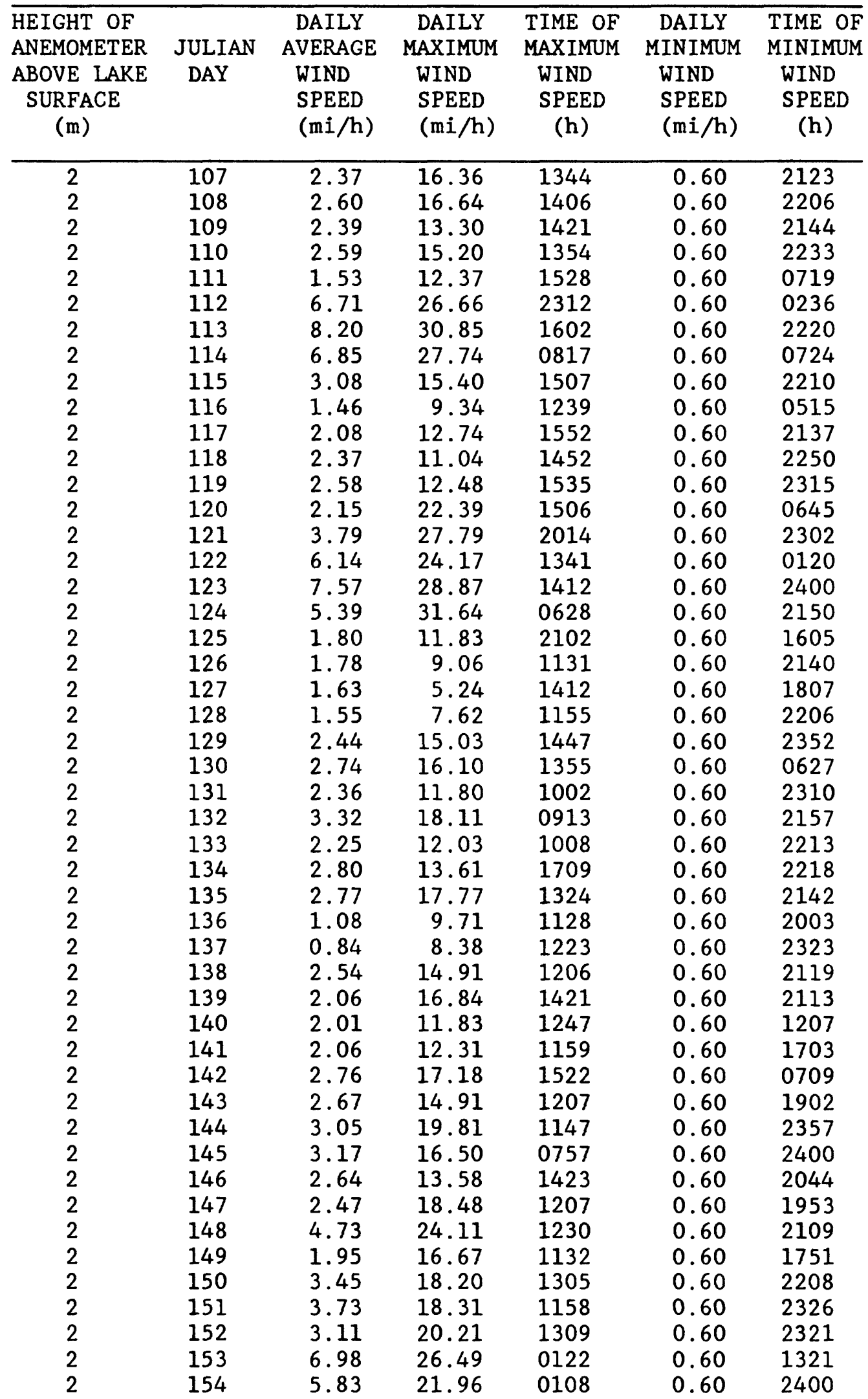


Table 3. --Summary of 1986 wind-speed data at the raft station--Continued

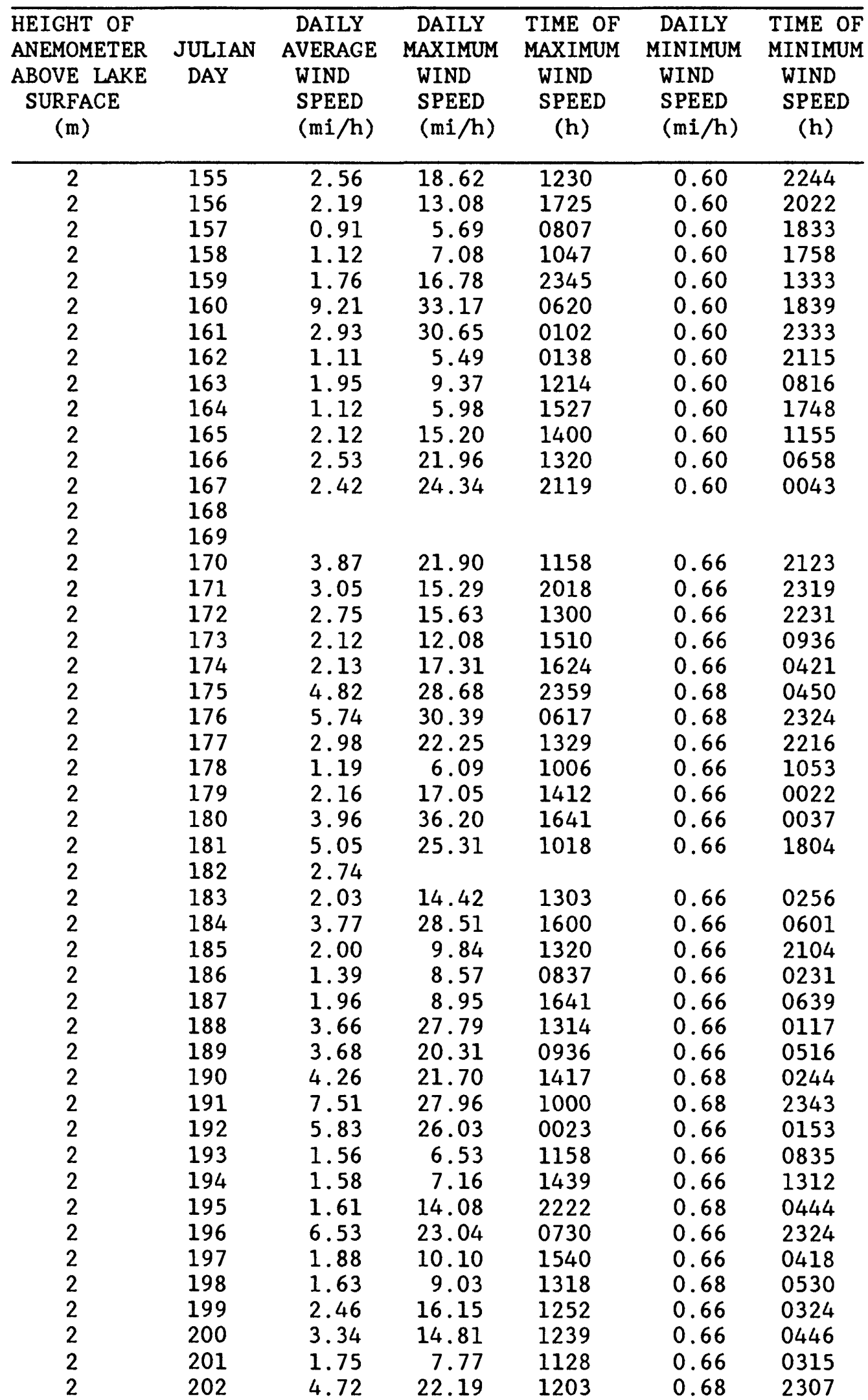


Table 3. --Summary of 1986 wind-speed data at the raft station--Continued

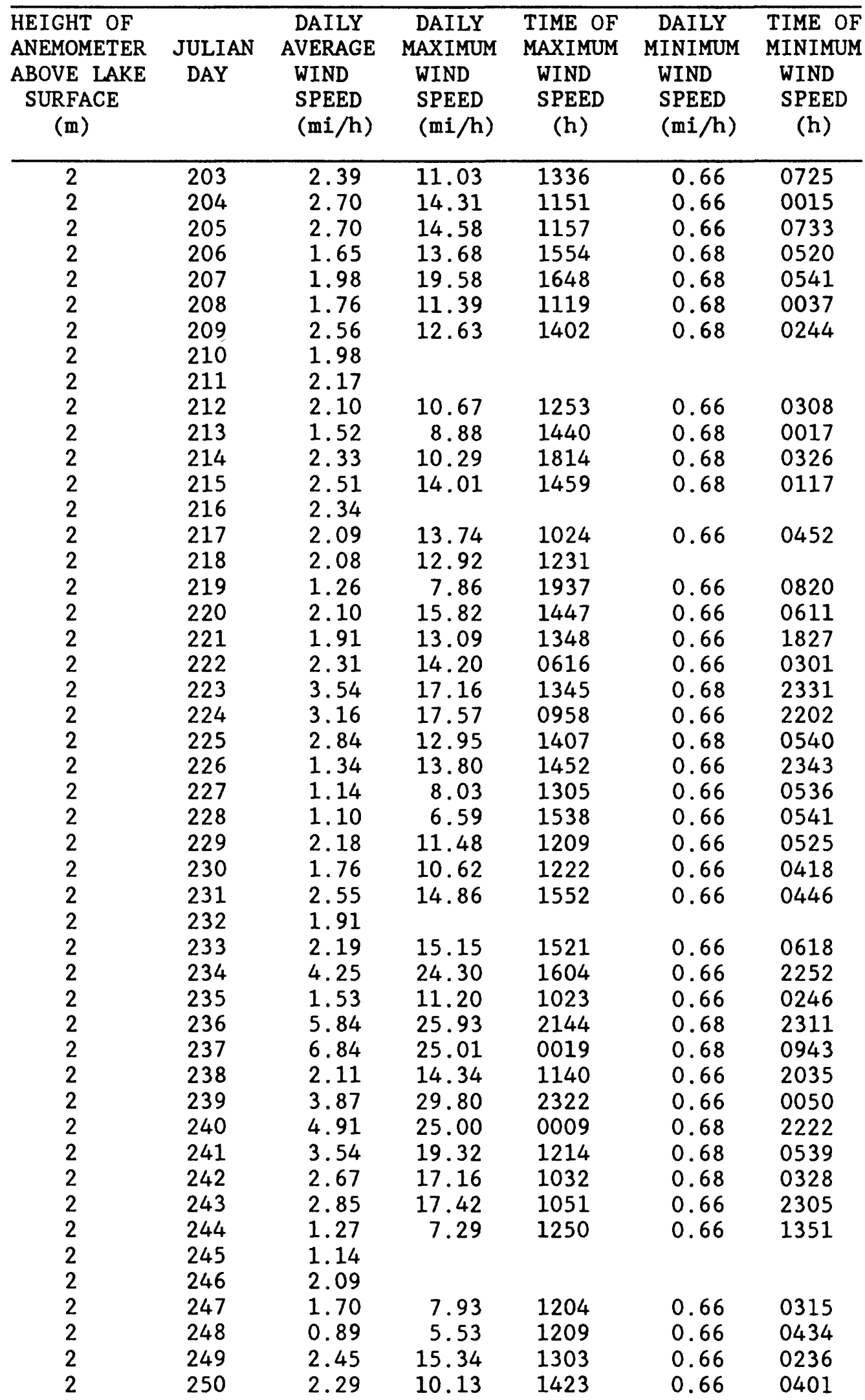


Table 3. --Summary of 1986 wind-speed data at the raft station--Continued

\begin{tabular}{|c|c|c|c|c|c|c|}
\hline $\begin{array}{l}\text { HEIGHT OF } \\
\text { ANEMOMETER } \\
\text { ABOVE LAKE } \\
\text { SURFACE } \\
\text { (m) }\end{array}$ & $\begin{array}{l}\text { JULIAN } \\
\text { DAY }\end{array}$ & $\begin{array}{l}\text { DAILY } \\
\text { AVERAGE } \\
\text { WIND } \\
\text { SPEED } \\
(\mathrm{mi} / \mathrm{h})\end{array}$ & $\begin{array}{l}\text { DAILY } \\
\text { MAXIMUM } \\
\text { WIND } \\
\text { SPEED } \\
(\mathrm{mi} / \mathrm{h})\end{array}$ & $\begin{array}{l}\text { TIME OF } \\
\text { MAXIMUM } \\
\text { WIND } \\
\text { SPEED } \\
\text { (h) }\end{array}$ & $\begin{array}{l}\text { DAILY } \\
\text { MINIMUM } \\
\text { WIND } \\
\text { SPEED } \\
(\mathrm{mi} / \mathrm{h})\end{array}$ & $\begin{array}{l}\text { TIME OF } \\
\text { MINIMUM } \\
\text { WIND } \\
\text { SPEED } \\
\text { (h) }\end{array}$ \\
\hline $\begin{array}{l}2 \\
2 \\
2 \\
2 \\
2 \\
2 \\
2 \\
2 \\
2 \\
2 \\
2 \\
2 \\
2 \\
2 \\
2 \\
2 \\
2 \\
2 \\
2 \\
2 \\
2 \\
2 \\
2 \\
2\end{array}$ & $\begin{array}{l}251 \\
252 \\
253 \\
254 \\
255 \\
256 \\
257 \\
258 \\
259 \\
260 \\
261 \\
262 \\
263 \\
264 \\
265 \\
266 \\
267 \\
268 \\
269 \\
270 \\
271 \\
272 \\
273 \\
274\end{array}$ & $\begin{array}{l}2.46 \\
2.01 \\
1.39 \\
0.79 \\
2.46 \\
4.63 \\
5.55 \\
2.65 \\
6.89 \\
5.28 \\
1.83 \\
2.15 \\
0.92 \\
1.45 \\
1.69 \\
1.14 \\
2.00 \\
2.32 \\
2.71 \\
2.61 \\
1.22 \\
1.09 \\
2.48 \\
4.43\end{array}$ & $\begin{array}{r}15.55 \\
13.42 \\
11.27 \\
5.16 \\
19.36 \\
25.66 \\
26.79 \\
17.89 \\
24.30 \\
23.70 \\
11.65 \\
17.22 \\
4.97 \\
6.23 \\
11.47 \\
6.33 \\
15.68 \\
26.64 \\
18.14 \\
13.55 \\
8.45 \\
7.92 \\
25.64 \\
21.49\end{array}$ & $\begin{array}{l}1019 \\
1134 \\
1154 \\
1346 \\
1531 \\
2138 \\
1023 \\
1339 \\
0900 \\
0808 \\
1207 \\
1304 \\
0613 \\
1610 \\
1035 \\
1826 \\
1253 \\
0918 \\
2124 \\
0357 \\
1331 \\
1258 \\
1352 \\
1003\end{array}$ & $\begin{array}{l}0.68 \\
0.68 \\
0.68 \\
0.68 \\
0.68 \\
0.68 \\
0.68 \\
0.68 \\
0.68 \\
0.68 \\
0.68 \\
0.68 \\
0.68 \\
0.68 \\
0.68 \\
0.68 \\
0.68 \\
0.68 \\
0.68 \\
0.68 \\
0.68 \\
0.68 \\
0.68 \\
0.68\end{array}$ & $\begin{array}{l}0619 \\
0549 \\
0401 \\
0559 \\
0634 \\
0605 \\
2212 \\
0113 \\
0627 \\
2346 \\
0309 \\
0512 \\
0459 \\
2346 \\
0643 \\
0833 \\
0634 \\
0310 \\
0146 \\
0643 \\
0900 \\
0255 \\
0202 \\
2246\end{array}$ \\
\hline 2 & 275 & 1.69 & & & & \\
\hline & $\begin{array}{l}276 \\
277\end{array}$ & $\begin{array}{l}1.86 \\
3.25\end{array}$ & $\begin{array}{l}17.22 \\
18.58\end{array}$ & $\begin{array}{l}1204 \\
0942\end{array}$ & $\begin{array}{l}0.68 \\
0.68\end{array}$ & $\begin{array}{l}0627 \\
0015\end{array}$ \\
\hline $\begin{array}{l}2 \\
2\end{array}$ & $\begin{array}{l}278 \\
279\end{array}$ & $\begin{array}{l}4.51 \\
7.28\end{array}$ & $\begin{array}{l}23.31 \\
34.34\end{array}$ & $\begin{array}{l}1123 \\
2105\end{array}$ & $\begin{array}{l}0.68 \\
0.62\end{array}$ & $\begin{array}{l}2358 \\
1534\end{array}$ \\
\hline 2 & 280 & 3.92 & 20.66 & 0001 & 0.68 & 1910 \\
\hline $\begin{array}{l}2 \\
2\end{array}$ & $\begin{array}{l}281 \\
282\end{array}$ & $\begin{array}{l}2.09 \\
4.28\end{array}$ & $\begin{array}{l}15.87 \\
22.15\end{array}$ & $\begin{array}{l}1310 \\
1551\end{array}$ & $\begin{array}{l}0.68 \\
0.68\end{array}$ & $\begin{array}{l}0838 \\
2057\end{array}$ \\
\hline 2 & 283 & 4.24 & 19.30 & 0105 & 0.68 & 2345 \\
\hline & $\begin{array}{l}284 \\
285\end{array}$ & $\begin{array}{l}2.65 \\
1.86\end{array}$ & $\begin{array}{l}15.06 \\
10.59\end{array}$ & $\begin{array}{l}1328 \\
1308\end{array}$ & $\begin{array}{l}0.68 \\
0.68\end{array}$ & $\begin{array}{l}2256 \\
0040\end{array}$ \\
\hline 2 & 286 & 0.81 & 3.34 & 0435 & 0.68 & 0401 \\
\hline 2 & 287 & 1.34 & 20.69 & 2230 & 0.68 & 0029 \\
\hline 2 & 288 & 3.69 & 22.14 & 1406 & 0.68 & 2347 \\
\hline 2 & 289 & 1.60 & 7.36 & 1214 & 0.68 & 0916 \\
\hline 2 & 290 & 2.44 & 10.06 & 0529 & 0.68 & 2305 \\
\hline 2 & 291 & 3.13 & 16.87 & 1429 & 0.68 & 2300 \\
\hline 2 & 292 & 3.94 & 19.36 & 1417 & 0.68 & 1953 \\
\hline 2 & 293 & 2.00 & 14.77 & 0039 & 0.68 & 0605 \\
\hline 2 & 294 & 1.72 & 9.82 & 2030 & 0.68 & 0152 \\
\hline 2 & 295 & 2.35 & 13.20 & 1252 & 0.68 & 0653 \\
\hline 2 & 296 & 4.53 & 34.83 & 1813 & 0.68 & 0357 \\
\hline 2 & 297 & 6.03 & 29.18 & 1845 & 0.68 & 2154 \\
\hline 2 & 298 & 2.80 & 15.09 & 1041 & 0.68 & 0404 \\
\hline
\end{tabular}


Table 3. --Summary of 1986 wind-speed data at the raft station--Continued

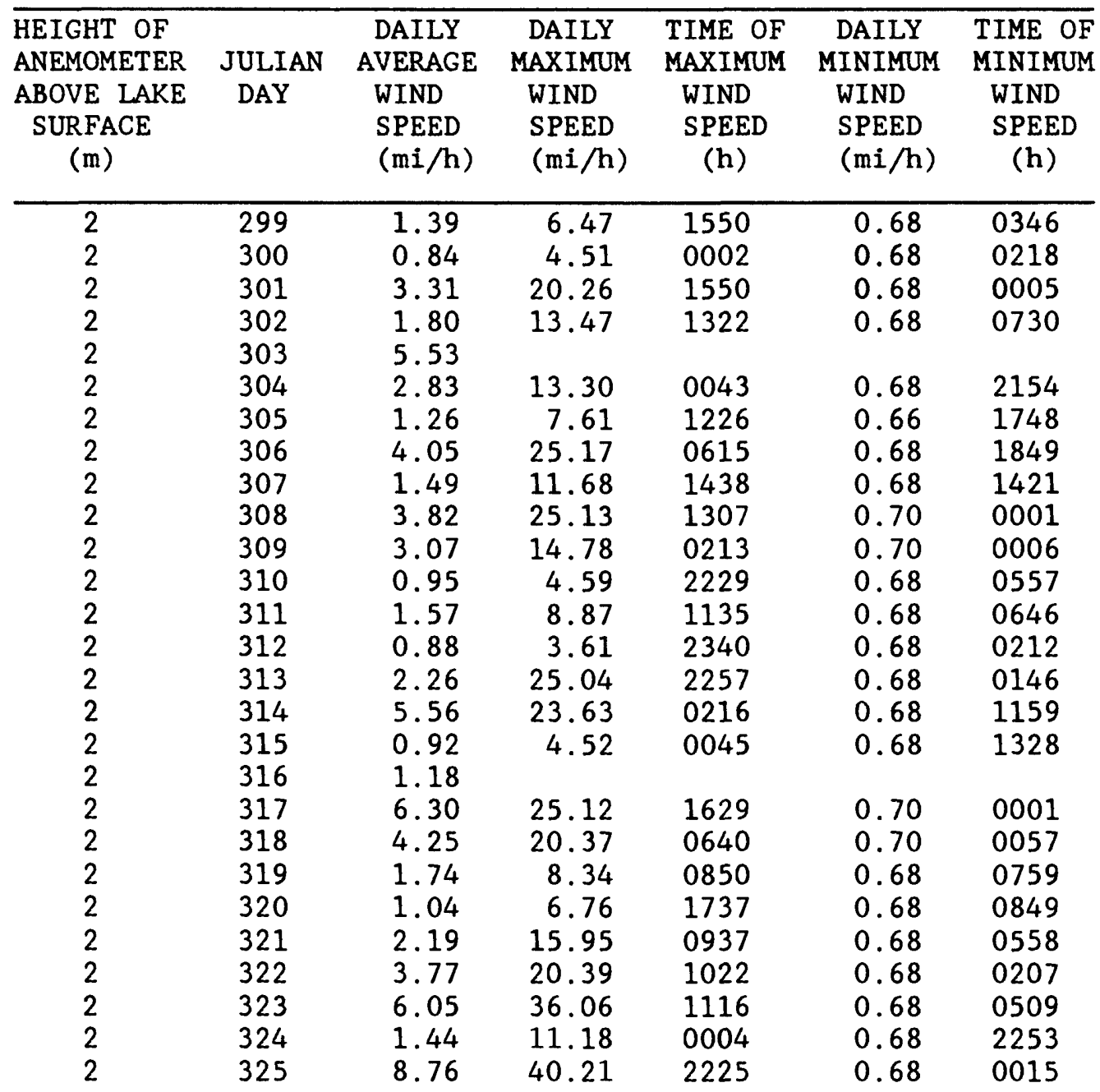


Table 3. --Summary of 1986 wind-speed data at the raft station--Continued

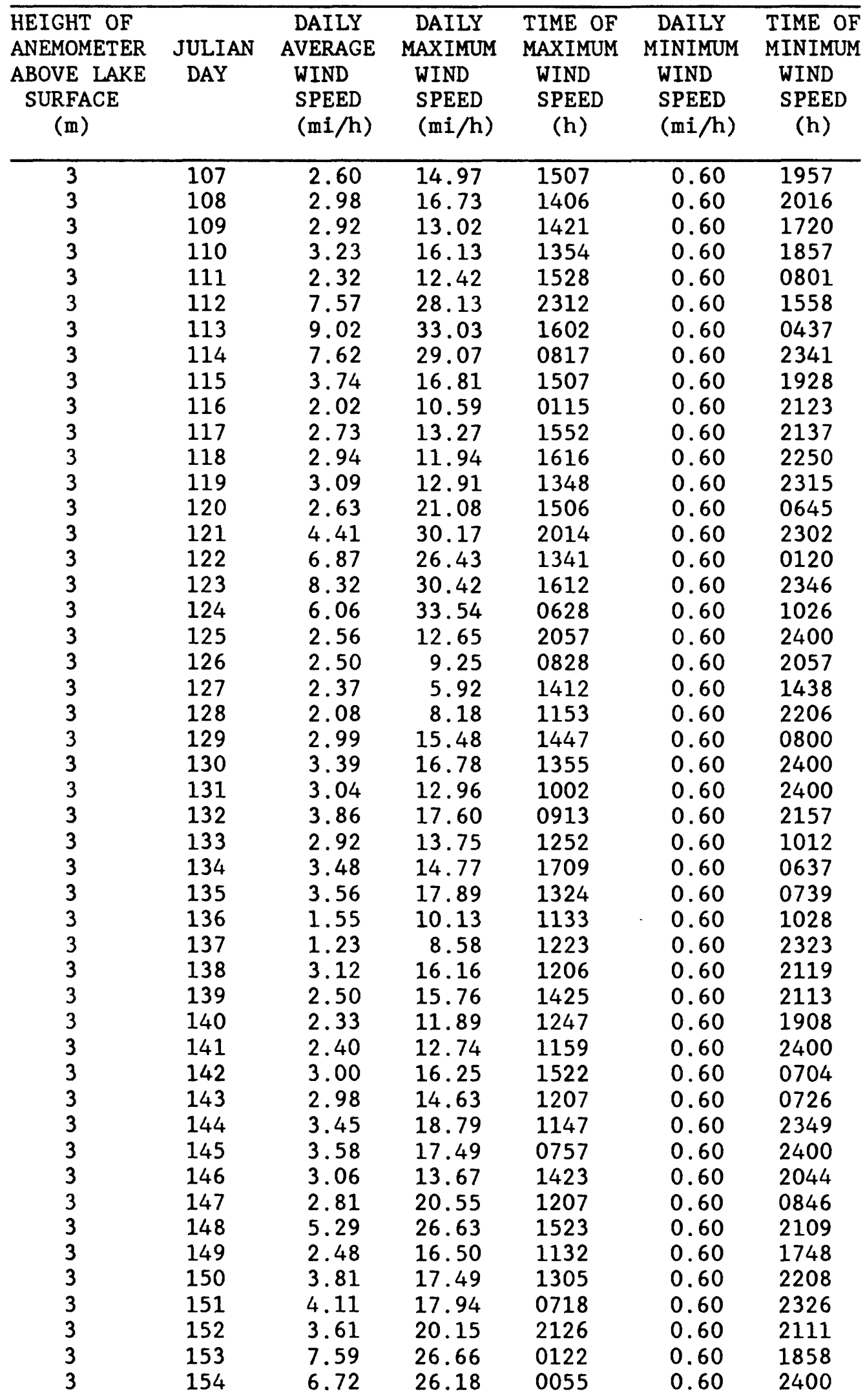


Table 3. --Summary of 1986 wind-speed data at the raft station--Continued

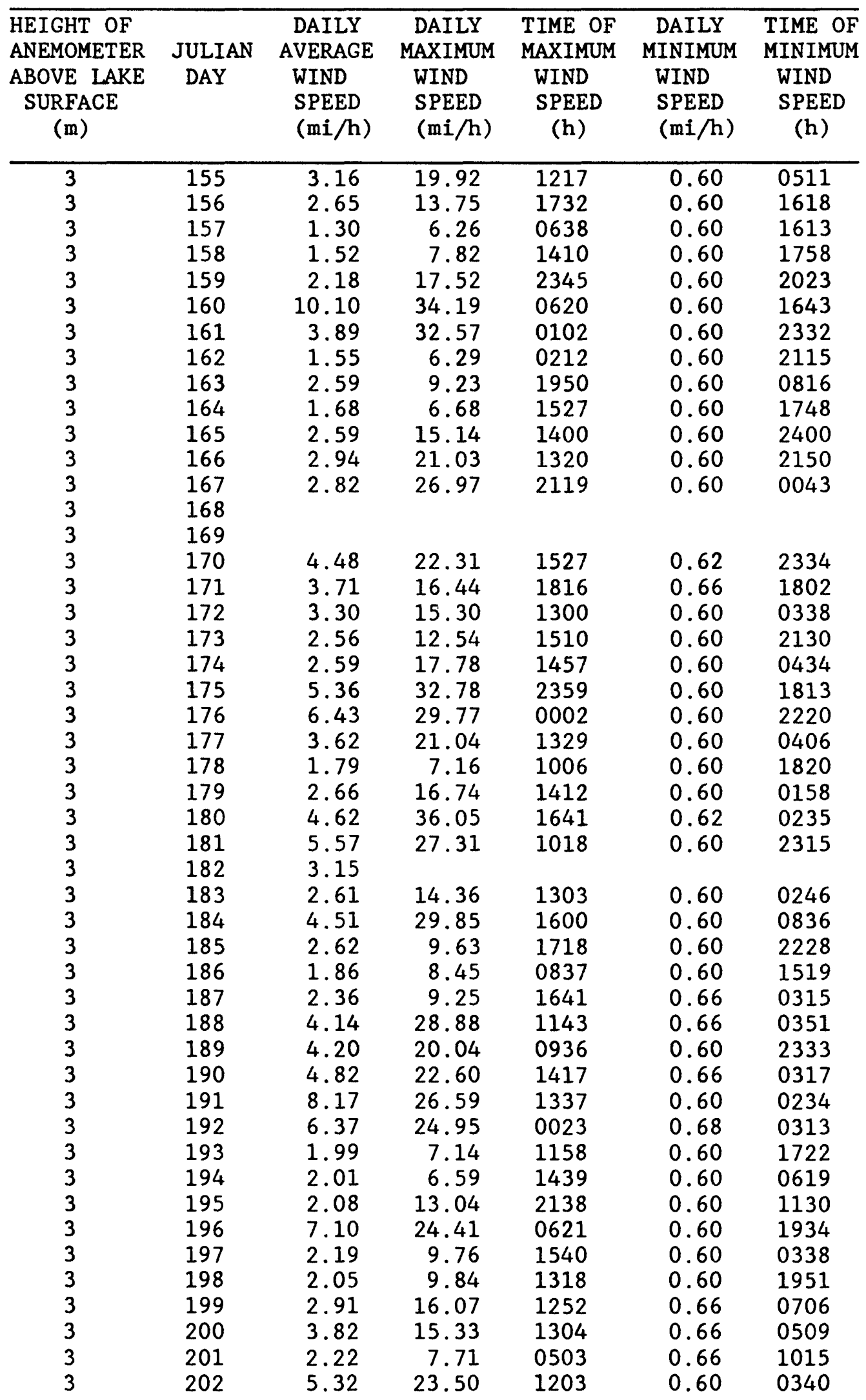


Table 3. --Summary of 1986 wind-speed data at the raft station--Continued

\begin{tabular}{|c|c|c|c|c|c|c|}
\hline $\begin{array}{l}\text { HEIGHT OF } \\
\text { ANEMOMETER } \\
\text { ABOVE LAKE } \\
\text { SURFACE } \\
\text { (m) }\end{array}$ & $\begin{array}{l}\text { JULIAN } \\
\text { DAY }\end{array}$ & $\begin{array}{l}\text { DAILY } \\
\text { AVERAGE } \\
\text { WIND } \\
\text { SPEED } \\
(\mathrm{mi} / \mathrm{h})\end{array}$ & $\begin{array}{l}\text { DAILY } \\
\text { MAXIMUM } \\
\text { WIND } \\
\text { SPEED } \\
(\mathrm{mi} / \mathrm{h})\end{array}$ & $\begin{array}{l}\text { TIME OF } \\
\text { MAXIMUM } \\
\text { WIND } \\
\text { SPEED } \\
(\mathrm{h})\end{array}$ & $\begin{array}{l}\text { DAILY } \\
\text { MINIMUM } \\
\text { WIND } \\
\text { SPEED } \\
(\mathrm{mi} / \mathrm{h})\end{array}$ & $\begin{array}{l}\text { TIME OF } \\
\text { MINIMUM } \\
\text { WIND } \\
\text { SPEED } \\
\text { (h) }\end{array}$ \\
\hline 3 & 203 & 2.74 & 11.10 & 1420 & 0.60 & 0313 \\
\hline 3 & 204 & 3.07 & 14.45 & 1151 & 0.64 & 1717 \\
\hline 3 & 205 & 2.98 & 15.38 & 1157 & 0.60 & 0342 \\
\hline 3 & 206 & 1.86 & 13.24 & 1554 & 0.60 & 1728 \\
\hline 3 & 207 & 2.33 & 21.40 & 1648 & 0.60 & 2059 \\
\hline 3 & 208 & 2.12 & 11.39 & 1349 & 0.68 & 0036 \\
\hline 3 & 209 & 2.94 & 13.09 & 1402 & 0.60 & 0433 \\
\hline 3 & 210 & 2.40 & & & & \\
\hline 3 & 211 & 2.56 & & & & \\
\hline 3 & 212 & 2.12 & 10.76 & 1330 & 0.60 & 1729 \\
\hline 3 & 213 & 1.66 & 8.99 & 2124 & 0.60 & 1255 \\
\hline 3 & 214 & 2.53 & 10.31 & 1759 & 0.60 & 0227 \\
\hline 3 & 215 & 2.73 & 13.76 & 1459 & 0.60 & 0326 \\
\hline 3 & 216 & 3.15 & & & & \\
\hline 3 & 217 & 3.06 & 13.71 & 1207 & 0.60 & 0455 \\
\hline 3 & 218 & 2.80 & 13.51 & & & \\
\hline 3 & 219 & 1.80 & 8.13 & 1937 & 0.60 & 2047 \\
\hline 3 & 220 & 2.64 & 17.70 & 1447 & 0.60 & 2212 \\
\hline 3 & 221 & 2.47 & 14.97 & 1348 & 0.60 & 2253 \\
\hline 3 & 222 & 2.83 & 14.90 & 0616 & 0.60 & 0346 \\
\hline 3 & 223 & 4.04 & 17.30 & 1154 & 0.60 & 0718 \\
\hline 3 & 224 & 3.60 & 17.57 & 0958 & 0.60 & 0313 \\
\hline 3 & 225 & 3.30 & 12.20 & 1304 & 0.66 & 2107 \\
\hline 3 & 226 & 2.23 & 14.36 & 1452 & 0.60 & 2355 \\
\hline 3 & 227 & 1.76 & 7.84 & 1305 & 0.60 & 1200 \\
\hline 3 & 228 & 1.49 & 7.36 & 1538 & 0.60 & 0307 \\
\hline 3 & 229 & 2.84 & 12.17 & 1155 & 0.66 & 0700 \\
\hline 3 & 230 & 2.27 & 10.52 & 1222 & 0.64 & 1436 \\
\hline 3 & 231 & 3.50 & 15.72 & 1552 & 0.60 & 2015 \\
\hline 3 & 232 & 2.76 & & & & \\
\hline 3 & 233 & 3.12 & 15.16 & 1521 & 0.60 & 0034 \\
\hline 3 & 234 & 5.47 & 23.97 & 1604 & 0.60 & 0402 \\
\hline 3 & 235 & 2.47 & 12.75 & 1023 & 0.60 & 1709 \\
\hline 3 & 236 & 6.87 & 25.20 & 2144 & 0.60 & 0423 \\
\hline 3 & 237 & 7.48 & 25.10 & 0247 & 0.60 & 0943 \\
\hline 3 & 238 & 2.69 & 15.26 & 1140 & 0.60 & 0108 \\
\hline 3 & 239 & 4.43 & 28.07 & 2322 & 0.60 & 0427 \\
\hline 3 & 240 & 5.74 & 26.40 & 0019 & 0.60 & 2331 \\
\hline 3 & 241 & 4.40 & 19.91 & 0137 & 0.60 & 0547 \\
\hline 3 & 242 & 3.45 & 16.82 & 1029 & 0.60 & 0524 \\
\hline 3 & 243 & 3.73 & 17.23 & 1032 & 0.60 & 2320 \\
\hline 3 & 244 & 2.14 & 8.11 & 1250 & 0.60 & 0625 \\
\hline 3 & 245 & 1.97 & & & & \\
\hline 3 & 246 & 2.88 & & & & \\
\hline 3 & 247 & 2.62 & 8.70 & 1204 & 0.60 & 0201 \\
\hline 3 & 248 & 1.43 & 6.56 & 1209 & 0.60 & 1515 \\
\hline 3 & 249 & 3.28 & 14.73 & 1303 & 0.60 & 0521 \\
\hline 3 & 250 & 2.93 & 11.11 & 1423 & 0.60 & 0104 \\
\hline
\end{tabular}


Table 3. --Summary of 1986 wind-speed data at the raft station--Continued

\begin{tabular}{|c|c|c|c|c|c|c|}
\hline $\begin{array}{l}\text { HEIGHT OF } \\
\text { ANEMOMETER } \\
\text { ABOVE LAKE } \\
\text { SURFACE } \\
\text { (m) }\end{array}$ & $\begin{array}{l}\text { JULIAN } \\
\text { DAY }\end{array}$ & $\begin{array}{l}\text { DAILY } \\
\text { AVERAGE } \\
\text { WIND } \\
\text { SPEED } \\
(\mathrm{mi} / \mathrm{h})\end{array}$ & $\begin{array}{l}\text { DAILY } \\
\text { MAXIMUM } \\
\text { WIND } \\
\text { SPEED } \\
(\mathrm{mi} / \mathrm{h})\end{array}$ & $\begin{array}{l}\text { TIME OF } \\
\text { MAXIMUM } \\
\text { WIND } \\
\text { SPEED } \\
\text { (h) }\end{array}$ & $\begin{array}{l}\text { DAILY } \\
\text { MINIMUM } \\
\text { WIND } \\
\text { SPEED } \\
(\mathrm{mi} / \mathrm{h})\end{array}$ & $\begin{array}{l}\text { TIME OF } \\
\text { MINIMUM } \\
\text { WIND } \\
\text { SPEED } \\
\text { (h) }\end{array}$ \\
\hline $\begin{array}{l}3 \\
3 \\
3 \\
3 \\
3 \\
3 \\
3 \\
3 \\
3 \\
3 \\
3 \\
3 \\
3 \\
3 \\
3 \\
3 \\
3 \\
3 \\
3 \\
3 \\
3 \\
3 \\
3 \\
3\end{array}$ & $\begin{array}{l}251 \\
252 \\
253 \\
254 \\
255 \\
256 \\
257 \\
258 \\
259 \\
260 \\
261 \\
262 \\
263 \\
264 \\
265 \\
266 \\
267 \\
268 \\
269 \\
270 \\
271 \\
272 \\
273 \\
274\end{array}$ & $\begin{array}{l}3.32 \\
2.78 \\
2.13 \\
1.15 \\
3.08 \\
5.46 \\
6.43 \\
3.79 \\
7.64 \\
6.11 \\
2.58 \\
2.80 \\
1.37 \\
2.06 \\
2.11 \\
1.53 \\
2.56 \\
4.78 \\
3.09 \\
3.26 \\
1.66 \\
1.43 \\
3.06 \\
5.10\end{array}$ & $\begin{array}{r}15.24 \\
14.92 \\
11.00 \\
5.31 \\
20.80 \\
26.64 \\
26.08 \\
18.95 \\
27.86 \\
23.83 \\
11.67 \\
20.90 \\
5.39 \\
6.81 \\
12.36 \\
7.76 \\
15.35 \\
27.75 \\
20.66 \\
11.62 \\
8.54 \\
7.65 \\
27.62 \\
23.62\end{array}$ & $\begin{array}{l}1019 \\
1134 \\
1154 \\
1346 \\
1531 \\
2138 \\
1023 \\
1430 \\
0956 \\
0813 \\
1207 \\
1301 \\
0613 \\
1610 \\
1035 \\
1826 \\
1253 \\
0918 \\
2124 \\
0357 \\
1338 \\
1258 \\
1352 \\
1003\end{array}$ & $\begin{array}{l}0.60 \\
0.60 \\
0.60 \\
0.60 \\
0.60 \\
0.68 \\
0.60 \\
0.60 \\
0.60 \\
0.60 \\
0.60 \\
0.60 \\
0.60 \\
0.60 \\
0.60 \\
0.60 \\
0.60 \\
0.60 \\
0.60 \\
0.60 \\
0.60 \\
0.60 \\
0.68 \\
0.62\end{array}$ & $\begin{array}{l}2059 \\
0519 \\
0322 \\
0215 \\
0634 \\
0628 \\
1947 \\
0653 \\
2108 \\
1906 \\
0625 \\
1856 \\
0019 \\
0557 \\
1024 \\
2116 \\
0145 \\
0138 \\
0312 \\
0804 \\
0208 \\
0958 \\
0202 \\
0319\end{array}$ \\
\hline 3 & 275 & 2.08 & & & & \\
\hline & $\begin{array}{l}276 \\
277\end{array}$ & $\begin{array}{l}2.46 \\
3.90\end{array}$ & $\begin{array}{l}17.97 \\
19.20\end{array}$ & $\begin{array}{l}1204 \\
0942\end{array}$ & $\begin{array}{l}0.60 \\
0.60\end{array}$ & $\begin{array}{l}1503 \\
0136\end{array}$ \\
\hline 3 & 278 & 5.09 & 21.42 & 1123 & 0.60 & 2317 \\
\hline 3 & 279 & 7.94 & 37.10 & 2105 & 0.60 & 0604 \\
\hline 3 & 280 & 4.40 & 21.00 & 0001 & 0.60 & 2146 \\
\hline $\begin{array}{l}3 \\
3\end{array}$ & $\begin{array}{l}281 \\
282\end{array}$ & $\begin{array}{l}2.49 \\
4.69\end{array}$ & $\begin{array}{l}16.12 \\
21.13\end{array}$ & $\begin{array}{l}1312 \\
1551\end{array}$ & $\begin{array}{l}0.60 \\
0.60\end{array}$ & $\begin{array}{l}0215 \\
1008\end{array}$ \\
\hline & $\begin{array}{l}283 \\
284\end{array}$ & $\begin{array}{l}4.65 \\
2.96\end{array}$ & $\begin{array}{l}21.56 \\
14.37\end{array}$ & $\begin{array}{l}0105 \\
1328\end{array}$ & $\begin{array}{l}0.60 \\
0.60\end{array}$ & $\begin{array}{l}1854 \\
2235\end{array}$ \\
\hline & $\begin{array}{l}285 \\
286\end{array}$ & $\begin{array}{l}2.17 \\
0.91\end{array}$ & $\begin{array}{r}10.32 \\
3.68\end{array}$ & $\begin{array}{l}1123 \\
0129\end{array}$ & $\begin{array}{l}0.60 \\
0.60\end{array}$ & $\begin{array}{l}2331 \\
0712\end{array}$ \\
\hline 3 & 287 & 1.51 & 20.73 & 2251 & 0.60 & 0632 \\
\hline 3 & 288 & 3.95 & 23.22 & 1406 & 0.60 & 0715 \\
\hline 3 & 289 & 1.83 & 7.63 & 1228 & 0.60 & 0711 \\
\hline 3 & 290 & 2.70 & 11.00 & 0529 & 0.60 & 0155 \\
\hline 3 & 291 & 3.38 & 17.93 & 1429 & 0.60 & 2138 \\
\hline 3 & 292 & 4.31 & 19.83 & 1515 & 0.60 & 1021 \\
\hline 3 & 293 & 2.25 & 16.34 & 0039 & 0.60 & 0239 \\
\hline 3 & 294 & 2.02 & 11.38 & 2030 & 0.60 & 1300 \\
\hline 3 & 295 & 2.74 & 14.65 & 0052 & 0.60 & 0623 \\
\hline 3 & 296 & 5.09 & 34.59 & 1813 & 0.60 & 0225 \\
\hline 3 & 297 & 6.79 & 30.33 & 1845 & 0.60 & 0121 \\
\hline 3 & 298 & 3.39 & 15.46 & 0058 & 0.60 & 0452 \\
\hline
\end{tabular}


Table 3. --Summary of 1986 wind-speed data at the raft station-Continued

\begin{tabular}{|c|c|c|c|c|c|c|}
\hline $\begin{array}{l}\text { HEIGHT OF } \\
\text { ANEMOMETER } \\
\text { ABOVE LAKE } \\
\text { SURFACE } \\
\text { (m) }\end{array}$ & $\begin{array}{l}\text { JULIAN } \\
\text { DAY }\end{array}$ & $\begin{array}{l}\text { DAILY } \\
\text { AVERAGE } \\
\text { WIND } \\
\text { SPEED } \\
(\mathrm{mi} / \mathrm{h})\end{array}$ & $\begin{array}{l}\text { DAILY } \\
\text { MAXIMUM } \\
\text { WIND } \\
\text { SPEED } \\
(\mathrm{mi} / \mathrm{h})\end{array}$ & $\begin{array}{l}\text { TIME OF } \\
\text { MAXIMUM } \\
\text { WIND } \\
\text { SPEED } \\
\text { (h) }\end{array}$ & $\begin{array}{l}\text { DAILY } \\
\text { MINIMUM } \\
\text { WIND } \\
\text { SPEED } \\
(\mathrm{mi} / \mathrm{h})\end{array}$ & $\begin{array}{l}\text { TIME OF } \\
\text { MINIMUM } \\
\text { WIND } \\
\text { SPEED } \\
\text { (h) }\end{array}$ \\
\hline $\begin{array}{l}3 \\
3 \\
3 \\
3 \\
3 \\
3 \\
3 \\
3 \\
3 \\
3 \\
3 \\
3 \\
3 \\
3 \\
3 \\
3 \\
3 \\
3 \\
3 \\
3 \\
3 \\
3 \\
3 \\
3 \\
3 \\
3 \\
3\end{array}$ & $\begin{array}{l}299 \\
300 \\
301 \\
302 \\
303 \\
304 \\
305 \\
306 \\
307 \\
308 \\
309 \\
310 \\
311 \\
312 \\
313 \\
314 \\
315 \\
316 \\
317 \\
318 \\
319 \\
320 \\
321 \\
322 \\
323 \\
324 \\
325\end{array}$ & $\begin{array}{l}1.89 \\
1.04 \\
3.76 \\
2.21 \\
6.13 \\
3.38 \\
1.63 \\
4.44 \\
1.76 \\
4.09 \\
3.55 \\
1.14 \\
2.05 \\
1.20 \\
2.61 \\
6.11 \\
1.40 \\
1.42 \\
6.98 \\
5.03 \\
2.12 \\
1.38 \\
2.44 \\
4.14 \\
6.45 \\
1.82 \\
9.33\end{array}$ & $\begin{array}{r}7.03 \\
4.47 \\
22.70 \\
13.79 \\
14.11 \\
7.66 \\
24.85 \\
11.96 \\
24.65 \\
17.10 \\
4.86 \\
8.91 \\
4.09 \\
25.23 \\
23.86 \\
4.69\end{array}$ & $\begin{array}{l}1550 \\
0123 \\
1550 \\
1325 \\
0043 \\
1035 \\
0615 \\
1438 \\
1307 \\
0213 \\
2229 \\
1135 \\
2332 \\
2257 \\
0048 \\
0223\end{array}$ & $\begin{array}{l}0.60 \\
0.60 \\
0.66 \\
0.60 \\
0.60 \\
0.60 \\
0.60 \\
0.60 \\
0.60 \\
0.60 \\
0.60 \\
0.60 \\
0.60 \\
0.60 \\
0.60 \\
0.60\end{array}$ & $\begin{array}{l}0535 \\
0351 \\
1953 \\
0517 \\
0621 \\
2355 \\
1829 \\
1714 \\
0519 \\
2325 \\
1807 \\
0215 \\
2014 \\
0523 \\
1751 \\
2225\end{array}$ \\
\hline
\end{tabular}




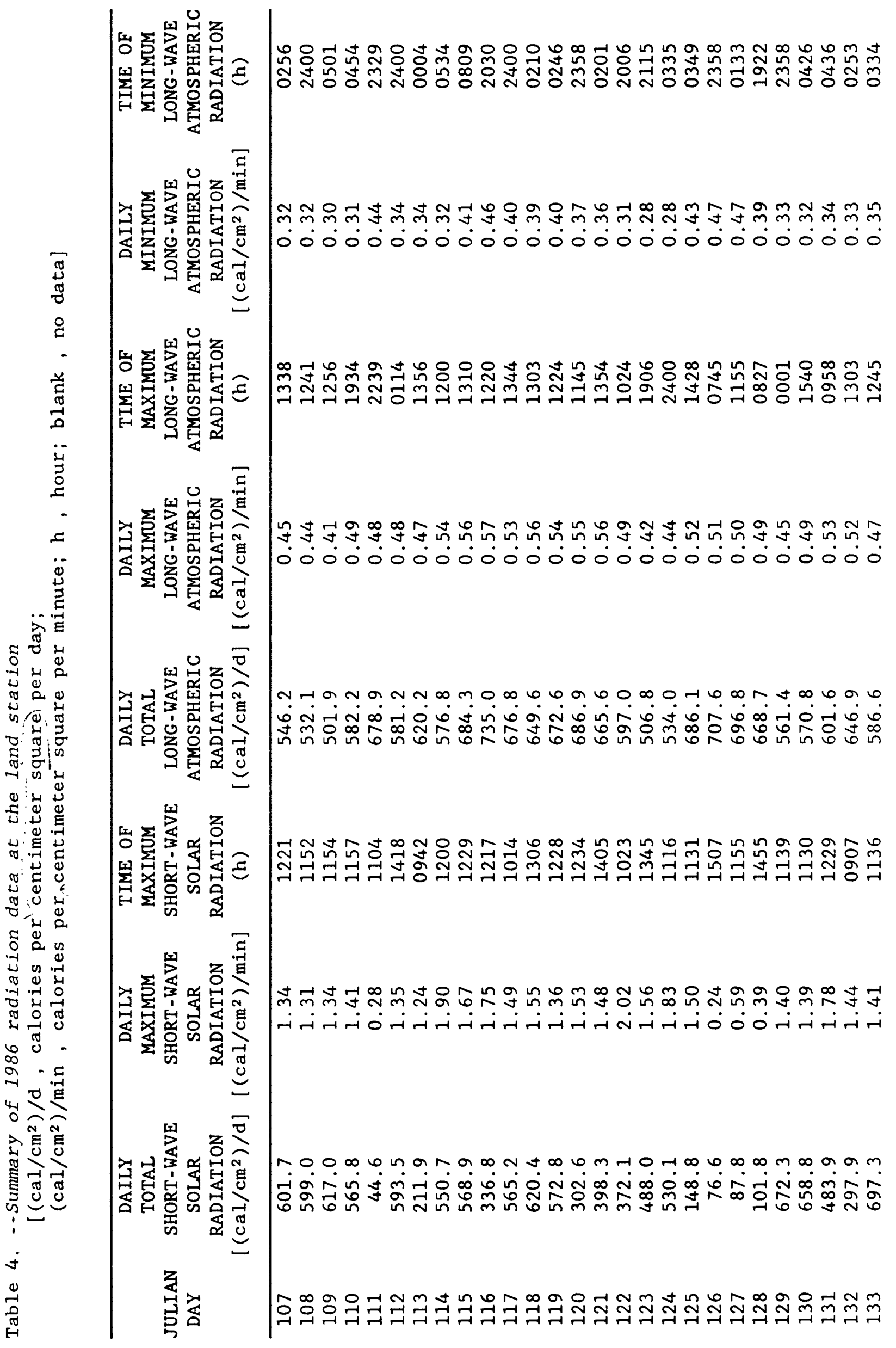




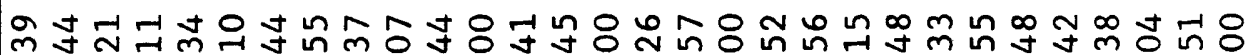
莳宩

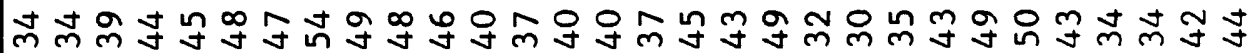
$\dot{0} \dot{0} \dot{0} \dot{0} 0 \dot{0} \dot{0} 0 \dot{0} 0 \dot{0} 0 \dot{0} \dot{0} 0 \dot{0} 0 \dot{0} 0 \dot{0} 0 \dot{0}$

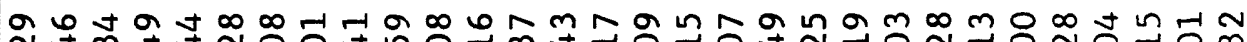
సิ

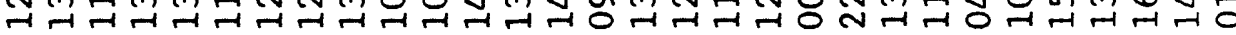

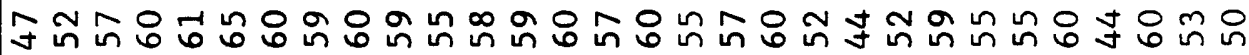

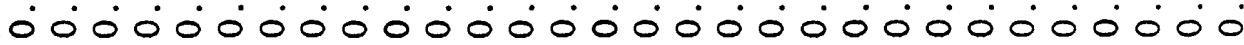

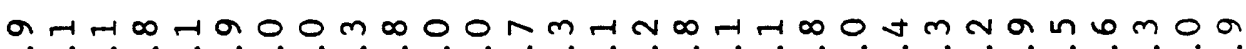

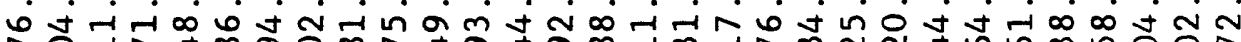

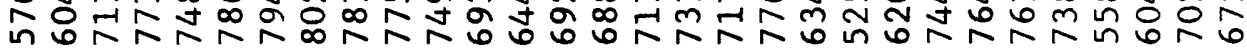

우의 연

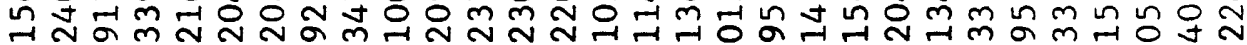

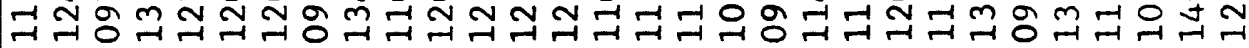

오웅 (1).

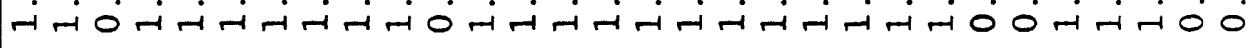

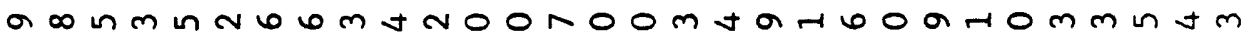

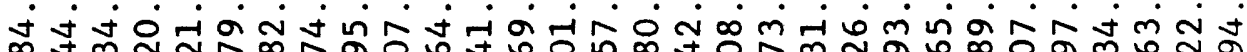

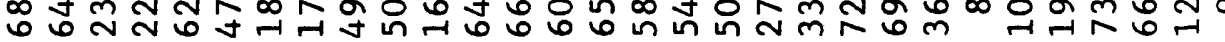

స్

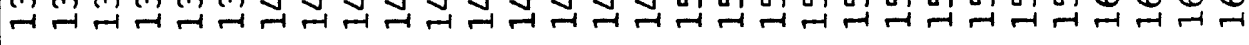




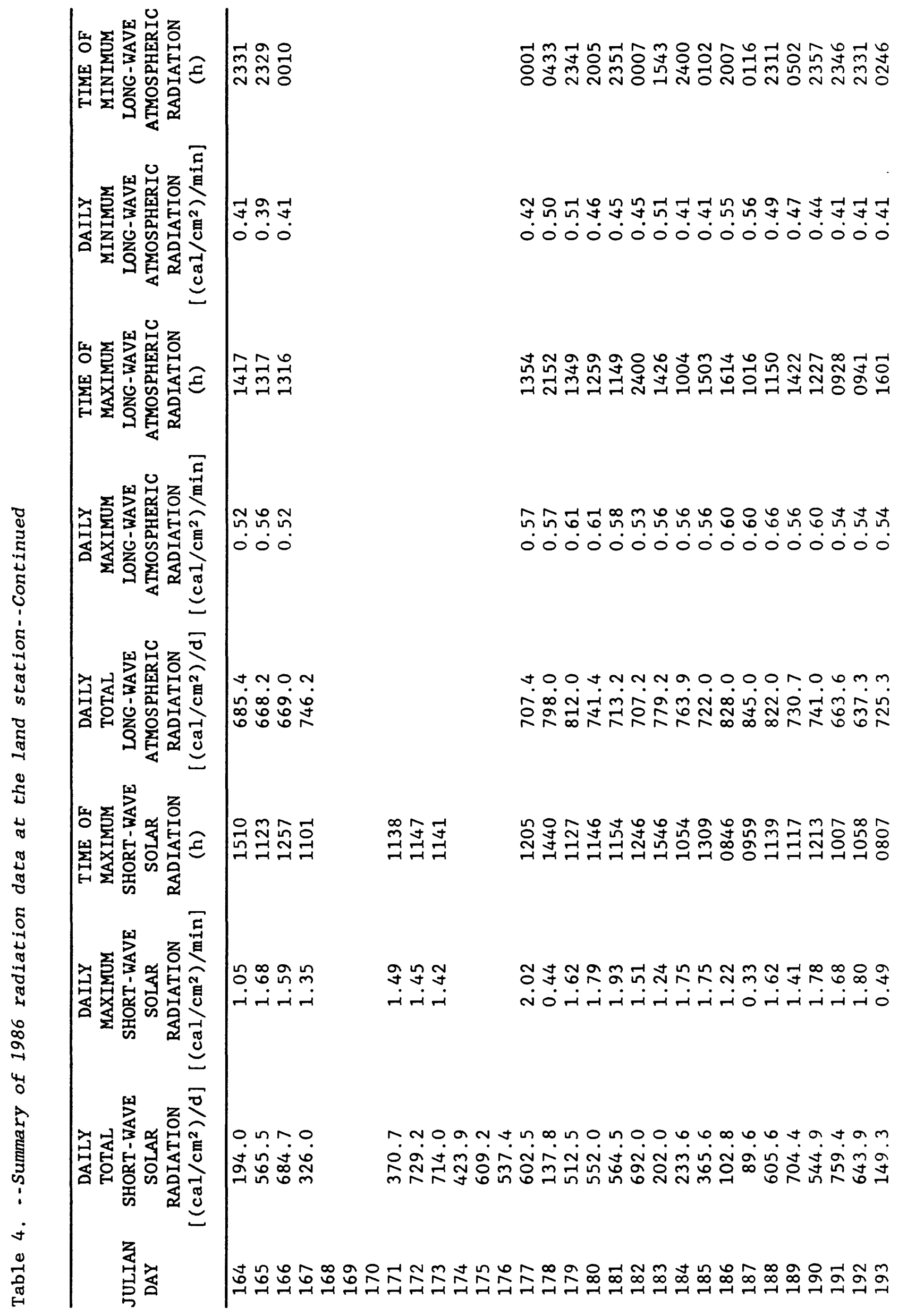




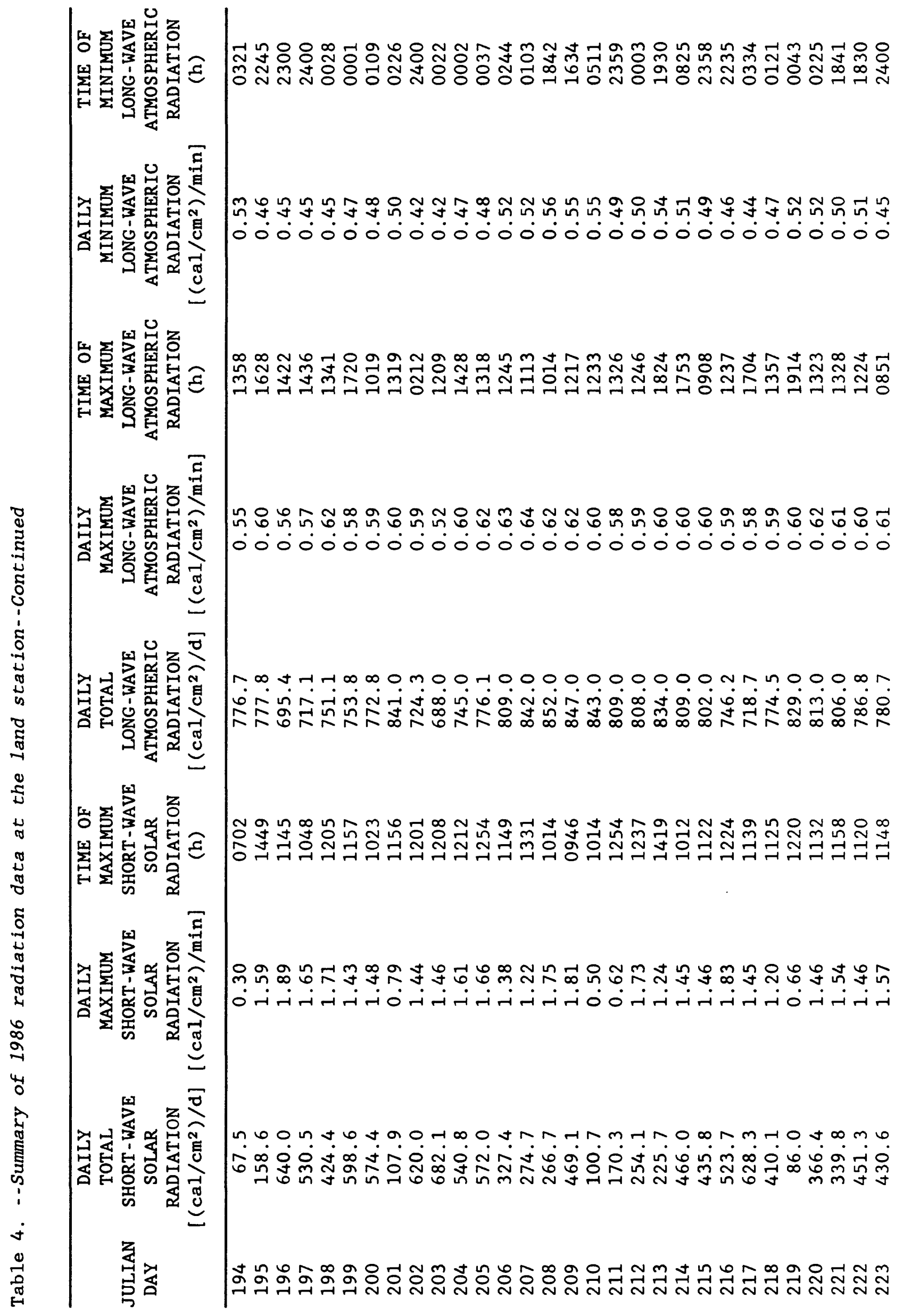




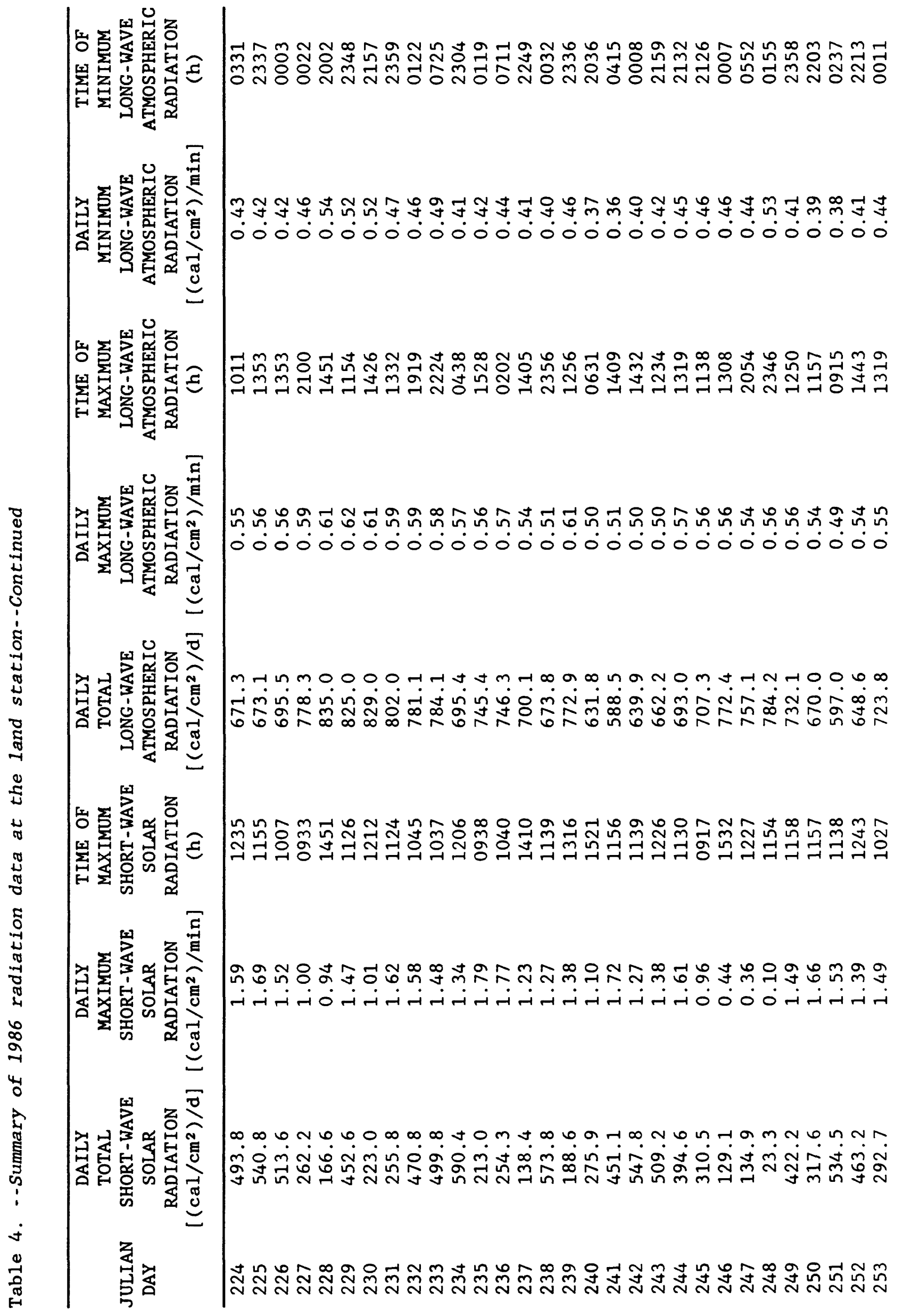




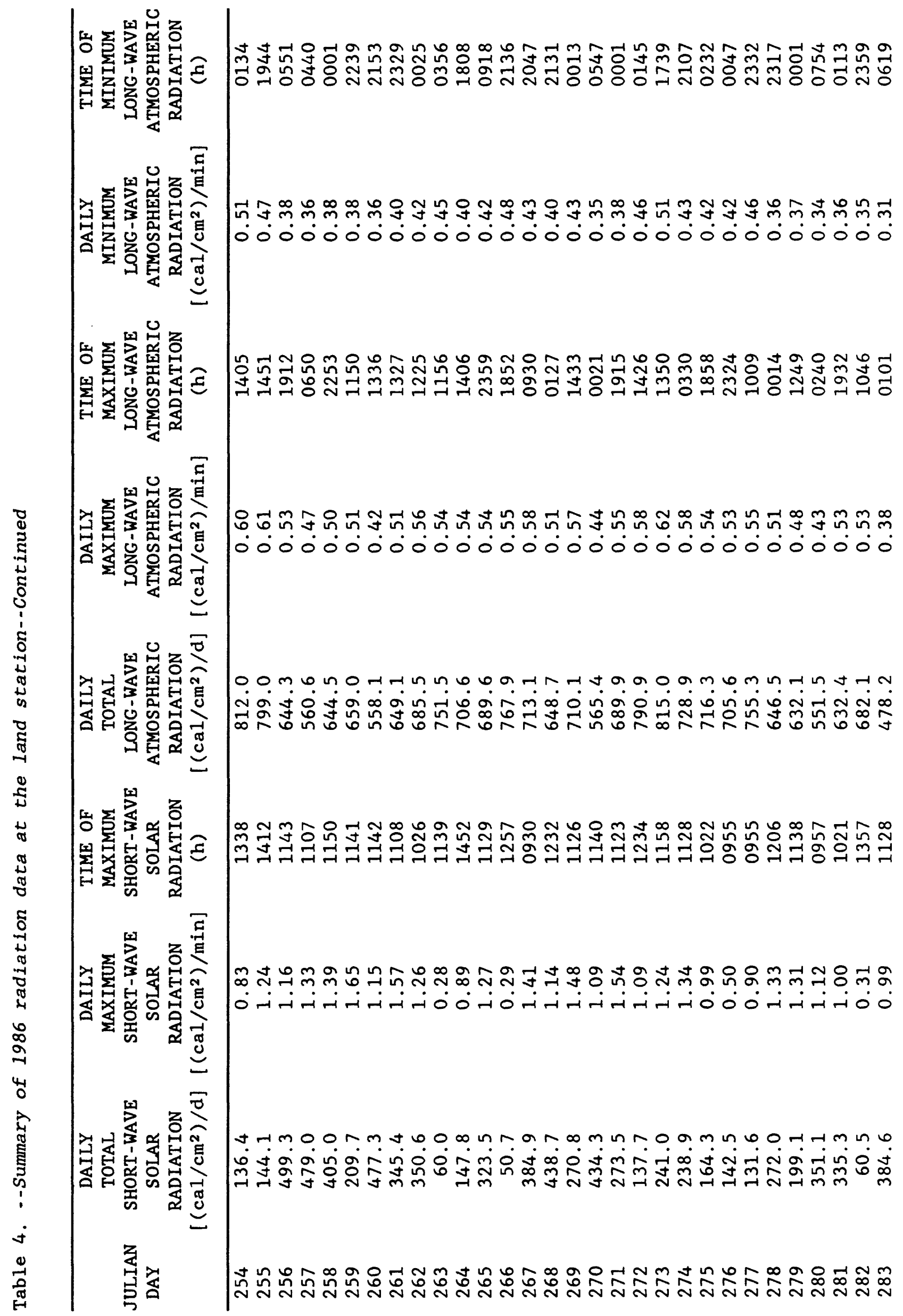




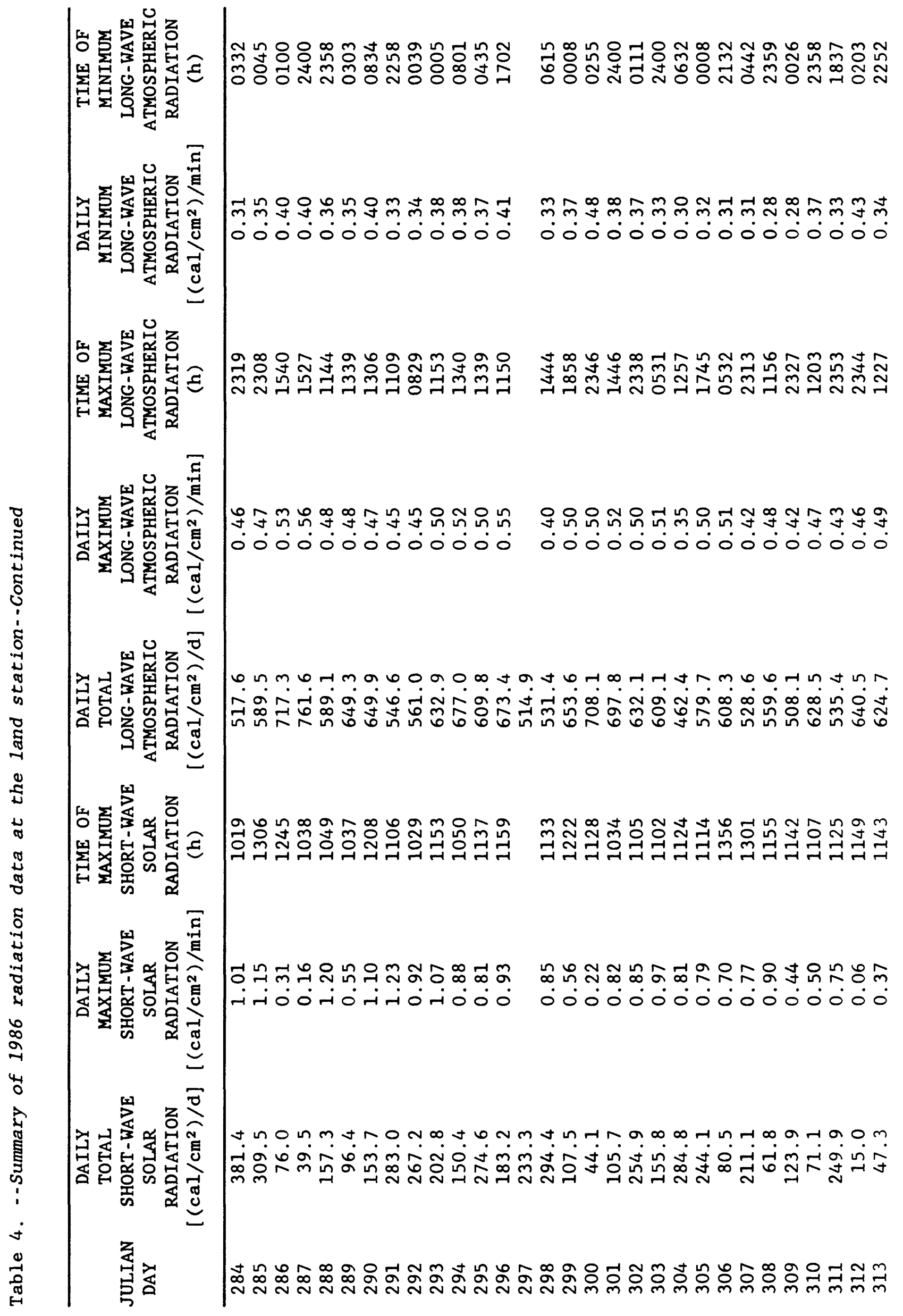




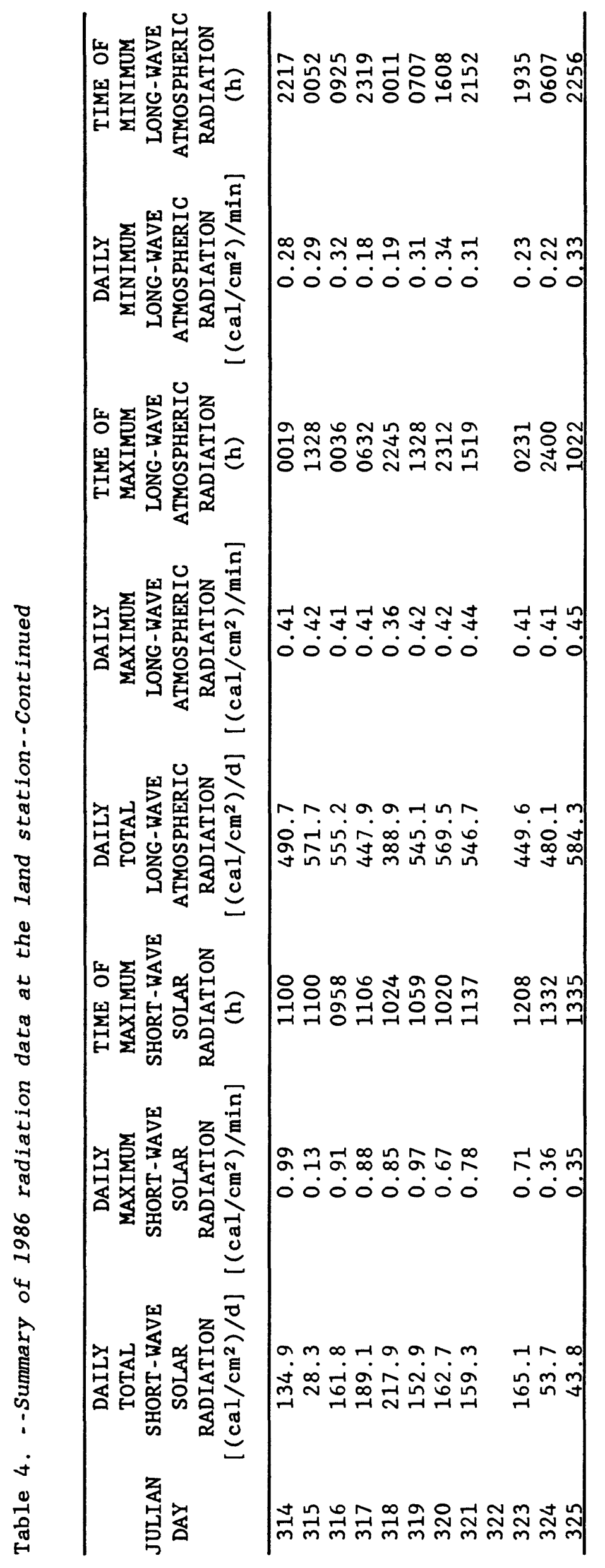

